University of Louisville

ThinkIR: The University of Louisville's Institutional Repository

$5-2017$

\title{
Trunk and respiratory motor control in typically developing children and its implications in children with chronic spinal cord injury.
}

\author{
Goutam Singh \\ University of Louisville
}

Follow this and additional works at: https://ir.library.louisville.edu/etd

Part of the Circulatory and Respiratory Physiology Commons, Kinesiotherapy Commons, Musculoskeletal, Neural, and Ocular Physiology Commons, Nervous System Diseases Commons, Neurosciences Commons, Physical Therapy Commons, Physiotherapy Commons, and the Respiratory Therapy Commons

\section{Recommended Citation}

Singh, Goutam, "Trunk and respiratory motor control in typically developing children and its implications in children with chronic spinal cord injury." (2017). Electronic Theses and Dissertations. Paper 2655. https://doi.org/10.18297/etd/2655

This Doctoral Dissertation is brought to you for free and open access by ThinkIR: The University of Louisville's Institutional Repository. It has been accepted for inclusion in Electronic Theses and Dissertations by an authorized administrator of ThinkIR: The University of Louisville's Institutional Repository. This title appears here courtesy of the author, who has retained all other copyrights. For more information, please contact thinkir@louisville.edu. 


\title{
TRUNK AND RESPIRATORY MOTOR CONTROL IN TYPICALLY DEVELOPING CHILDREN AND ITS IMPLICATIONS IN CHILDREN WITH CHRONIC SPINAL CORD INJURY
}

\author{
By \\ Goutam Singh, PT, MS. \\ B.S, KIPT, Belgaum, 2008 \\ M.S, University of Louisville, 2012

\begin{abstract}
A Dissertation
Submitted to the Faculty of in Partial Fulfillment of the Requirements for the Degree of Doctor of Philosophy in Physiology and Biophysics

Department of Physiology and Biophysics University of Louisville Louisville, KY
\end{abstract} \\ the School of Medicine, University of Louisville
}

May 2017 

TRUNK AND RESPIRATORY MOTOR CONTROL IN TYPICALLY

DEVELOPING CHILDREN AND ITS IMPLICATIONS IN CHILDREN WITH

CHRONIC SPINAL CORD INJURY

By

Goutam Singh, PT, MS.

B.S, KIPT, Belgaum, 2008

M.S, University of Louisville, 2012

A Dissertation ApSLRYHGon

February $21^{\text {st }} 2017$

By the following dissertation committee:

Alexander Ovechkin, MD., PhD

Andrea Behrman, PT., PhD

Irving Joshua, $\mathrm{PhD}$

Claudio Maldonado, $\mathrm{PhD}$

Dena Howland, PhD

David Lominadze, PhD

Daniela Terson de Paleville, PhD 


\section{DEDICATION}

This dissertation is dedicated to my parents, Late. Darshana Devi and Kuldeep

Singh, who taught me the value of hard work, time and education. 


\section{ACKNOWLEDGEMENTS}

I would like to express my sincere gratitude to my mentor Dr. Alexander Ovechkin, for his continuous support during graduate education, for his patience, motivation, enthusiasm, and immense knowledge. I am highly indebted and very grateful to Dr. Andrea Behrman for providing me an opportunity to be part of the NeuroRecovery Network and for suggesting work with the pediatric population. Besides my advisors, I would like to thank the rest of my dissertation committee: Dr. Irving G. Joshua, Dr. Dena R. Howland, Dr. Daniela Terson Depaleville, Dr. David Lominadze, and Dr. Claudio Maldonado for their insightful comments, encouragement, and challenging questions, which inspired me to broaden my research from various perspectives. I am also very grateful to Dr. Sevda Aslan for her continuous support, motivation, and guidance through this entire process. I am also thankful to my fellow lab mates, for the stimulating discussions, data collection and for all the fun we have had in the last four and half years. I also would like to thank my friends for being there to listen and motive me. Last but not the least; I would like to thank my family: my parents and my sister for their patience and constant support. 


\title{
ABSTRACT \\ TRUNK AND RESPIRATORY MOTOR CONTROL IN TYPICALLY DEVELOPING CHILDREN AND ITS IMPLICATION IN CHILDREN WITH SPINAL CORD INJURY
}

\author{
Goutam Singh
}

February 22017

\begin{abstract}
Independent sitting is a major milestone and is also a prerequisite for optimal performance of activities of daily living (ADLs). Development of sitting posture control is a dynamic process involving control of degrees of freedom of head and trunk. Traditionally, trunk has been modeled as a single unit (segment). However, recent studies have suggested that it is made up multiple spinal units, controlled by a combination of trunk muscles. During typical development, posture control of trunk is different for different trunk segments. This motor development of trunk control is a complex process due to constant interaction between the nervous system and environment. Any interruption in the normal processes would further complicate it, affecting the typical development of the child.
\end{abstract}

Poor trunk control and respiratory complications are characteristic features among children, adolescents, and adults with neuromuscular disorders. 
Pediatric spinal cord injuries (SCls) also pose a unique challenge compared to $\mathrm{SCl}$ in adults because of the continuous physical and cognitive development. Children with $\mathrm{SCl}$ exhibit deficits in trunk motor control, which impair their ability to sit or ambulate. These motor deficits can lead to compensatory changes in other segments of the body, which cause further deviations from typical postures. Trunk muscles have the dual function of supporting both, breathing and trunk posture. Therefore, in children with SCI, impairment of posture control will also affect respiratory functions. Depending on level and severity of injury, can potentially lead to severe respiratory insufficiency. Symptoms of respiratory insufficiency are highly correlated with the level and severity of spinal lesions. Injury at higher cervical and thoracic cord levels causes paresis and paralysis of most of the respiratory muscles, which increase the workload of breathing. As a result, respiratory complications are the leading cause of death among children with SCl. Therefore, impairment of posture control following SCl also affects respiratory functions.

Lack of appropriate tools to evaluate trunk motor control following SCI restricts the ability to understand its development and therefore it is a challenge to design treatments and strategies to slow down or prevent the progress of longterm effects of $\mathrm{SCl}$ in children. In this dissertation, we studied the postural control using a Segmental Assessment Trunk Control (SATCo) test and respiratory motor control using Respiratory Motor Control Assessment (RMCA) protocol in typically developing (TD) children and compared their results to age-matched children with SCI. 
Chapter I describes the background information about the trunk and respiratory motor control and how the injury to spinal cord impacts these motor functions. Chapter II illustrates the specific aims and hypothesis of this dissertation. Methods and protocols used to measure trunk and respiratory motor control in both, TD children and children with SCl are described in chapter III. Chapter IV and V describes the development of trunk and respiratory motor control in TD children.

Chapter VI and VII includes assessment of trunk and respiratory motor control in children with SCI, respectively. Chapter VIII describes the scientific findings and conclusions of this study including recommendations for future studies in this area. 


\section{TABLE OF CONTENTS}

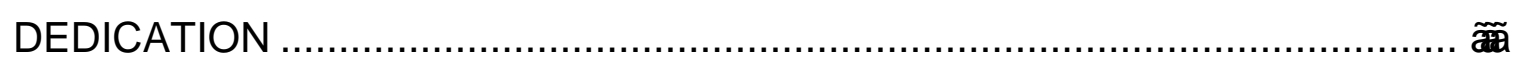

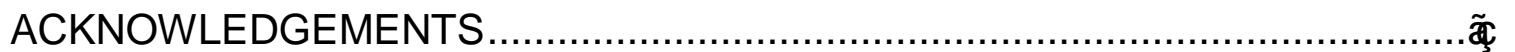

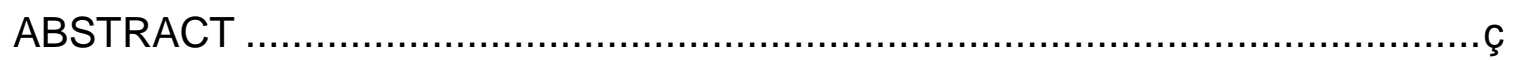

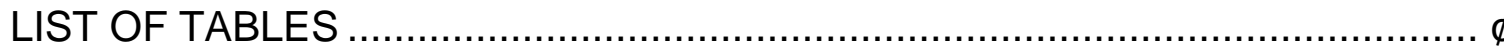

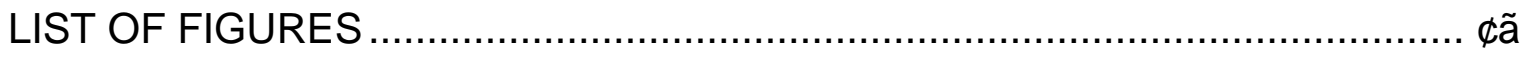

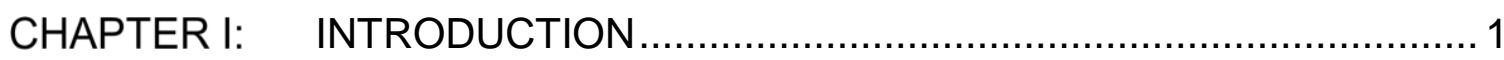

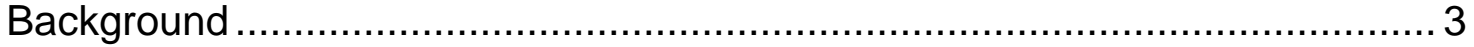

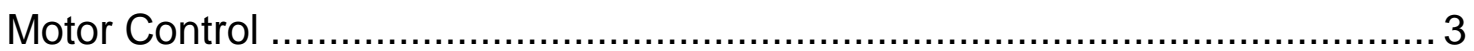

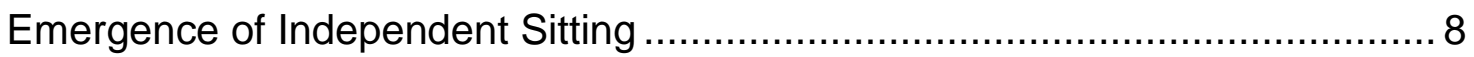

Respiratory Motor Control in Typically Developing Children .......................... 14

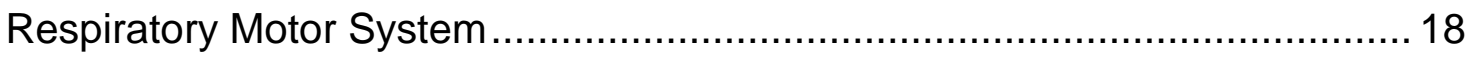

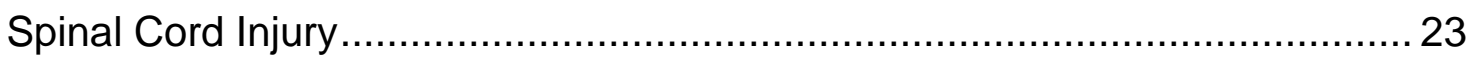

Development of Trunk Motor Control in Children after $\mathrm{SCl}$...........................25

Respiratory Motor Control in Children with $\mathrm{SCl}$......................................... 27

CHAPTER II: HYPOTHESIS AND SPECIFIC AIMS .................................... 32

CHAPTER III: METHODS AND MATERIAL ................................................ 34

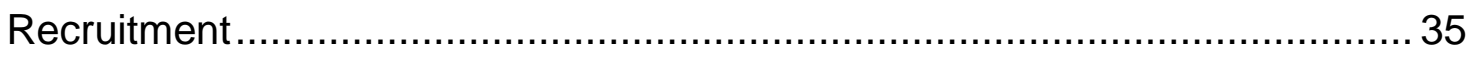

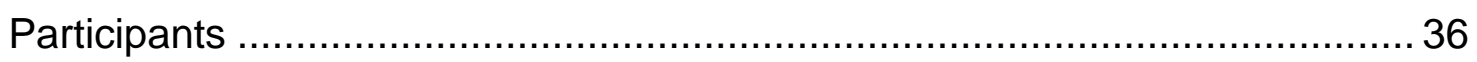

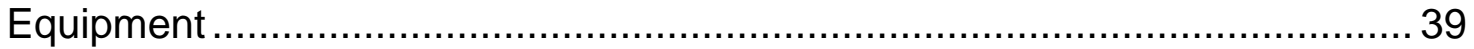

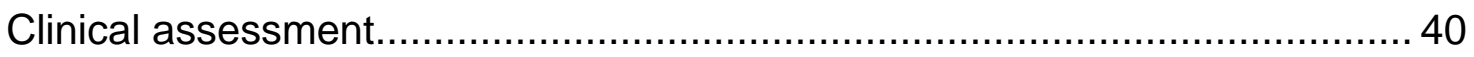

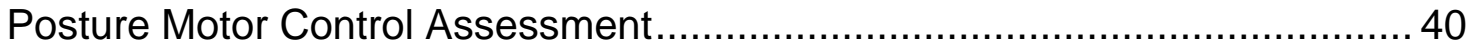

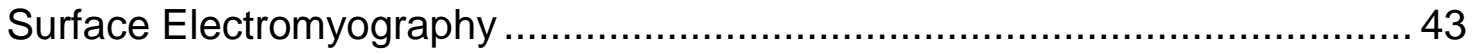

Respiratory Motor Control Assessment....................................................... 45

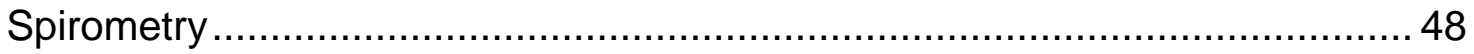

Maximum Expiratory and Inspiratory Airway Pressure ….............................. 50

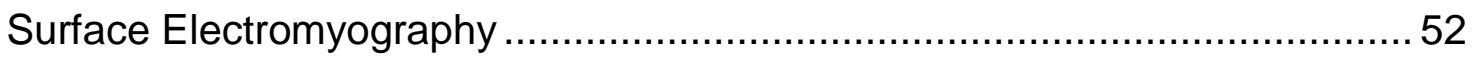




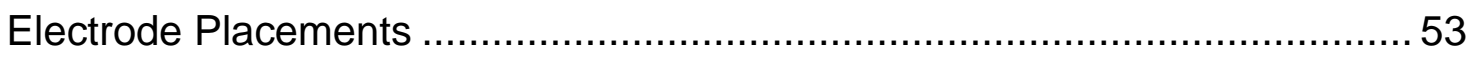

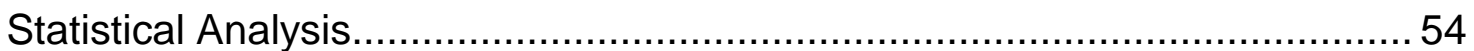

CHAPTER IV: TRUNK MOTOR CONTROL IN TYPICALLY DEVELOPING CHILDREN 55

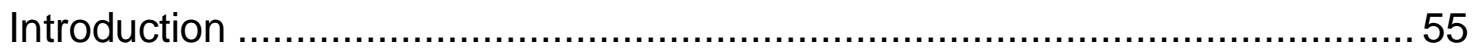

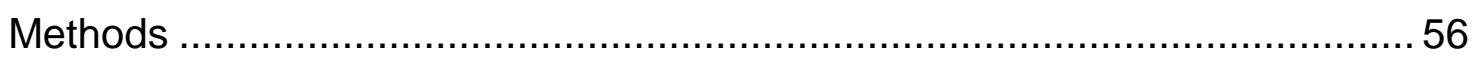

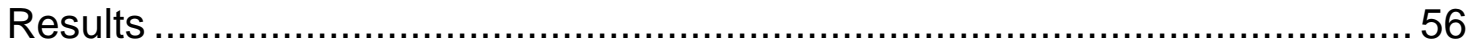

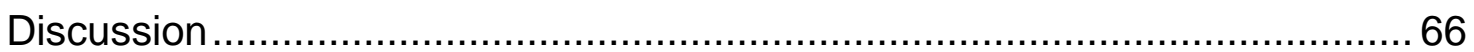

CHAPTER V: RESPIRATORY MOTOR CONTROL IN TYPICALLY

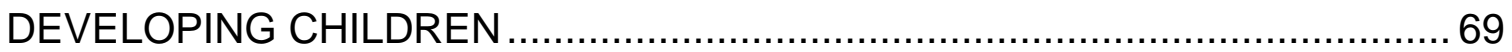

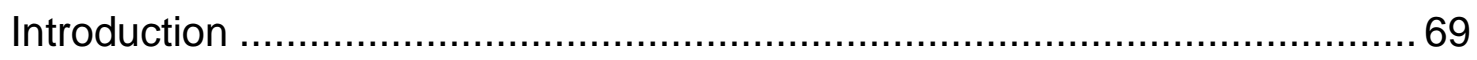

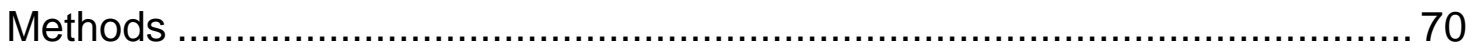

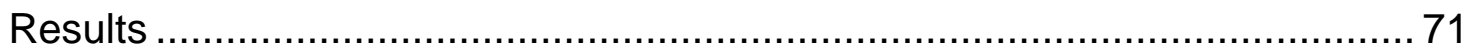

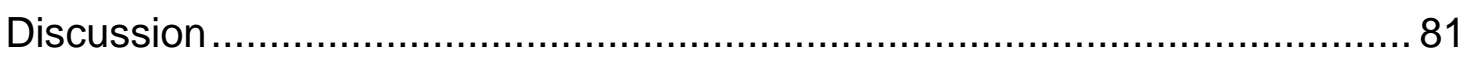

CHAPTER VI: TRUNK MOTOR CONTROL IN CHILDREN WITH SPINAL CORD INJURY 84

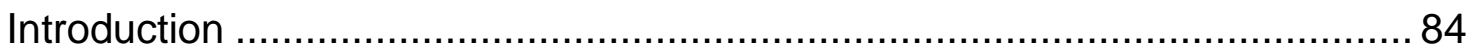

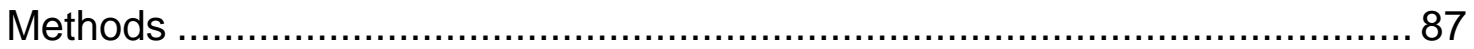

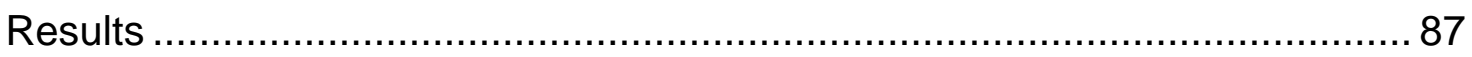

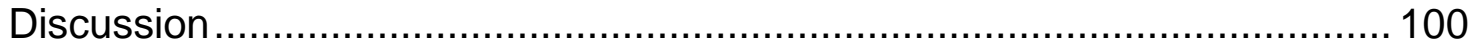

CHAPTER VII: RESPIRATORY MOTOR CONTROL IN CHILDREN WITH

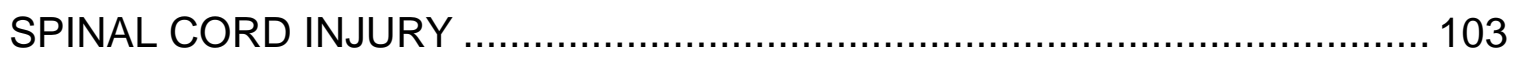

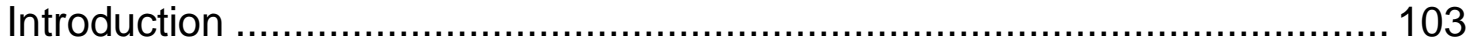

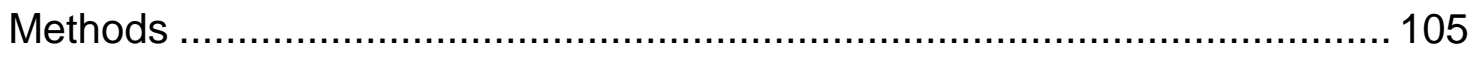

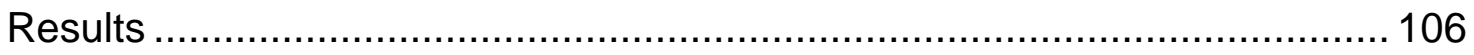

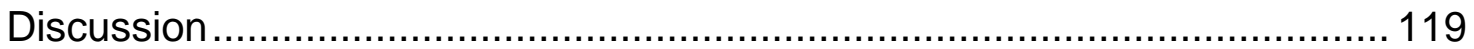

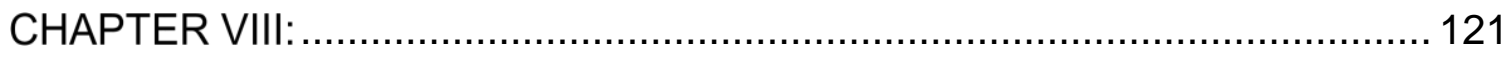

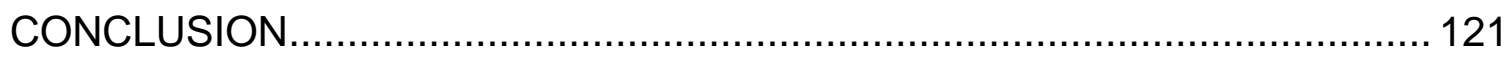

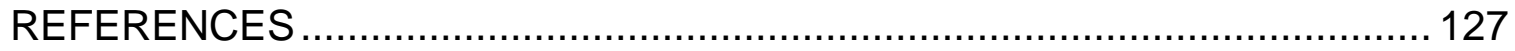

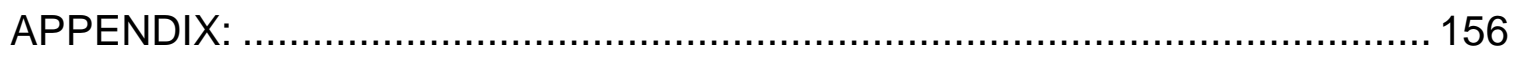

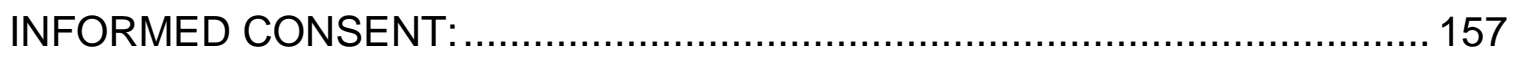

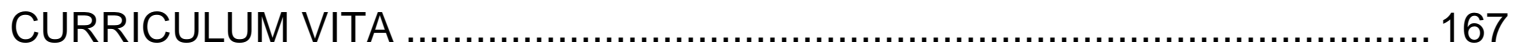




\section{LIST OF TABLES}

Table III-I: Demographics of Typically Developing Children ............................. 37

Table III-II: Demographics of Children with SCI........................................... 38

Table VI-I: Preschool-Typically developing children for respiratory motor control

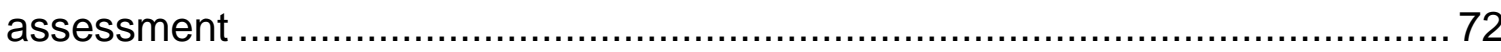

Table VI-II: School-age- typically developing children for respiratory motor control

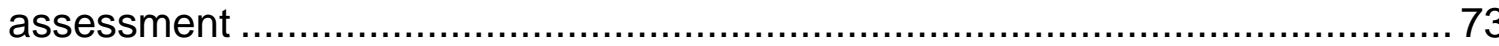

Table VII-I: Preschool-age- Children with spinal cord injury for respiratory motor

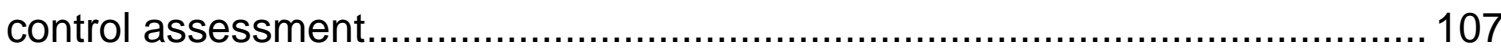

Table VII-II: School-age- Children with spinal cord injury for respiratory motor

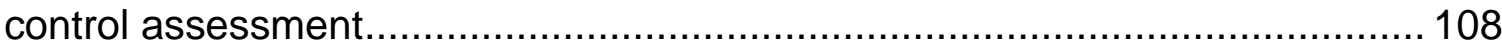




\section{LIST OF FIGURES}

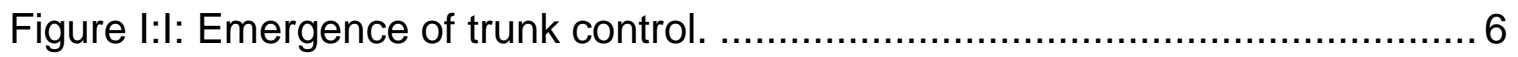

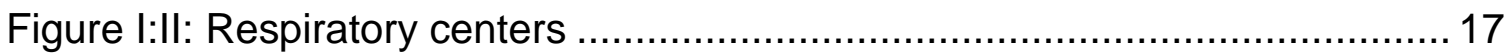

Figure I:III: Respiratory muscles and the spinal levels that innervate them. ....... 22

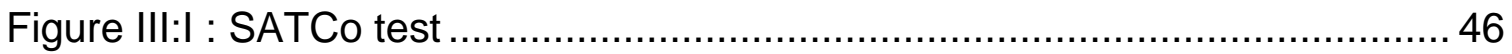

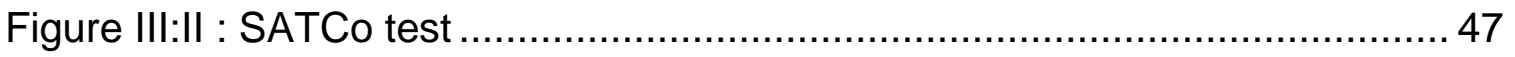

Figure IV:I: Trunk muscle activation during SSC in TD children .......................59

Figure IV:II: Trunk muscle activation during ASC in TD children ......................60

Figure IV:III: Trunk muscle activation during ISSC in TD children .....................61

Figure IV:IV: Trunk muscle activation during OLRSC in TD children .................. 62

Figure IV:V: Trunk muscle activation during BRSC in TD children ................... 63

Figure IV:VI: Trunk muscle activation during PSC in TD children ..................... 64

Figure IV:VII: Trunk muscle activation during NSSC in TD children ................... 65

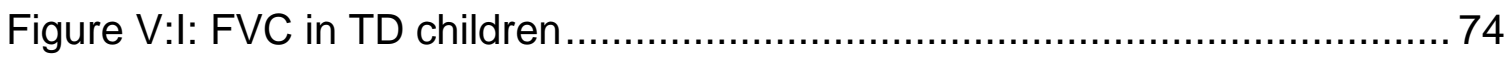

Figure V:II: FVC in preschool and school-age children.................................. 75

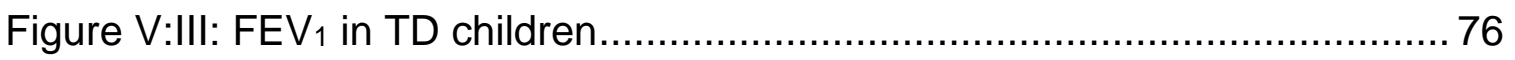

Figure V:IV: $F V_{1}$ in preschool and school-age children ................................ 77

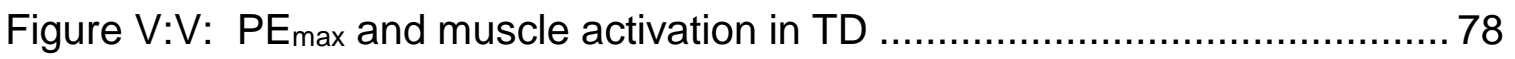

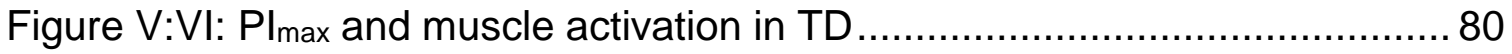


Figure VI:I: Trunk muscle activation during SATCo test in TD children with SCI 89 Figure VI:II: Trunk muscle activation during SSC in TD children with $\mathrm{SCl}$......... 91

Figure VI:III: Trunk muscle activation during ASC in TD children with $\mathrm{SCl}$......... 92

Figure VI:IV: Trunk muscle activation during ISSC in TD children with $\mathrm{SCl}$.......93

Figure VI:V: Trunk muscle activation during PSC in TD children with $\mathrm{SCl}$........ 95

Figure VI:VI: Trunk muscle activation during SATCo test in children with $\mathrm{SCl}$...97

Figure VI:VII: Trunk muscle activation during SATCo in children with $\mathrm{SCl}$......... 98

Figure VI:VIII: Trunk muscle activation during SATCo in children with $\mathrm{SCl}$........99

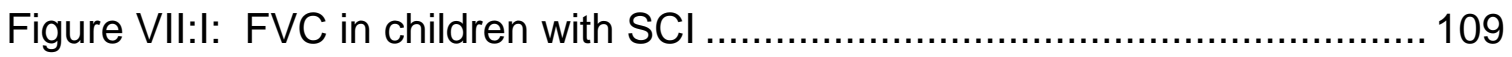

Figure VII:II: FVC in TD and children with SCI........................................ 110

Figure VII:III: FVC in TD and in children with SCI................................... 111

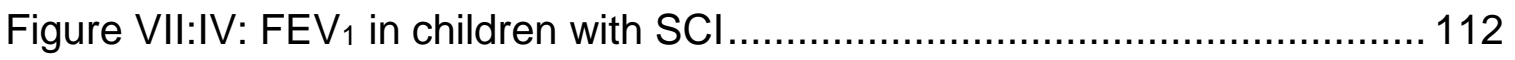

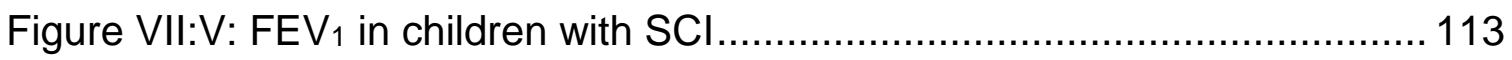

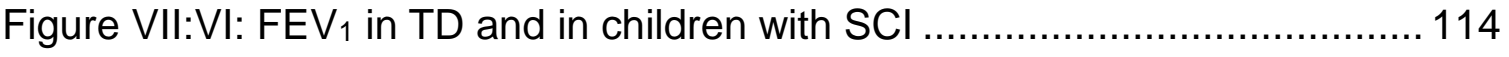

Figure VII:VII: A. PEmax and muscle activation in TD and in children with SCI 115

Figure VII:VIII: A. PImax and muscle activation in TD and children with SCI ... 117 


\section{$\&+\$ 37(5, \quad$ INTRODUCTION}

Pediatric spinal cord injuries pose a unique challenge compared to $\mathrm{SCl}$ in adults because of the continuous physical and cognitive development (Powell \& Davidson, 2015). Motor development is a complex process due to constant interaction between the nervous system and environment (Schmidt, 2011). Any interruption in the normal processes would further complicate it, affecting the typical development of the child. SCI results in loss or impairment of functions, which further leads to reduced mobility and sensation. Independent sitting is a major development milestone and prerequisite for optimal performance of ADLs. Compare to standing and walking, sitting posture takes the relatively larger base of support, but it still requires adequate posture control of the trunk and head. During typical development, posture control of trunk is different for different segments of trunk (Curtis et al., 2015; Rachwani et al., 2013; Sandra L. Saavedra, 2012).

$\mathrm{SCl}$ at the cervical or thoracic level causes impaired trunk control due to paresis or paralysis or spasticity of major trunk muscles, hence, inability to sit

without support (Bjerkefors, Carpenter, Cresswell, \& Thorstensson, 2009; Bolin, Bodin, \& Kreuter, 2000; Potten, Seelen, Drukker, Reulen, \& Drost, 1999) 
Muscles of the trunk have the dual function of maintaining trunk posture and respiration (Paul W. Hodges \& Gandevia, 2000; P. W. Hodges, Gurfinkel, Brumagne, Smith, \& Cordo, 2002). Therefore, motor dysfunction of trunk muscles after $\mathrm{SCl}$ also will affect respiratory functions, with over activation of available accessory muscles of breathing to compensate for paralysis of primary respiratory muscles (A. Ovechkin, Vitaz, de Paleville, Aslan, \& McKay, 2010; Terson de Paleville \& Lorenz, 2015). The higher cervical injury leads to paralysis of most of the intercostal, rectus abdominals, and external oblique muscles. These muscles play an active role during coughing and forced expiratory maneuvers to clear the airways. Respiratory complications like pneumonia and, atelectasis are the leading causes of death among adults and children with $\mathrm{SCl}$ due to inability to cough out secretions and clear airways (Shavelle, DeVivo, Paculdo, Vogel, \& Strauss, 2007; van den Berg, Castellote, de Pedro-Cuesta, \& Mahillo-Fernandez, 2010; van Silfhout et al., 2016).

Neuromuscular scoliosis is prevalent among children with $\mathrm{SCl}$ due to weak or paralyzed trunk muscles and is strongly correlated with age at the time of injury, younger children at a higher risk of developing scoliosis than older children (Mulcahey et al., 2013; S. Parent, J. M. Mac-Thiong, M. Roy-Beaudry, J. F. Sosa, \& H. Labelle, 2011). Nearly all children who sustain SCI prior to skeletal maturity develop neuromuscular scoliosis, which decreases mechanical efficiency of the chest wall, further encumbering lung function (Lancourt, Dickson, \& Carter, 1981; Mayfield, Erkkila, \& Winter, 1981; Mulcahey et al., 2013; Parent, Dimar, Dekutoski, \& Roy-Beaudry, 2010). 
The International Standards for Neurological Classification of Spinal Cord Injury (ISNCSCI) scale is used in clinics to measure the effect and severity of SCI. However, trunk muscles are not included in the assessment (Allen et al., 2009; Chafetz, Gaughan, Vogel, Betz, \& Mulcahey, 2009; S. Parent, J.-M. MacThiong, M. Roy-Beaudry, J. F. Sosa, \& H. Labelle, 2011). Various tests like the Trunk Impairment Scale (TIS), Gross Motor Function Classification System (GMFCS) have been used to measure trunk stability in children and adults. However, independent sitting and standing by participants is a prerequisite for these tests, because testing parameters require that participants be able to sit or stand independently during data collection. Therefore, testing of trunk control in children with low functional level i.e. those who have not achieved independent sitting is limited (Saavedra \& Woollacott, 2015).

Lack of tools to evaluate trunk motor control following $\mathrm{SCl}$ restricts the ability to understand its development and therefore it's challenging to design treatment and strategies to slow down or prevent the progress of long-term effects of SCl. Knowledge about typical development is a prerequisite for the understanding of deviant development; therefore, one of the objectives of this study is to evaluate trunk and respiratory motor control in TD children and compare it to their age-matched children with $\mathrm{SCl}$.

A new tool, the Segmental Assessment of Trunk Control (SATCo) is used in clinics to assess trunk control on a segmental basis and used in TD children who have not developed independent sitting and in children with neuromotor disability (P. B. Butler, Saavedra, Sofranac, Jarvis, \& Woollacott, 2010; Curtis et 
al., 2015). Knowledge about typical development of trunk and respiratory motor control would be of value and a prerequisite for the understanding of deviant trunk development in children with SCI. Therefore, the objective of this study is to evaluate trunk and respiratory motor control in TD children compare their data to age-matched children with SCl.

\section{Background}

\section{Motor Control}

Motor control is defined as a motor task performed by the body using specific mechanisms that regulate movement (Ting \& McKay, 2007). Theories on motor control have tried to explain these complex interactions of various muscles in the body to produce goal-directed movements. Reflex theory of motor control proposed by Sherrington in the late 1900 s explained the complexity of motor control by means of reflexes (Sherrington, 1947). According to reflex theory, complex motor behaviors of the human body are the results of a combination of multiple reflexes. However, this theory failed to explain motor behavior that occurs without the sensory stimulus and motor activities that are occurring too rapidly to allow sensory feedback to influence the outcomes.

The hierarchical theory of motor control suggested a rigid top-down process in which the CNS acts as a command center for all motor tasks. Within the CNS, higher centers such as the motor cortex control or inhibit lower centers, including the midbrain and the spinal cord, and thus dominate movements. In other terms, higher centers are regulating lower centers. However, like some of 
the previous theories, the hierarchical theory fails to explain the reflexive nature of some of the motor activities in the human body, which present with a bottomup control (Figure I.1) (Anne Shumway-cook, 2012; Kenyon \& Blackinton, 2011). Nashner et al, (1985) proposed a hypothesis on the existence of a group of fixed postural synergies, which provides a specific pattern of muscle contraction in agonist and antagonistic muscles (Nashner LM, 1985). However, later studies suggested that postural adjustments during locomotion could vary depending on biomechanical constraints too. In other terms, postural synergies are not fixed; rather there is a flexible organization within the CNS (Hirschfeld \& Forssberg, 1991, 1992). Higher centers responsible for postural control select the appropriate postural adjustments based on biomechanical constraints and underlying motor activity. This selection of postural adjustments could be explained during locomotion where specific postural adjustment are made during walking while different adjustments are made when standing with upper limbs supported (Hirschfeld \& Forssberg, 1991, 1992). Similarly, postural adjustments would shift from ankle strategy to hip strategy as the length of support surface reduces from under the feet during standing (Horak, Nashner, \& Diener, 1990).

The CNS forms an internal representation of the body by receiving appropriate afferent information. During standing, this afferent information also includes alignment /orientation of the body in the vertical position and maintains the center of gravity within the base of support (J. Massion, 1992; Mittelstaedt, 1964; Mittelstaedt \& Fricke, 1988). However, during locomotion, this internal representation is challenged and updated continuously by a feed forward model 
though interaction with efferent signals associated with locomotion. The intact somatosensory (multimodal sensory inflow) system is a prerequisite for selection of proper postural adjustment and formation of the internal representation of the body (Hirschfield and Forssberg 1991). Loss of somatosensory input from lower limbs prevents subjects from inducing the ankle strategy when perturbed, but they can activate hip strategies to compensate for that loss. Similarly, subjects with vestibular impairment can activate the ankle strategy, but not the hip strategy (H. Forssberg \& Hirschfeld, 1994). Much of the studies on trunk motor control have been performed in the standing position. 


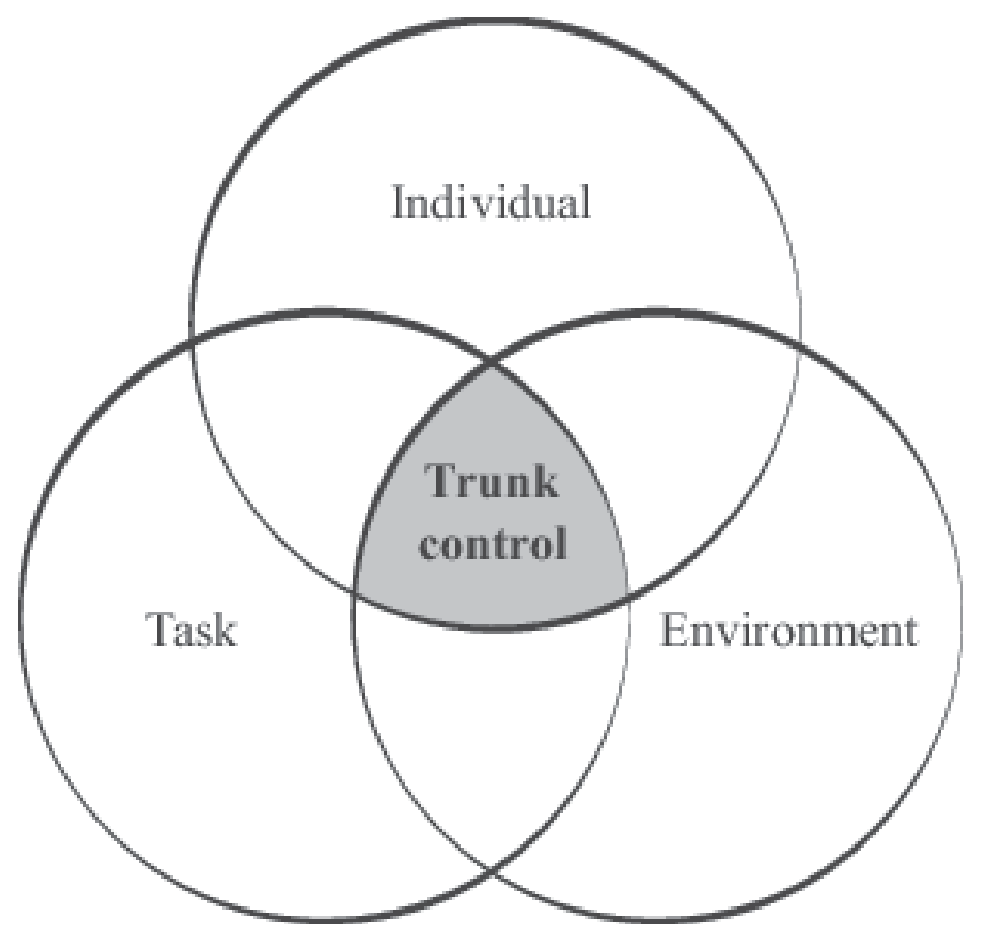

Figure I:I: Emergence of trunk control.

(Adapted from Motor Control (p. 157), by Anne Shumway Cook and Marjorie H. Woollacott, 2017, Philadelphia, PA: 2017 Wolters Kluwer Health. Adapted with permission)

Movement/control emerges through the interaction of individual, the task, and the environment. Development of trunk control requires and varies with the task and environment. According to this system, control of movement not only involves the nervous system, but also the contribution of the musculoskeletal system, as well as the force of gravity (Anne Shumway-cook, 2012). 
Recent work on postural control has suggested two key strategies used by the CNS to produce task-specific movements. A first strategy is a cephaliccaudal approach, involving visual, vestibular and neck muscles to stabilize the head in space and the second strategy, a caudal-cephalic approach, involving touch receptors, pressure receptors and feedback from trunk and extremity muscles. The caudal-cephalic approach is necessary to fix or stabilize a portion of the body that will provide a base of support to allow movement at a different portion of the body (Saavedra \& Woollacott, 2015).

Trunk control is an integral part of the postural control. Trunk control provides stability for both sitting and standing (walking) postural control. It provides a stable base of support for movements of upper and lower extremities. Development of sitting trunk control is a prerequisite for reaching movements. Sitting trunk control is maintained by the continuous complex interaction of trunk muscles. Muscles of the proximal segments provide stability to distal segments to produce a movement like reaching, grasping and lifting (Massion, 1998). However, these studies have not addressed the segmental development (cervical, thoracic and lumbar) of posture within the spinal column, i.e. cervical, thoracic and lumbar (Seevedra, 2010). 


\section{Emergence of Independent Sitting}

As a part of typical development, as infants begin to sit independently, they must learn to balance the background sway of both, head and trunk. This balancing act requires coordination of sensory and motor information from head and trunk segments (Anne Shumway-cook, 2012). Emergence of independent sitting requires continuous coordination of multiple trunk muscles to balance the position of head and trunk during static and reactive states, and maintaining balance during anticipated movements.

The ability to balance head and trunk during steady states occurs at approximately 6 to 8 months of age (Butterworth \& Cicchetti, 1978). Harbourne and Stergius (2003) applied a nonlinear analysis technique to examine posture control during various stages of the development of independent sitting using center of pressure (COP) measurement. Center of pressure is defined as the point where the total sum of a pressure field acts on a body, causing a force to act through that point. They analyzed the COP measurement across three stages of sitting development: Stage 1 included infants with ability hold up head when supported at the trunk (4 to 5.5 months), stage 2 had infants who were able to sit independently for brief periods (10-30s), but not safe to be left in sitting position ( 5 to 6.5 months), and stage 3 , where child sits independently with no risk of fall, but have not started moving in and out of sitting position (6-8 months)

The found that there is a high dimensionality and complexity at stage 1 sitting that decreases as infant progress to stage 2 sitting. This indicates a reduction in the degrees of freedom of the body, as they start to acquire new 
skills. They also reported that dimensionality increases from stage 2 to stage 3 sitting, suggesting an increase in degrees of freedom of head and trunk as infants increase their flexibility in maintaining posture control over the base of support (Anne Shumway-cook, 2012; Harbourne \& Stergiou, 2003). The results from this study indicated that development of sitting postural control in a dynamic process where in infant gradually learns to control the degrees of freedom of head and trunk through three different stages of development of sitting control.

Assessment of sitting trunk control (posture) was of particular interest for this study because of many reasons: the majority of children with $\mathrm{SCl}$ are unable to stand independently, they spend most of their day in this position and developmentally, sitting is attained before standing (MB, 1943). Traditionally, development of posture control in children has been associated with predictable motor behavior, known as "motor milestones." Major milestones include crawling (2 months), sitting (6-7 months), creeping (8-10 months), pull to stand (9-10 months), independent stance (12-13 months), and walking (14-18 months) (Anne Shumway-cook, 2012).

Development is a complex process, involving new skills being learned through continuous interaction with the environment and at the same time dealing with musculoskeletal changes associated with development. Woollacot (1989) described this complex interaction between the neural and the musculoskeletal system as a framework, which includes: 1. Changes in the musculoskeletal system, including the development of muscle strength and changes in relative mass of the different segments; 2 . Development of 
neuromuscular response synergies used in maintaining balance; 3. Development of somatosensory, visual and vestibular systems; 4. Development of sensory strategies to organize these multiple inputs; 5 . Development of internal representations important in the mapping of perception to action; 6 . Development of adaptive and anticipatory mechanisms that allow children to modify the way they sense and move for posture control (Anne Shumway-cook, 2012).

Heinz Prechtl (1986) studied the development of spontaneous head control in neonates by using sEMG signals from neck muscles and simultaneously videotaped their responses. They hypothesized that neonates had poor neck control due to lack of muscle strength to stand against gravity. However, they found no organized patterns of muscle activities and suggested that neonates lack the ability to control their neck not only due to lack of muscle strength but also due to lack of coordinated muscle activities. Other studies have focused on the development of muscle synergies during reactive trunk control (following external perturbations) and muscle synergies during anticipatory balance (reaching movements) (Hedberg, Carlberg, Forssberg, \& Algra, 2005; Hedberg, Forssberg, \& Hadders-Algra, 2004). These studies suggested that infants as young as 1 month could generate direction specific postural adjustment in the neck and the trunk muscles during external perturbation. This direction specific activation of muscles is the first level of control, i.e. the level responsible for generating muscle synergies. However, these muscle synergies or adjustments were uncoordinated (Hedberg et al., 2005; Hedberg et al., 2004; M. Woollacott, Debu, \& Mowatt, 1987). Three months is considered to be the age 
of functional transition, because, at this age, infants start to show spontaneous motor behavior related to postural activities. The presence of the direction specific synergies among neonates suggests that postural adjustment is innate and is present before independent sitting is achieved (Hedberg et al., 2005). Infants between the ages of 5 to 7 months responded with more consistent activation of muscle synergies along with increased tonic activation of agonist and antagonist muscle groups (Hedberg, Schmitz, Forssberg, \& Hadders-Algra, 2007; M. Woollacott et al., 1987). After the age of 8 months, infants start to generate more appropriate adjustment patterns as observed in adults (HaddersAlgra, 2000; M. Woollacott et al., 1987).

The majority of these studies assessed trunk as a single segment, but the trunk is made up of multiple spinal subunits, controlled by different muscles at different levels. In addition, the positions of trunk testing were not standardized in these studies; trunk was allowed to collapse during the test and support was provided to infants who could not maintain sitting position (Bertenthal \& Von Hofsten, 1998; Van der Fits, Otten, Klip, Van Eykern, \& Hadders-Algra, 1999; M. Woollacott et al., 1987). Therefore, the trunk was studied as a whole (single unit) instead of segmental assessments and compensatory movements were acceptable during these tests.

Recent study on the acquisition of trunk control by Saavedra and Woollacott investigated spinal segmental contribution to the development of upright trunk control during typical development of sitting balance (Saavedra \& Woollacott, 2015). They collected longitudinal sEMG and trunk kinematics data 
from a group of 8 TD infants with age range between 3 to 9 months. The segmental contribution of trunk control was assessed at 4 (axilla, midribs, waist, and hips) different levels with pelvic straps used to maintain vertical alignment. An external device was used to support and block any movement at and below the level of support, but allowing full range of motion to the segment above it. They found that trunk control (sitting) develops in a top-down manner, i.e. the cervical control develops first followed by thoracic and lumbar segments. They suggested that trunk control (sitting) in infants develops in four different stages. The first stage is when infants show no control at all, followed by an attempt to initiate the movement (sitting upright), partial control with large sway (wobble), and functional control with minimum sway (adult-like pattern) (Anne Shumwaycook, 2012; Saavedra \& Woollacott, 2015). However, the mechanisms of development of posture control remain an unanswered question.

All the studies mentioned above have examined the sitting posture control in children till they attain independent sitting, standing or walking, but there is a lack of information about how posture control develops further with development after achieving these major milestones. This missing piece is crucial in understanding and comparing the atypical sitting trunk control in children with neuromuscular diseases. According to Woollacott et al, (Anne Shumway-cook, 2012) changes in the musculoskeletal system, which includes the development of muscle strength and changes in relative mass of the different body segments, influence the development of posture control due to continuous interaction with environment and development of new skills. 
Poor trunk control is a characteristic feature among children, adolescents, and adults with cerebral palsy, which results in difficulty in performing ADLs, such as sitting and walking (Bigongiari et al., 2011; De GraafPeters V.B. \& J.; M. H. Woollacott \& Burtner, 1996). However, children with neuromuscular diseases or $\mathrm{SCl}$ also exhibit similar impairment in trunk control and are non-ambulatory. This impairment in the trunk may cause compensatory movements in other segments of the body, which further cause deviation from the typical pattern. Therefore, assessment of trunk control in TD children during sitting could provide valuable information, which may help to understand the impairment of trunk control in children with neuromuscular diseases. 
Respiratory Motor Control in Typically Developing Children

Breathing is a critical behavior that regulates gas exchange to provide support for metabolic demands, maintaining $\mathrm{pH}$ and regulation of body temperature. At rest, breathing remains relatively unchanged, but sleep, exercise, and posture can influence breathing significantly (Feldman \& Del Negro, 2006). Slow changes in breathing are associated with development, diseases, pregnancy, and aging. Breathing is a primal homeostatic neural process, responsible for maintaining normal levels of oxygen and carbon dioxide in blood and tissues.

Respiratory movements (cycle of inspiration and expiration) are continuous rhythmic movements, generated by neural structures located in the brainstem (Levizky, 1995). The spontaneous cycles of inspiration and expiration can be modified, influenced or altered by a number of mechanisms. These mechanisms include reflexes in lungs, airway, and cardiovascular system; receptors in cerebrospinal fluid, command from the hypothalamus, speech center and other areas of the cortex. These mechanisms could, therefore, alter i.e.; increase or decrease the activity of respiratory center to meet the increased metabolic demands of the respiratory system (Levizky, 1995). The neural structure responsible for cyclic respiratory movements is located in the brainstem. This neural circuitry extends from the pons to lower medulla oblongata.

The neural circuits/center that initiate breathing are located in the reticular formation of the medulla (Smith, Abdala, Borgmann, Rybak, \& Paton, 
2013; Smith, Abdala, Rybak, \& Paton, 2009). Neural structures in the pons consist of two centers; pneumotaxic and apneustic centers and neurons within the medulla oblongata are divided into two groups, ventral respiratory group (VRG) and dorsal respiratory group (DRG) (J. E. Butler, 2007; Levizky, 1995). The apneustic center in the pons receives afferent information from the vagus nerve to prevent apneusis, i.e. prolonged inspiratory efforts interrupted by occasional expirations. The pneumotaxic center functions to modulate the activity of apneustic center in the pons. It plays a significant role in fine-tuning the breathing pattern. The VRG on each side of medulla consists of VRG respiratory neurons, which interact with neurons within VRG and pontine nuclei. Inspiratory and expiratory neurons are interlinked within this area of the medulla.

The medullary center does not consist of a discrete "inspiratory or expiratory center". The DRG consist of inspiratory neurons located bilaterally in the nucleus of the tractus solitarius (NTS). These neurons project to the contralateral spinal cord. They serve as the prime initiators of the activity of phrenic nerves. The phrenic nerve in turn supplies to the primary muscle of inspiration- the diaphragm. The ninth (glossopharyngeal) and tenth (vagus) cranial nerves send their afferent projection to the nucleus of the tractus solitarius. These nerves carry information about arterial $\mathrm{PO}_{2}, \mathrm{PCO}_{2}$, and $\mathrm{pH}$ from carotid and aortic chemoreceptors and systemic arterial blood pressure from carotid and aortic baroreceptors (Levizky, 1995). The vagus nerve also carries information from stretch receptors in lungs, which also influences control of breathing. At the spinal respiratory motor neuron level, there is the integration of 
descending signals (influences) and local spinal reflexes that influence these motor neurons. Descending inspiratory neurons excite external intercostal motor neurons and simultaneously inhibit internal intercostal motor neurons by exciting spinal inhibitory interneurons (Levizky, 1995). 


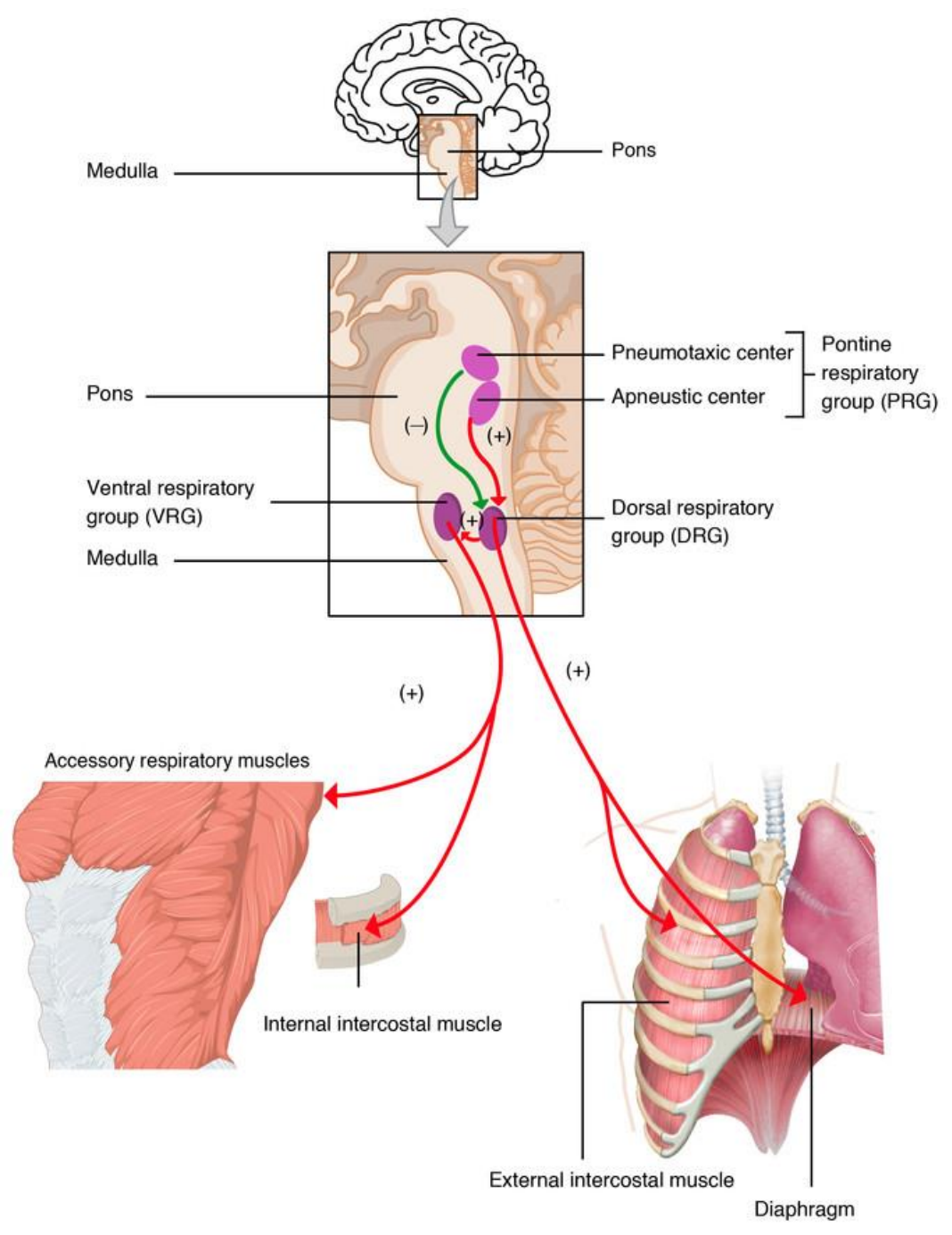

Figure I:II: Respiratory centers

Dorsal respiratory group (DRG) and ventral respiratory group (VRG) of neurons are located in the medulla. Pontine respiratory group (PRG) contains two centers, Pneumotaxic and Apneustic center. Inspiratory neurons from DRG project primarily to the contralateral spinal cord and continue as phrenic nerves and supplied diaphragm (College, 2013). 
VRG contains excitatory and inhibitory interneurons, which receives afferents from nuclei of the solitary tract (NTS), pontine circuits, basal ganglion, cerebellum, hypothalamus, and motor and sensory cortices (Pattinson, Governo, et al., 2009; Pattinson, Mitsis, et al., 2009). VRG of neurons drives both, cranial and spinal motor neurons. Cranial neurons innervate and control the muscles of upper airways, whereas the spinal motor neurons transmit their output to phrenic, intercostal and lumbar motor neurons, innervating diaphragm, thoracic and abdominal muscles respectively (Levizky, 1995; Smith et al., 2013).

\section{Respiratory Motor System}

Respiratory muscles are primarily divided into two main groups, primary muscle (Diaphragm/inspiratory), and accessory muscles of breathing (inspiratory and expiratory). During eupneic breathing, only primary muscles are active. Accessory muscles are recruited during increased respiratory drive, like exercise and respiratory related diseases (Legrand, Schneider, Gevenois, \& De Troyer, 2003; Ratnovsky, Elad, \& Halpern, 2008).

The diaphragm is the primary muscle of inspiration and is innervated by single phrenic nerve on each side of the spinal cord. The diaphragm is a domeshaped muscle with the centrally located tendon. The muscle fibers of diaphragm span from the central tendon to either three lumbar vertebral bodies (crural diaphragm) to the inner surface of lower six ribs (costal diaphragm) (Legrand et al., 2003; Ratnovsky et al., 2008). Contraction of diaphragmatic fibers increases the thoracic volume thereby allowing space for lungs to inflate. At the same time, 
it also increases the intra-abdominal pressure by displacing the abdominal contents caudally.

Intercostal muscles are arranged in two thin layers over intercostal space. Internal and external intercostal muscles are primary muscles of inspiration and expiration, respectively. External intercostals are the outer and thicker layer of intercostal muscles with its fibers oriented obliquely in the caudal ventral direction from rib above to rib below. Contractions of the external intercostal muscles raise and enlarge the rib cage. This action increases the anteroposterior diameter of the chest wall. Internal intercostals form the inner layer of the intercostal muscles with its fibers running in a caudal-dorsal direction from the rib above to rib below. Contraction of internal intercostals causes depression of the rib cage in a manner opposite to external intercostal (A. De Troyer, P. A. Kirkwood, \& T. A. Wilson, 2005). The distribution of motor neurons innervating these muscles is similar. The corresponding intercostal nerve innervates both muscles. The approximate distribution is between T1-T11 (André De Troyer, Peter A. Kirkwood, \& Theodore A. Wilson, 2005; Lane, 2011; Ratnovsky et al., 2008). The abdominal wall is formed by 4 muscles, rectus abdominis, external oblique, internal oblique and transverse abdominis. These 4 muscles are expiratory muscles and are active during active expiration like coughing, sneezing, exercise, speech and singing and in pathological conditions like chronic bronchitis (Ratnovsky et al., 2008).

The rectus abdominis is the most ventral muscle and it runs caudally from posterior aspect of the sternum, $5^{\text {th }}, 6^{\text {th }}$ and $7^{\text {th }}$ costal cartilages to its insertion into the pubic region. The lower thoracic nerves T5-T12 innervate rectus 
abdominis. External oblique is most superficial and originate from $5^{\text {th }}$ to $12^{\text {th }}$ ribs, covers the external intercostal muscles and inserts at iliac crest and linea alba anteriorly. Lower six intercostal nerves innervate the external oblique muscle. Internal oblique muscle runs just beneath external oblique. The muscle fibers originate from inguinal ligament caudally and ascend to insert at linea alba and ribs 10-12. The lower Intercostal, lliohypogastric, and Ilioinguinal nerves supply internal oblique muscle. Transverse abdominis is the innermost muscle of abdominal wall. Fibers of transverse abdominis run circumferentially around the abdominal viscera from the iliac crest, inguinal ligament, thoracolumbar fascia and costal cartilages of $7^{\text {th }}$ to $12^{\text {th }}$ and insert into the xiphoid process, linea alba and pubis. Thoracoabdominal, Subcostal, Iliohypogastric and Ilioinguinal nerves innervate these abdominal (Jacek Cholewicki \& VanVliet Iv, 2002). Contraction of abdominal muscles compresses the abdominal contents against the relaxed diaphragm, forcing it to move cranially into the thoracic cavity. They also help in depressing the lower ribs to deflate the ribcage (Levizky, 1995; Ratnovsky et al., 2008). Accessory muscles of breathing (inspiration) are not involved in eupneic breathing but may be recruited during exercise, inspiratory phase of coughing or sneezing or during pathological states (Mansel \& Norman, 1990).

Sternocleidomastoid, scalene, upper trapezius and pectoralis major are the accessory muscles of breathing. Sternocleidomastoid runs cranially from anterior superior part of manubrium sternum to lateral aspect of the mastoid process. It is innervated by XI cranial nerve (spinal accessory). Scalene muscles are three paired muscles that have their origin from the transverse process of $2^{\text {nd }}$ 
to $7^{\text {th }}$ cervical vertebrae and insert at first two ribs. Contractions of these muscles raise the sternum and first two ribs to increase the volume of the rib cage (André De Troyer et al., 2005; A. De Troyer et al., 2005; Legrand et al., 2003).

Upper trapezius is one of the superficial muscles located at the upper back. It originates from the spinous process of $\mathrm{C}$, external occipital protuberance and nuchal ligament of first cervical vertebra and inserts posterior border of the lateral third of the clavicle. Spinal accessory nerve $(\mathrm{CN}-\mathrm{XI})$ innervates the upper trapezius. Contraction of this muscle elevates both scapulae and is active during forced inspiration tasks (American Thoracic Society/European Respiratory, 2002; Gray, 1918).

Pectoralis major muscle is innervated by cervical $7^{\text {th }}$ and $8^{\text {th }}$ nerve roots and also by $1^{\text {st }}$ thoracic nerve root (Brachial Plexus). It originates from two different ends, clavicular and sternal end. Clavicular end arises from the anterior surface of the sternal half of clavicle and sternal end originates from the anterior surface of sternum and superior 6 costal cartilages. From the site of origin, it runs upward and laterally to insert into the crest if the greater tubercle of the humerus. C5, C6, C7, C8 and T1 nerve roots innervate the muscle. Contraction Pectoralis major can raise $2^{\text {nd }}$ to $6^{\text {th }}$ ribs and it active during forced expiratory maneuvers in people with neuromuscular disorders (De Troyer, Estenne, \& Heilporn, 1986; Gray, 1918). 


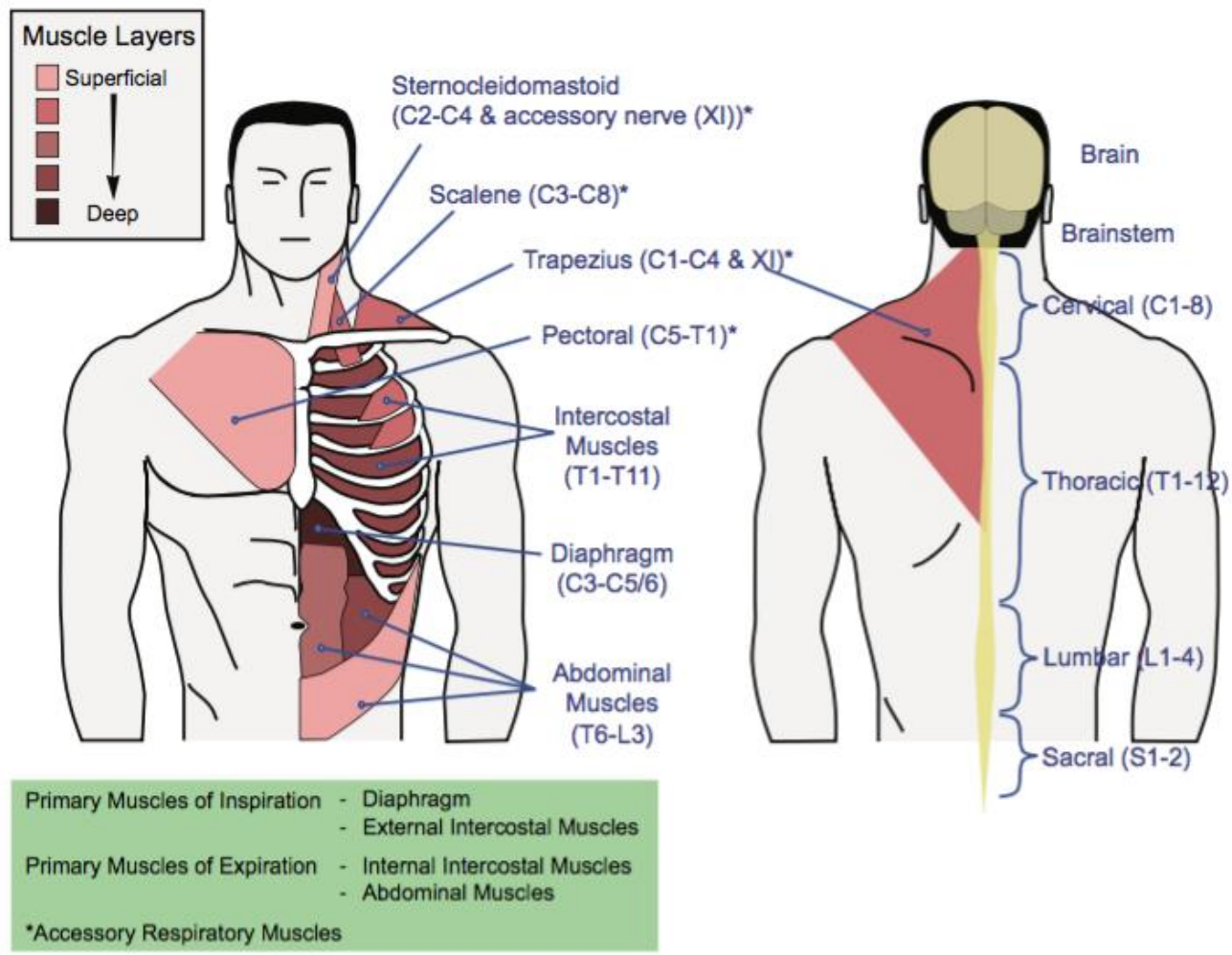

Figure I:III: Respiratory muscles and the spinal levels that innervate them.

Sternocleidomastoid, scalene, trapezius and abdominal muscles are accessory muscles of breathing. The diaphragm is the primary muscle used for inspiration (Elsevier, Michael, et al) 


\section{Spinal Cord Injury}

$\mathrm{SCl}$ is damage to the spinal cord that results in loss or impairment of functions resulting in reduced mobility and sensations. Depending on the severity of the injury, individual will present with both, loss of motor and sensory functions below the level of injury. The higher cervical injury would result in loss of both, upper and lower limb functions including trunk control, resulting in an inability to walk or sit independently. There are about 170,000 new adult traumatic SCI cases in U.S. each year, a cumulative incidence of 54 per million populations (NSCISC, 2016). SCls occurrence is children is uncommon, but it can result in devastating psychological and physiological consequences (S. Parent et al., 2011; Schottler, Vogel, \& Sturm, 2012). The impact of injury at a young age is much greater due to relatively longer life span and interruption of normal development (Schottler et al., 2012).

The etiology of SCl among children is different compared to adults, like lap belt and birth injuries. However, motor vehicle accidents are the leading cause of injury in children. Childhood and adolescent traumatic SCl comprise an estimate of just fewer than $10 \%$ of all new SCl cases, but it poses an enormous cost to the family due to the lifelong need for rehabilitation. Young children who sustain SCI are more likely to have complete injury or paraplegia compared to adults or adolescent who sustain SCI. Children who get injured at an early age are at high risk for secondary complications like, neuromuscular scoliosis and hip dysplasia (Schottler et al., 2012). 
Scoliosis is a three-dimensional deformity of the vertebral column that develops in nearly all children injured prior to skeletal maturity (Lancourt et al., 1981; Mayfield et al., 1981; Parent et al., 2010). The functional impairments after $\mathrm{SCl}$ are variable and depend on the severity of the injury (Powell \& Davidson, 2015; Schottler et al., 2012). People with motor incomplete SCI are more functional than people with motor complete SCI (A. V. Ovechkin, Vitaz, Terson de Paleville, \& McKay, 2013). International Standards examination tests used to classify SCI has two components, i.e. motor and sensory. International Standards for the Neurological Classification of Spinal Cord Injury (ISNCSCI) scale is developed by American Spinal Injury Association (ASIA) is the most widely used scale to determine the severity of the SCI (Kirshblum et al., 2011). ISNSCI scale classifies the injury as cervical (C1-8), thoracic (T1-12), lumbar $(\mathrm{L} 1-5)$ or sacral (S1-5) and myotomes and dermatomes are tested for motor and sensory examination, respectively. ASIA impairment scale is used to grade degrees of impairment. It assigns grades ranging from $A, B, C, D$ and $E$ based on the preservation of sensory and motor function below the level of injury (Kirshblum et al., 2011). However, ISNCSCI scale does not examine the motor function of the entire (muscles). This is a limitation in terms of measuring recovery following $\mathrm{SCl}$. Therefore, an appropriate test should be used to evaluate trunk muscles in people with $\mathrm{SCl}$ as they play an important role in recovery following SCl. 


\section{Development of Trunk Motor Control in Children after SCI}

The primary goal of rehabilitation for people with $\mathrm{SCl}$ is to regain maximum function of upper extremities and to prevent secondary complications. A major proportion of children and adults with SCI perform most of their ADLS (tasks) in seated position. Postural control responsible for independent sitting is a critical part of functional independence in ADLs. However, sitting balance in SCI population is impaired due to the sensorimotor deficit (Potten et al., 1999) and control of trunk muscles become critical because they provide necessary trunk stabilization during sitting. An appropriate postural adjustment is needed to execute a skilled movement and to maintain posture balance during displacement of the body segments. This displacement could be a due force generated internally or exerted by the environment (Potten et al., 1999).

The majority of the research in past have suggested that any voluntary motor activity requires a postural basis, which in turn leads to appropriate postural adjustment during the movement. However, most of these studies examined muscle patterns among individuals who were neurologically intact (Frank \& Earl, 1990; Ghez, Hening, \& Gordon, 1991; Horak et al., 1990; Jean Massion, 1992). Due to paralysis of the majority of trunk muscles, people with $\mathrm{SCl}$ develop new patterns of postural control using the intact sensorimotor system.

Previous studies have indicated that people with higher thoracic SCI compensate for the loss of postural muscle activity by activating non-postural muscles. In patients with high thoracic $\mathrm{SCl}$, latissimus dorsi, pectoralis major and 
trapezius muscle activation during sitting increases in order to compensate for the loss of erector spine (ES) muscle (Potten et al., 1999; Seelen, JanssenPotten, \& Adam, 2001). Although development of alternate postural muscle synergies has been reported in patients with $\mathrm{SCl}$, but these new postural synergies do not fully compensate for the loss of the balance control. Smaller shift in their COP in sitting compared to healthy individuals. In patients with high thoracic $\mathrm{SCl}$, paralysis of trunk muscle is accompanied by impaired somatosensory system, which inevitably leads to impairments in feedback and feedforward control loops necessary for maintain postural control (Jean Massion, 1992; Seelen et al., 2001).

The rehabilitation strategies for patients with $\mathrm{SCl}$ are to regain as much function and control of the upper extremities as possible, to enable them to perform ADLs in sitting position. In this context, muscle of trunk becomes critical, since they provide necessary trunk stabilization (Bjerkefors et al., 2009).Because of the important role of independent sitting in functional independence in ADLs, and its predictive value in the recovery of motor functions following neurological injury, understanding changes in sitting posture control after SCl in developing children is of priority. 


\section{Respiratory Motor Control in Children with SCI}

Pathologies of lung and heart are the primary cause of respiratory disorders (Fishburn, Marino, \& Ditunno, 1990). However, disruption in normal neural regulation of breathing can also have a significant impact on respiratory system (De Vivo, Stuart Krause, \& Lammertse, 1999). Spontaneous ventilation is driven by the respiratory center located in the brain stem and is finely regulated by respiratory muscle performance in response to the respiratory load as described in the section above (Fauroux \& Khirani, 2014).

In non-injured adults, muscles of respiration compensate proportionately to the respiratory load. However, in patients suffering from neuromuscular diseases like $\mathrm{SCI}$, ventilation is compromised, as respiratory muscles are unable to fully overcome the resistance associated with respiration (Brown, DiMarco, Hoit, \& Garshick, 2006). In children, neuromuscular diseases can hamper normal development of the trunk and respiratory muscles and can potentially lead to severe respiratory insufficiency. Understandably, respiratory complications are the leading cause of death among children with SCI (NSCISC, 2016; Parent et al., 2010; Schottler et al., 2012).

Symptoms of respiratory insufficiency are highly correlated with level and severity of spinal lesion. Injury at higher cervical and thoracic cord levels cause paralysis of muscles of respiration (Brown et al., 2006), which directly increase the workload of breathing. The degree of pulmonary dysfunction after $\mathrm{SCl}$ depends on level of injury; with higher cervical injuries causing more damage to respiratory pump than injury at lower thoracic levels (Mansel \& Norman, 1990; 
Roth et al., 1997; Warren, Awad, \& Alilain, 2014). In case of complete high cervical injuries (C1-C3), majority of the muscles involved in breathing are completely paralyzed and patients with such type of injury suffer from acute respiratory failure and eventually become completely dependent of mechanical ventilation (Zimmer, Nantwi, \& Goshgarian, 2008). Injury at mid cervical level (C3-C5) may spare some fibers of diaphragmatic innervation and accessory muscles of inspiration, but patients present with respiratory muscle weakness (inspiratory) and/or fatigue (Lemons \& Wagner, 1994; Mansel \& Norman, 1990; Schmitt, Midha, \& McKenzie, 1991).

Patients with complete C6-C8 injury have intact innervation to diaphragm and accessory muscles of inspiration. Therefore, inspiration is not limited, but they have difficulty in forced expiration maneuvers like coughing and sneezing due to paralysis of intercostal and abdominal muscles. Patients may use pectoralis major as a compensatory medium to facilitate expiration. However, these patients are still at risk of developing respiratory complications due to muscle fatigue at lower resistance to inspiration compared to non-injured subjects (De Troyer, Estenne, \& Heilporn, 1986; Estenne, Knoop, Vanvaerenbergh, Heilporn, \& De Troyer, 1989; Gross, Grassino, Ross, \& Macklem, 1979). Therefore, any respiratory infection could lead to respiratory fatigue and predispose them for respiratory failure. $\mathrm{SCl}$ at thoracic level (T1-T11) will paralyze most of the intercostal and abdominal muscles. This leads to increased abdominal wall compliance, which can compromise the ventilatory capacity and also cause an abnormal rib cage movement associated with 
breathing (Warren et al., 2014). This abnormal movement of rib cage leads to the lower rib cage getting sucked in during inspiration and is called paradoxical breathing (De Troyer, Estenne, \& Vincken, 1986). Paradoxical breathing decreases the tidal volume and causes an increase in residual volume followed by decreased alveolar ventilation (oxygen availability). Therefore, lower lung volumes and decreased availability of oxygen leads to chronic respiratory muscle fatigue because of increased metabolic demand of breathing at rest (De Troyer, Estenne, \& Vincken, 1986; De Troyer \& Heilporn, 1980; Estenne \& De Troyer, 1986). However, injury in children occurring at early ages can result in more severe consequences than similar injuries in adults.

The rib cage in children lacks mechanical efficiency, as it is more circular than elliptical like in adults. This circular shape is due to the attachment of ribs to vertebral column at right angle, which limits the extension/expansion of ribs during inspiration with less tidal volume (Hershenson, Stark, \& Mead, 1989; Openshaw, Edwards, \& Helms, 1984). Higher compliance of the chest wall relative to lung compliance is an inherent characteristic of newborn mammals, which predispose them to have lower functional residual volume. Due to growth and development, there is a progressive increase in the bulk of respiratory muscles, changes in fiber type composition, fiber size and oxidative capacity of diaphragm muscle. In addition, children have less fatigue resistant Type-I fibers, but high proposition of Type-Ilc fatigue susceptible fibers. Therefore, their muscles are prone to earlier fatigue upon movement than adults (American Thoracic Society/European Respiratory, 2002). 
$\mathrm{SCl}$ during this development stage could further limit the normal physiological changes that are needed for optimal respiratory functions. Furthermore, weakness or paralysis of expiratory and abdominal muscles result in retention of mucus due to ineffective or weak coughing (Schilero, Spungen, Bauman, Radulovic, \& Lesser, 2009; Terson de Paleville \& Lorenz, 2015; Vinit \& Kastner, 2009). Mucus retention is the main cause of pulmonary complications like atelectasis and pneumonia in children and adults with $\mathrm{SCl}$ (Claxton, Wong, Chung, \& Fehlings, 1998; Estenne \& Gorini, 1992; Fishburn et al., 1990; Jackson \& Groomes, 1994; Schilero et al., 2009).

SCl-induced immobility and non-weight bearing further worsens their symptoms. Nearly all children who sustain SCI prior to skeletal maturity develop neuromuscular scoliosis, which decreases mechanical efficiency of the chest wall further encumbering lung functions (Mulcahey et al., 2013; Zaba, 2002, 2003a, 2003b). As these children continue to develop and attain maturity, there occur dynamic changes in their musculoskeletal system simultaneously affecting lung volumes and static mouth pressures. In children, strength of respiratory muscles is a function of their age (S. H. Wilson, Cooke, Edwards, \& Spiro, 1984). Considering these factors, children with $\mathrm{SCl}$ are particularly at high risk for developing respiratory complications and it is crucial that these patients are evaluated for respiratory function as early and as frequently as possible.

Currently, pulmonary function testing (spirometry), maximum expiratory pressure $\left(P E_{\max }\right)$ and maximum inspiratory pressure $(\mathrm{PI} \max )$ are used as important tools to diagnose, assess and manage respiratory diseases, both in 
adults and children. However, assessments using these tools fail to provide information about underlying neural drive to the respiratory motor system (A. Ovechkin et al., 2010; Terson de Paleville \& Lorenz, 2015). Unfortunately, there is profound lack of knowledge about development of respiratory motor control in neurologically intact children. Correlation of respiratory motor control with the development of the CNS is also largely unidentified. These gaps in our knowledge about development of neural control act as a barrier to treat $\mathrm{SCl}$ induced respiratory insufficiency in developing children.

In adults, research has been conducted to evaluate respiratory muscle activation by using respiratory motor control assessment protocol (S. C. Aslan, M. K. Chopra, W. B. McKay, R. J. Folz, \& A. V. Ovechkin, 2013; Leung et al., 2012; A. Ovechkin et al., 2010; Terson de Paleville \& Lorenz, 2015). In the absence of similar research conducted in children, neurologically intact or not and acknowledging the significance of neural control in development of respiratory motor system, first aim of this study is to evaluate respiratory neural drive in neurologically intact, TD children by using standard pulmonary function testing in a multi-muscle surface electromyography model and second aim is to evaluate these functions in children with $\mathrm{SCl}$ and compare their results to TD children. 


\section{$\&+\$ 37(5,, \quad$ HYPOTHESIS AND SPECI) ,\& \$,0 6}

To understand the mechanisms behind atypical/abnormal trunk motor control in children with $\mathrm{SCl}$ we needed a trunk control model similar to that used in TD children. Due to significant musculoskeletal changes (height, weight, muscle girth) associated with age, we decided to form two experimental groups of TD children: i.e. preschool (3-5 years) and school-age (6-13 years). The overall goal of this dissertation is to investigate the impact of age on trunk and respiratory motor control outcomes and to understand the underlying mechanisms of abnormal trunk and respiratory motor control in children with SCI.

Our first Specific Aim was to establish normative, age-dependent (3-13 years) neurophysiological trunk motor control outcome measures in neurologically intact, healthy children. We hypothesized that the neurophysiological characteristics of trunk motor control depend upon age: - i.e. older children (6-13 years) would demonstrate higher sEMG amplitude than children in younger age group (3-5 years).

Our second Specific Aim was to establish normative, age-dependent (313 years) neurophysiological respiratory motor control outcome measure in TD children. We hypothesized that children in older age group produce higher lung 
volumes and airway pressures associated with higher sEMG amplitude than children in the younger group.

By understanding about the development of trunk and respiratory motor control in TD children, our final Specific Aim was to compare this trunk and respiratory motor control outcomes in TD children to age-matched, children with $\mathrm{SCl}$. We hypothesized that neurophysiological characteristics of trunk and respiratory motor control outcomes in children with $\mathrm{SCI}$ depend on the current age and neurological level and severity of $\mathrm{SCl}$ i.e. children with $\mathrm{SCI}$ would demonstrate lower sEMG amplitude when compared to age-matched TD children. Also, children with higher levels of SCI would produce lower SEMG amplitude (trunk and respiratory motor outcomes measures) when compared to children with lower levels of SCl. 


\section{$\&+\$ 37(5,,, \quad$ METHODS AND MATERIAL \\ Inclusion Criteria}

Inclusion criteria for TD group included as follows: age between 3 to 13 years; stable medical condition; no known neurological or musculoskeletal disease/abnormalities, able to follow age-appropriate instructions. Inclusion criteria for children with $\mathrm{SCI}$ were as follow: $\mathrm{SCI}$ participants were between 3 to 13 years of age; stable medical condition; no painful musculoskeletal dysfunction, no;-unhealed fractures; no pressure sores or urinary tract infections that might interfere with testing; non-progressive SCI classified by American Spinal Injury Association Impairment Scale (AIS) or other non-progressive neurological conditions as outlined above; not ventilator dependent and absence of cardiovascular or pulmonary diseases, endocrine disorders, or other major medical illness contraindicated for respiratory testing.

Exclusion criteria for TD children and children in SCI group were as follow: unstable medical condition; upper respiratory tract infection in past two weeks; acute or chronic respiratory diseases; any major systemic diseases like cardiac or renal problems, ventilator dependent; and other medical illness contraindicated for respiratory tests. 


\section{Recruitment}

Children with SCI were recruited from Frazier Rehabilitation Institute, Louisville, Kentucky. Once a parent/legal guardian expressed interest in having their child participate in this study, their information was added to the secure database. The research team utilizes the Human Locomotion Research Center's potential volunteer database (UofL Study\# 06.0647). Volunteers were invited to the Frazier Rehab Institute to meet Dr. Andrea Behrman and her research team. The study was approved by the IRB at the University of Louisville (IRB\#15.0585). The experimental assessment procedures were explained to volunteers and the consent was obtained. All participants were encouraged to read the informed consent guidelines given by Dr. Behrman/research staff and to discuss it with their physician, family, and friends, before agreeing to enter the study.

Flyers describing the study were also provided to families upon entry into routine care and contact information of study personnel provided for parents to call or contact if they were interested in learning more about the study. Additionally, flyers for non-injured healthy children were posted in different buildings of University of Louisville campuses at Belknap and Health Science centers to search for potential research volunteers based on eligibility criteria. 


\section{Participants}

A total of 16 TD children ( $9 \mathrm{~F} \& 7 \mathrm{M}$ ) participated in the study with 5 children in the preschool group and 11 children in school age group. There was a total of 14 children with SCI who participated in this study, with 8 in the preschool group and 6 in school age group. The mean age of children in TD and SCI group were $7(7 \pm 2$, Mean $\pm S D)$ and $5(5 \pm 2)$ years, respectively. Demographics of TD and $\mathrm{SCl}$ participants are shown in Table I.I and Table I.II respectively. 
Table I-I: Demographics of Typically Developing Children

\begin{tabular}{|c|c|c|c|c|}
\hline Subject ID & Age (years) & Gender & Height $(\mathrm{cm})$ & Weight (kg) \\
\hline N149 & 3 & $\mathrm{~F}$ & 89 & 17 \\
\hline N133 & 4 & $M$ & 98 & 17 \\
\hline N150 & 4 & M & 114 & 20 \\
\hline N130 & 5 & $\mathrm{~F}$ & 114 & 27 \\
\hline N134 & 5 & $M$ & 106 & 17 \\
\hline N126 & 6 & M & 101 & 17 \\
\hline N145 & 6 & $\mathrm{~F}$ & 114 & 27 \\
\hline N110 & 7 & $\mathrm{~F}$ & 122 & 27 \\
\hline N127 & 8 & $\mathrm{~F}$ & 124 & 23 \\
\hline N148 & 8 & $\mathrm{~F}$ & 157 & 75 \\
\hline N147 & 9 & $\mathrm{~F}$ & 144 & 37 \\
\hline N146 & 10 & $\mathrm{~F}$ & 137 & 51 \\
\hline N144 & 10 & $\mathrm{~F}$ & 129 & 33 \\
\hline N108 & 11 & M & 145 & 27 \\
\hline N128 & 11 & M & 147 & 36 \\
\hline N109 & 12 & M & 160 & 44 \\
\hline Mean \pm SD & $7 \pm 2$ & 9F\&7M & $125 \pm 20$ & $30 \pm 15$ \\
\hline
\end{tabular}


Table I-II: Demographics of Children with SCI

\begin{tabular}{|c|c|c|c|c|c|c|}
\hline Subject ID & $\begin{array}{l}\text { Age } \\
\text { (years) }\end{array}$ & Gender & $\begin{array}{l}\text { Height } \\
(\mathrm{cm})\end{array}$ & $\begin{array}{l}\text { Weight } \\
\text { (kg) }\end{array}$ & $\begin{array}{l}\text { Injury } \\
\text { Level }\end{array}$ & $\begin{array}{l}\text { Time Since Injury } \\
\text { (Months) }\end{array}$ \\
\hline P12 & 3 & $M$ & 91 & 11 & L1-2 & 15 \\
\hline P3 & 4 & $M$ & 97 & 14 & $\mathrm{~T} 2$ & 50 \\
\hline P7 & 4 & $\mathrm{~F}$ & 114 & 20 & $\mathrm{C} 5$ & 30 \\
\hline P8 & 4 & $\mathrm{~F}$ & 112 & 28 & $\mathrm{C} 5$ & 58 \\
\hline P15 & 4 & $\mathrm{~F}$ & 104 & 16 & $\mathrm{~T} 12$ & 12 \\
\hline P9 & 5 & $F$ & 114 & 23 & $\mathrm{~T} 2$ & 11 \\
\hline P14 & 5 & M & 101 & 15 & NC & 28 \\
\hline P16 & 5 & $M$ & 109 & 19 & $\mathrm{~T} 12$ & 17 \\
\hline P13 & 6 & $M$ & 118 & 21 & T3 & 7 \\
\hline P4 & 6 & $\mathrm{~F}$ & 101 & 24 & $\mathrm{C} 8$ & 73 \\
\hline P6 & 7 & $M$ & 124 & 24 & $\mathrm{~T} 8$ & 41 \\
\hline P1 & 9 & $M$ & 148 & 31 & $\mathrm{~T} 1$ & 69 \\
\hline P10 & 9 & $M$ & 141 & 30 & $\mathrm{C} 5$ & 113 \\
\hline P5 & 10 & $\mathrm{~F}$ & 137 & 32 & $\mathrm{C} 5$ & 129 \\
\hline Mean \pm SD & $5 \pm 2$ & $6 F \& 8 M$ & $115 \pm 16$ & $22 \pm 6$ & NA & $46 \pm 36$ \\
\hline
\end{tabular}




\section{Equipment}

Custom written acquisition software (National Instruments, Austin, TX) was used to acquire sEMG signals. We used MA300-XVI 16-channel sEMG system (16 full bandwidths, a ground electrode, Motion Lab Systems, Inc., Baton Rouge, LA) with preamplifier electrodes placed directly onto skin surface located above the muscles belly in the direction of muscle fibers. For the measurement of FVC and FEV 1 , a CPFS/D USB spirometer and Breeze Suite System (MGC Diagnostics, St. Paul, MN) with birthday candles as incentive was used. Spirometer was connected to a mouthpiece. Breeze software calculated the percent-predicted values of FVC and $\mathrm{FEV}{ }_{1}$ for both TD and children with $\mathrm{SCl}$, based on their height, weight, gender, ethnicity, and age (Beydon et al., 2007; Eigen et al., 2001; Miller et al., 2005; A. Ovechkin et al., 2010).

Airway pressures ( $P E_{\max }$ and $\mathrm{PI}_{\max }$ ) were measured by using MP45; lowpressure transducer system (Validyne Engineering, Northridge, CA) which was connected to a T-piece monitoring circuit. T-piece consisted of a mouthpiece, which was used by participants to breathe in and out and a one-way valve hat offered resistance to expiration during PEmax and to inspiration during PImax measurements. There was a small leakage of $1.5 \mathrm{~mm}$ in diameter to prevent activation of buccal muscles and glottis closure during the event (A. Ovechkin et al., 2010).

During postural testing, children sat on a custom-made bench with adjustable height and the back support surface. The backrest was used only during respiratory assessment and was taken off during postural testing. 


\section{Clinical assessment}

Parents or legal guardians of children signed the Informed consent following approval from the institutional review board. Children above the age of seven years signed assent forms. Fourteen children with chronic SCl were recruited from physical therapy unit at Frazier Rehabilitation Center, Louisville. Sixteen TD or neurologically intact children who had no history of any respiratory or cardiovascular dysfunction were assessed for normative age-

matched data. Children with SCI were classified using the American Spinal Injury Association Impairment Scale (AIS) table 1.2. However, some children were too young to have a conclusive AIS score. All SCI children were undergoing locomotor training at Frazier Rehabilitation Center. Participant's demographics are shown in table III.1

\section{Posture Motor Control Assessment}

There are about 19 different tests to measure trunk control, seated posture control and functional abilities for children with motor impairments (Field \& Livingstone, 2013). However, independent sitting or standing by participants is a prerequisite for these tests and require that participants be able to sit or stand during data collection.

For this study, we used Segmental Assessment of Trunk Control test (SATCo). This test is used in clinics to assess sitting trunk control on a segmental basis for TD children, children who have not developed independent sitting and for children with cerebral palsy. SATCo is a reliable and valid measure of trunk control in TD children or children with neuromuscular disabilities (P. B. 
Butler et al., 2010). The test has been used in pediatric clinics to measure precise control of sitting balance at various levels of support (P. B. Butler et al., 2010; MB, 1943; Rachwani et al., 2013; Sandra L. Saavedra, 2012).

For SATCo measurement, trunk control is examined with therapist progressively changes the level of support from top at shoulder girdle and axilla to assess cervical (head) control, inferior scapula (mid-thoracic control), lower ribs (lower thoracic control), below ribs (upper lumbar control), pelvis (lower lumbar control) and no support, to measure full trunk control (Figure 4-I \&II). The test measures three aspects of trunk control, static (stationary) control, active (anticipatory) and reactive (external perturbation) (P. B. Butler et al., 2010). During static control testing, the participant was asked to look straight in front while maintaining an upright posture for at least 5 seconds. Therapist counted the numbers from 1 to 5 to let the participant know when to stop or rest. Static scores are given if the participant can maintain neutral trunk posture above the level of support for at least 5 seconds.

During active control testing, the participant was given two targets, one on each side, left and right. Instructions were given to maintain the upright posture and turn head to the right to look at the target, come back to the middle and turn head to look at the target on left and come back to the middle. Active control is scored if the participant can maintain an upright posture during head movements. During reactive control, the external perturbation is provided in all four directions (front, back, right and left) using two fingers. In addition, the point of perturbation remains horizontal for all the levels of trunk control. Reactive controlled is scored 
if the participant can maintain the trunk position in neutral after external perturbation (P. B. Butler et al., 2010; Saavedra \& Woollacott, 2015).

We used a pressure sensor mounted on two fingers to perturb the participant in all four directions. The sensor was connected to Labview acquisition computer, which helped us to know the exact time of impact on child's body. The SATCo test scores all three controls with remarks as either present, absent or not tested (NT). As the level of support is lowered from full support at the shoulder to no support at all, the stability of trunk is challenged progressively. Therefore, participant's ability to maintain an upright posture and quickly come back to regain that trunk stability is tested progressively for static, active and reactive control.

Accurate scoring of the test depends on various factors: 1 . Adequate alignment and extension of the trunk by supporting therapist. 2. Note any compensatory strategies used by the participant, especially trunk alignment and hand placement. 3. Accurate hand placements by therapist i.e. accurately determine the anatomical landmarks in participant's immature skeletal structure (adipose tissue, ribs not elongated at a young age). One of the advantages of using SATCo is that it could be used to assess trunk control in children who have developed sitting trunk control as well as in children who have not achieved any level of sitting trunk control. 


\section{Surface Electromyography}

sEMG is the study of muscle function through the inquiry of the electrical signal the muscle emanates (Basmajian Jv, 1985). In 1849, Du Bois Reymond was first to record electrical activity produced by muscle during voluntary contraction. Later in 1917, Pratt demonstrated that the amplitude of the energy produced during muscle contraction was due to the recruitment of the individual muscle fibers, and not due to the size of neural impulse (FH, 1917).

The CNS produces motor unit action potentials in the muscles. These action potentials create electrical potential differences in the muscles. These electrical potential differences can be measured using electromyography. Due to continuous improvement in EMG recording instruments, researchers began to use non-invasive surface EMG (sEMG).

The sEMG recording is a safe, non-invasive and easy method to record the electrical activity of the underlying muscle. This electrical activity (potential difference) provides an objective quantification of the muscle strength. The electrical potential differences can be reported as EMG amplitude. EMG amplitude is the sum of the electrical potential differences within a muscle produced by the active motor units in the vicinity of the electrodes on the skin. sEMG amplitude provides a global measure of overall motor activity during the muscle action being performed or tested. The sEMG activity for each muscle is calculated using a root mean square (RMS) algorithm (American Thoracic Society/European Respiratory, 2002; Sherwood, Graves, \& Priebe, 2000) which produce a mean value in microvolts $(\mu \mathrm{V})$ (Gary Kamen, 2010; Meekins, So, \& 
Quan, 2008; Merletti, Rainoldi, \& Farina, 2001). The amplitude is estimated using RMS values, which coincide with a standard deviation of distribution (Merletti et al., 2001).

sEMG is used in both clinical and research settings to study and diagnose various neurological disorders. Data from sEMG could be used to assess the extent and severity of the neurological injury. sEMG studies have shown to be helpful to track recovery, the effectiveness of therapies, and plastic changes after spinal cord injuries (Dietz, Colombo, Jensen, \& Baumgartner, 1995; Gutierrez A, 2006; Harkema et al., 2011).

Researchers have suggested that there is a linear relationship between airway pressure and sEMG output from respiratory muscles associated with that airway pressure (Yokoba, Abe, Katagiri, Tomita, \& Easton, 2003). Also, there is a strong correlation between pulmonary function and neural activation of respiratory muscles (Cerqueira \& Garbellini, 1999; Fujiwara, Hara, \& Chino, 1999; Nobre et al., 2007; Yokoba et al., 2003). However, not all respiratory muscles show this linear relationship. Respiratory motor control assessment (RMCA) protocol, which includes assessment of sEMG from major respiratory muscles, is a valid method to quantitatively evaluate the respiratory motor function in neurologically intact and people with SCI (Cerqueira \& Garbellini, 1999; Fujiwara et al., 1999; Nobre et al., 2007; A. Ovechkin et al., 2010; Terson de Paleville \& Lorenz, 2015; Yokoba et al., 2003). 


\section{Respiratory Motor Control Assessment}

Pulmonary function testing is an important diagnostic tool used in the management of respiratory diseases in adults and children (American Thoracic Society/European Respiratory, 2002; Eigen et al., 2001; Levizky, 1995; Miller et al., 2005; Nicot et al., 2006). Spirometry is frequently used in the pediatric population, especially in children with muscle dystrophies, asthma, and pulmonary fibrosis (Crenesse, Berlioz, Bourrier, \& Albertini, 2001; França et al., 2016; Kanengiser \& Dozor, 1994; M.R. Miller, 2005; Vilozni, Barker, Jellouschek, Heimann, \& Blau, 2001).

During RMC assessment, spirometry and airway pressures were recorded in sitting position with hip and knee flexed to 90 degrees. Participants were asked to sit on a bench with back supported. We recorded sEMG from various respiratory muscles while the participant performed pulmonary function testing. Age appropriate instructions were given to participants during PFT. None of the participants had any previous experiences of performing spirometry. Only one examiner instructed all participants for PFT assessments and since all participants learn differently, procedures were explained accordingly.

Before the event, examiner demonstrated the procedure using his own mouthpiece. Each event was performed three times with one-minute rest period between them. Poor attempts were excluded and we repeated them to get at least three consistent good attempts. The sEMG from various respiratory muscles were recorded during these events. 


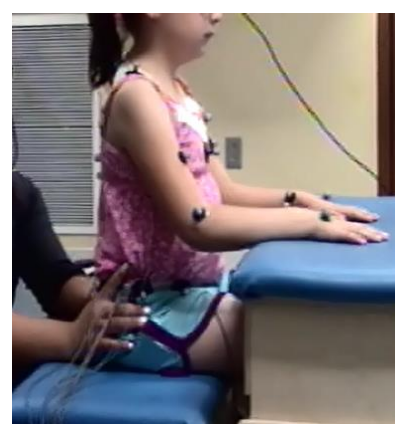

A

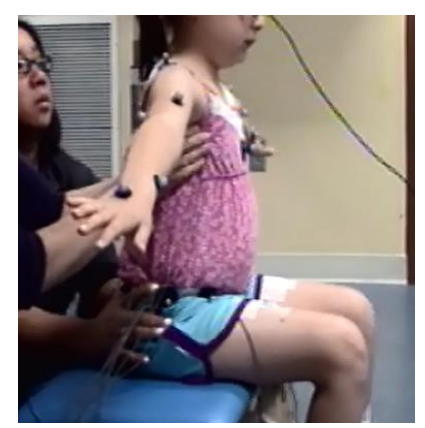

B

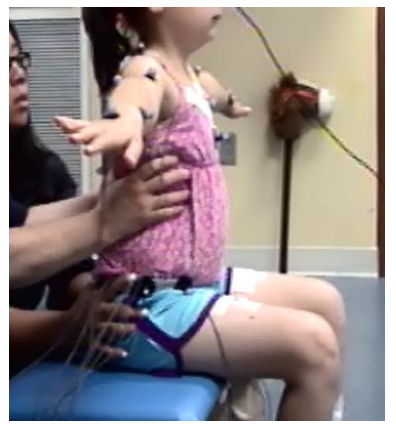

C

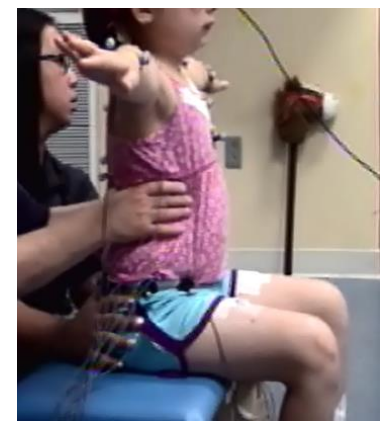

D

Figure III:I : SATCo test

Segmental support provided at four different levels to test for Cervical, thoracic and upper Lumbar spinal segment.

A. Support is provided at shoulder girdle with pelvis maintained in neutral position by another therapist. B. Support at Axilla level to test for cervical segmental control. C. Trunk supported at Inferior scapula level to test for lower cervical control. D. Therapist provided support at over lower ribs level to test for the upper thoracic segment. 


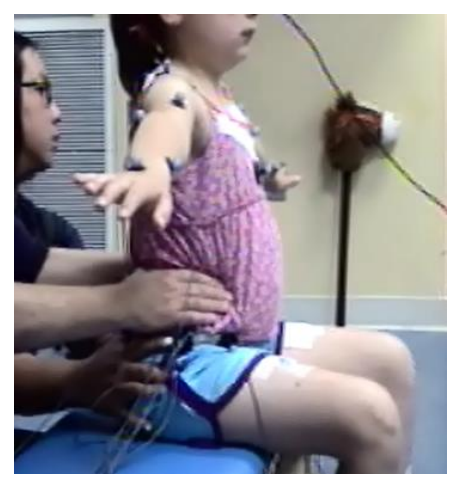

E

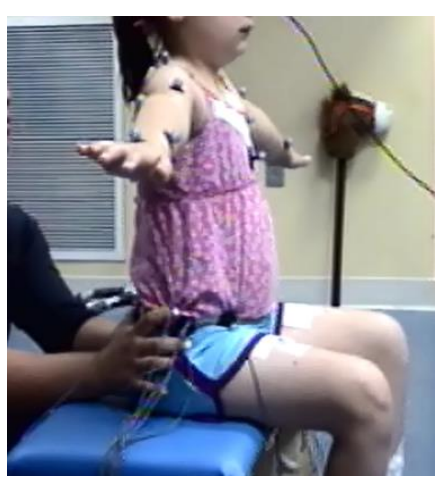

$\mathbf{F}$

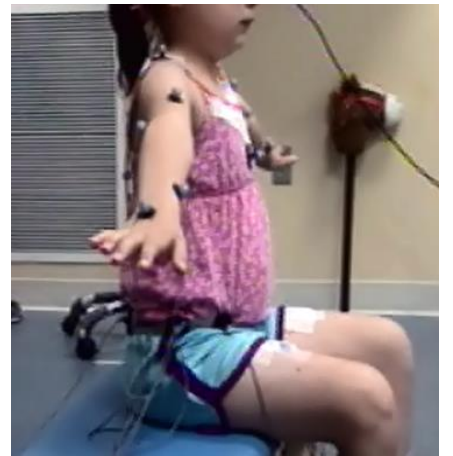

G

Figure III:II : SATCo test

Segmental support provided at three different levels to test for thoracic and upper Lumbar spinal segment. E.

Support is provided at below ribs level with pelvis maintained in neutral position by another therapist. F. Support at

Pelvis only to test for lumbar segmental control. G. Trunk supported by the child to test the stability of entire trunk. 


\section{Spirometry}

Measurement of lung function is important to understand respiratory physiology and for clinical assessment. For the past fifteen years, American Thoracic Society (ATS) and European Respiratory Society (ERS) have been working in the area of pulmonary function testing to prove its usefulness in clinics and as well as in research.

Spirometry is a physiological test that measures lung function by measuring velocity and volume of air a person can breathe in and out of their lungs in one breath. The primary measurement, in this case, is the volume of air. Normally during inhalation, air moves freely through the trachea, bronchi, and bronchioles and finally through smaller sacs called alveoli. Small blood vessels called capillaries surrounds alveoli. Oxygen from the inhaled air diffused through capillaries, while carbon dioxide from body diffuses out of capillaries and into alveoli, which is then exhaled out during expiration. Diseases such as asthma, pulmonary fibrosis and emphysema and neuromuscular diseases could affect the amount of air breathed in or diffused through alveoli, thereby reducing the amount of oxygen in the blood and finally leads to fatigue.

Spirometry is indicated to evaluate symptoms, signs, measure the effect of disease on pulmonary function, pre-operative risk, assess prognosis or to assess health status before beginning strenuous physical activity programs (Leung et al., 2012; Miller et al., 2005).

Prior to testing, all children were measured for their height (inches) and weight (lbs.). American Thoracic Society (ATS) guidelines were followed for 
standard spirometry measurements. Breeze suite system 2007 with incentive computer games was used to measure forced vital capacity (FVC), forced expiratory volume in one second (FEV 1 ), FEV 0.75 , FEV 0.5 AND peak expiratory flow (PEF). All these values were expressed as a percentage of predicted values for each child. Demographic information related to age, gender, ethnicity, height and weight was added in the software to calculate their predicted values. Testing was performed in a child-friendly environment with colorful paintings and toys to play with while they were getting ready for the procedure. Instructions were modified and demonstrated for each child (Eigen et al., 2001) as follows:

1. Participants in this study were ranging from age 3 to 13 years therefore, instructions were kept simple and age appropriate.

2. Adequate time was given to each participant to feel safe and get used to the surroundings. Age appropriate toys, reading the material, movies etc. were available to keep them entertained while sEMG electrodes were placed.

3. First, the mouthpiece and nose clip were introduced to the participant and then the procedure was explained by stating that they need to take a big breath in and blow out as fast as possible and keep blowing as long as they can.

4. The examiner demonstrated the procedure to each participant using a separate mouthpiece and asked the participant to watch and follow. 
5. The participant was then asked to place the mouthpiece their mouth and practice to breathe at tidal volume, and then take a big breath in, and blow out as fast as possible. We used birthday candles as an incentive for every child and they were encouraged to blow all the candles in one single exhale (or to their maximum capacity) (Vilozni et al., 2001). Testing was repeated until three acceptable spirograms were obtained or within the 15 minutes limit (Miller et al., 2005).

6. All participants were tested in sitting position with back supported and hip and knee flexed to 90 degrees. The flow-volume curves were later further analyzed by the examiner and were accepted or rejected based on ATS criteria for pulmonary testing in children (Crenesse et al., 2001). The highest values for FVC and FEV 1 were included for statistical analysis.

$$
\text { Maximum Expiratory and Inspiratory Airway Pressure }
$$

Static mouth pressures, maximum inspiratory pressure $(\mathrm{PI} \max )$ and expiratory pressure $\left(\mathrm{PE}_{\max }\right.$ ) measurements $\left(\right.$ in $\mathrm{cmH}_{2} \mathrm{O}$ ) are used to estimate the strength of respiratory muscles (Leung et al., 2012). We measured these airway pressures while simultaneously recording sEMG signals from various respiratory muscles. PImax and PEmax were recorded using differential pressure transducer (MP45-36-871-350) UPC 2100 PC card from Validyne Engineering (Northridge, CA). Both $\mathrm{PI}_{\max }$ and $\mathrm{PE} \mathrm{max}_{\max }$ were performed in sitting upright position with hip and knee joint flexed to 90 degrees.

$\mathrm{Pl}_{\max }$ was recorded during maximum inspiratory effort at residual volume and $P E_{\max }$ was recorded during maximum expiratory effort from near total 
pulmonary capacity (Kanengiser \& Dozor, 1994). A three-way valve system with a mouthpiece (Air life 001504) was used to record PImax and PEmax. Like spirometry, instructions were modified and adequate time was given to make sure children understood the procedure. Unlike spirometry, we used a cylindrical mouthpiece with three circular ridges, which provided extra grip to avoid slipping the mouthpiece out during the forceful expiratory effort.

During $\mathrm{PE}_{\max }$ measurement, first, examiner demonstrated the procedure using his own mouthpiece and once they understood the procedure, we asked them to place the mouthpiece in his or her mouth and take big breath and blow as hard as possible. Participants were encouraged to give their best effort. The pressure meter (mouthpiece) had 1.5mm diameter leak to prevent glottis closure and reduce the contribution from buccal muscle during airway pressure measurement.

To measure $\mathrm{Pl}_{\max }$, after examiner demonstrated, participants were asked to put the mouthpiece in mouth and breath out (empty all your chest) all air and once you empty, take a deep breath in. A nose clip was used during both measurements. The mouthpiece was connected to a pressure transducer through a non-flexible tube. Participants were asked to maintain the pressure for at least two seconds and the highest value of each maneuver was used for statistical analysis. Testing was repeated until three similar attempts (within 10\% difference) were obtained (Tomalak, Pogorzelski, \& Prusak, 2002) (Sevda C. Aslan, Manpreet K. Chopra, William B. McKay, Rodney J. Folz, \& Alexander V. Ovechkin, 2013). 


\section{Surface Electromyography}

sEMG signals recorded during voluntary or involuntary movements provide information about motor control of muscles involved during those movements. This motor control is disrupted following SCl. Therefore, recording sEMG signals from both affected and unaffected muscles will characterize the impaired motor control after SCI (Lim et al., 2005; Sherwood, McKay, \& Dimitrijevic, 1996).

For this study, we recorded sEMG signals from the various trunk and respiratory muscles while subjects performed specific voluntary trunk and respiratory tasks were sitting (with assistance for $\mathrm{SCl}$ ) on the bench with their hip and knee joint flexed to 90 degrees.

Bipolar surface electrodes were used to record muscle activities from upper trapezius (UT), pectoralis major (PEC), external intercostal (INT), rectus abdominis (RA), external oblique (OB), thoracic paraspinal (PST) and lumbar paraspinal (PSL) during trunk and respiratory motor assessment (PEmax \& Plmax). Two ground electrodes, placed over shin of tibia were used as a reference point. Before placing the electrodes, the skin area above the muscle belly was cleaned by alcohol swabs and electrodes were secured by latex free and hypo allergic weaved tape (BSN medical) (S. C. Aslan et al., 2013). 


\section{Electrode Placements}

We recorded sEMG signals from seven muscles bilaterally. Electrodes for upper trapezius were placed just above the spine of scapulae at midclavicular line. Pectoralis major muscle electrodes were placed at an upper portion of pectoralis muscle at midclavicular line. External intercostal muscle electrodes were placed in $6^{\text {th }}$ intercostal space at the anterior axillary line. Electrode placement for rectus abdominus was at the midclavicular and the umbilical line. OB muscle electrodes were located at the maxillary line at umbilical level. Paraspinal muscle (PST and PSL) electrodes were placed $2 \mathrm{~cm}$ lateral to the spinous process of T9-T10 and L4-L5 vertebrae, respectively. The two ground electrodes were placed on the shin of tibia bilaterally. 


\section{Statistical Analysis}

All measurements (integrated sEMG, $\mathrm{PE}_{\max }, \mathrm{PI}_{\max }, \mathrm{FVC}, \mathrm{FEV}_{1}$, \& $\mathrm{FEV}_{1} / \mathrm{FVC}$ ) were presented in mean and standard deviation. Both trunk and respiratory motor control data were analyzed in $\mathrm{R}$ studio statistical software (RStudio, 2012). Data in bar plots are represented as the mean \pm SD. Criteria for outliers were defined and excluded at 3 SD from mean (Iglewicz B, 1993).

Normality tests indicated that integrated sEMG data (trunk and respiratory) was not normally distributed so, for each variable we assigned a log value and applied a general linear mixed model (GLMM).

For our first and second hypothesis, to compare TD preschool and school age children for trunk and respiratory motor outcomes (sEMG amplitude), GLMM test was used to test age main effect. For our third and fourth hypothesis, to compare trunk and respiratory variables between TD and children with SCI, multiple GLMM tests were used due to many variables related to the injury that could affect the functional outcomes in children with SCI. Therefore, children in SCI group were further grouped based on the age (preschool vs. school-age) and levels of SCI for both trunk motor control outcomes. 


\section{$\&+\$ 37(5,9$ TRUNK MOTOR CONTROL IN TYPICALLY ' ( 9 ( / 23,1 * CHILDREN}

\section{Introduction}

Our first Specific Aim was to establish normative, age dependent neurophysiological trunk motor control outcome measure in TD children. Studies in the past have assessed trunk as a single segment, but the trunk is made up of multiple spinal subunits, controlled by muscles at different levels. All the studies mentioned above have examined the sitting posture control in children until they attain independent sitting, standing or walking, but there is a lack of information about how posture control develops further with development after achieving these major milestones. Such missing information is crucial in understanding and comparing the atypical trunk control (sitting) as observed in children with neuromuscular diseases.

Poor trunk control is a characteristic feature among children, adolescent, and adults with cerebral palsy, which results in difficulty in performing ADLs, such as sitting and walking. However, children with neuromuscular diseases such as $\mathrm{SCl}$ also exhibit similar impairment in trunk control, which results in an inability to sit and non-ambulatory. This impairment in trunk control may cause compensatory movements in other segments of the body, which cause further 
deviation from the typical pattern (control). Therefore, assessment of trunk control in TD children in the seated position could provide valuable information, which may help to understand the impairment of trunk control in children with neuromuscular diseases.

Therefore, we studied changes in the trunk motor control within TD children in association with age (Preschool vs School age). We hypothesized that the neurophysiological characteristics of trunk motor control depend upon age: i.e. older children (6-13 years) would demonstrate higher sEMG amplitude than children in younger age group (3-5 years).

\section{Methods}

Trunk control was assessed using SATCo test. Participants were tested in seated position with hip and knee both at 90 degrees of flexion with feet on ground and back unsupported. Simultaneously sEMG signals from various trunk muscles (RA, OB, PST, and PSL) were recorded. Please refer to chapter III (page 49).

\section{Results}

The sEMG amplitude of trunk muscles were significantly higher in the preschool children compared to children in the school-age group (Figure IV.I). We found significant differences in sEMG amplitude between the two groups for all the different levels of SATCo test except, BRSC and NSSC levels. SSC $(p=.001)$, ASC $(p=.002)$, ISSC $(p=.01)$, OLRSC $(p=.04)$, and PSC $(p=.03)$ (Figure IV.I) with increased trunk muscle activation in preschool children. However, BRSC and NSSC levels were not significantly different between two groups 
$(p=.09)(p=11)$ respectively. Significant differences between two groups were found for RA $(p=.002)$ and PST $(p=.03)$ muscles activation with higher activation in the preschool group (Figure IV.II-VIII). 


\section{SATCo Levels}

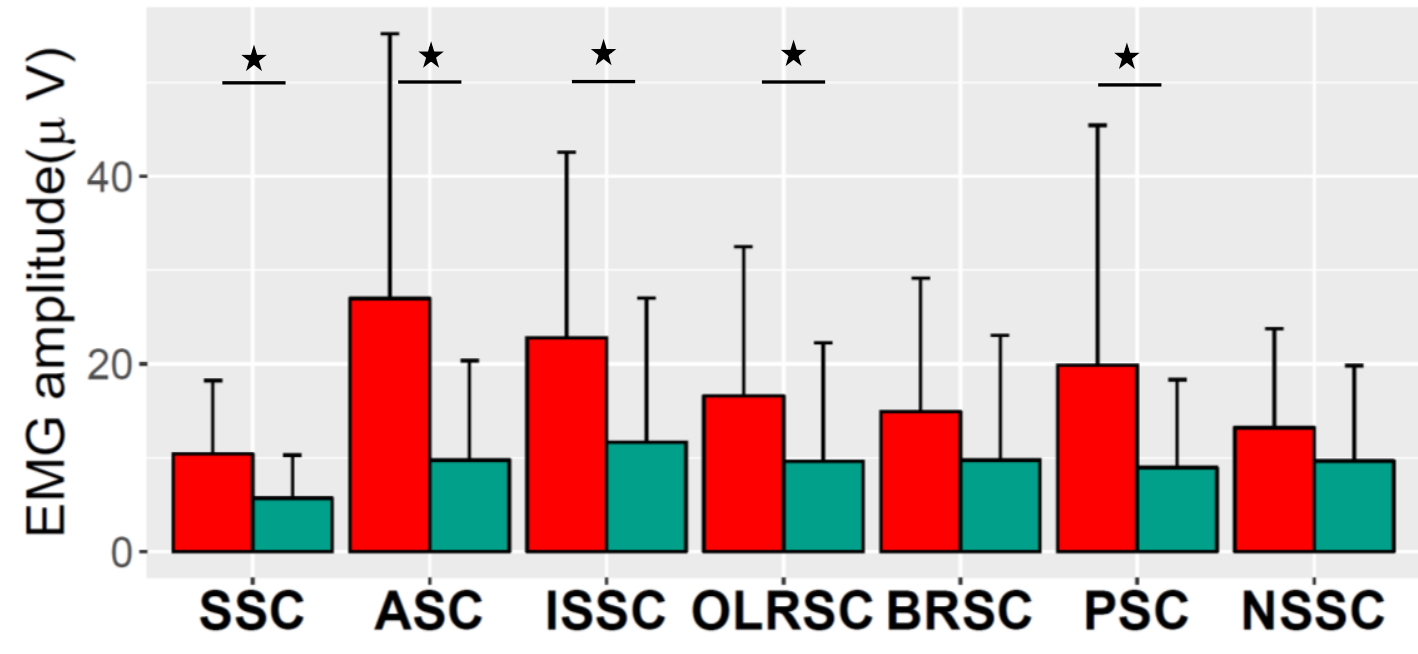

\section{AgeGroup —Preschool \$School-Age}

Figure IV I: Trunk muscle activation during SATCo test in TD children

Activation of the trunk muscles during the segmental assessment of trunk control (SATCo) testing. Overall activation of the trunk muscles, rectus abdominous, external oblique, thoracic paraspinal and lumbar paraspinal (microvolts) is plotted against different levels of trunk support during SATCo testing. Significant differences between preschool and school-age groups were found for shoulder static control level (SSC) ( $p=.001)$, axilla static control (ASC) $(p=.002)$, inferior scapula static control (ISSC) ( $p=.01)$, over lower ribs static control (OLRSC) $(p=.04)$, and pelvis static control (PSC) $(p=.03)$ with higher muscle activation in preschool children. However, no significant differences were found between two groups for below ribs (BRSC) ( $p=.09$ ), and no support static control levels (NSSC) $(p=.11)$. The values are represented as a mean \pm standard deviation. 


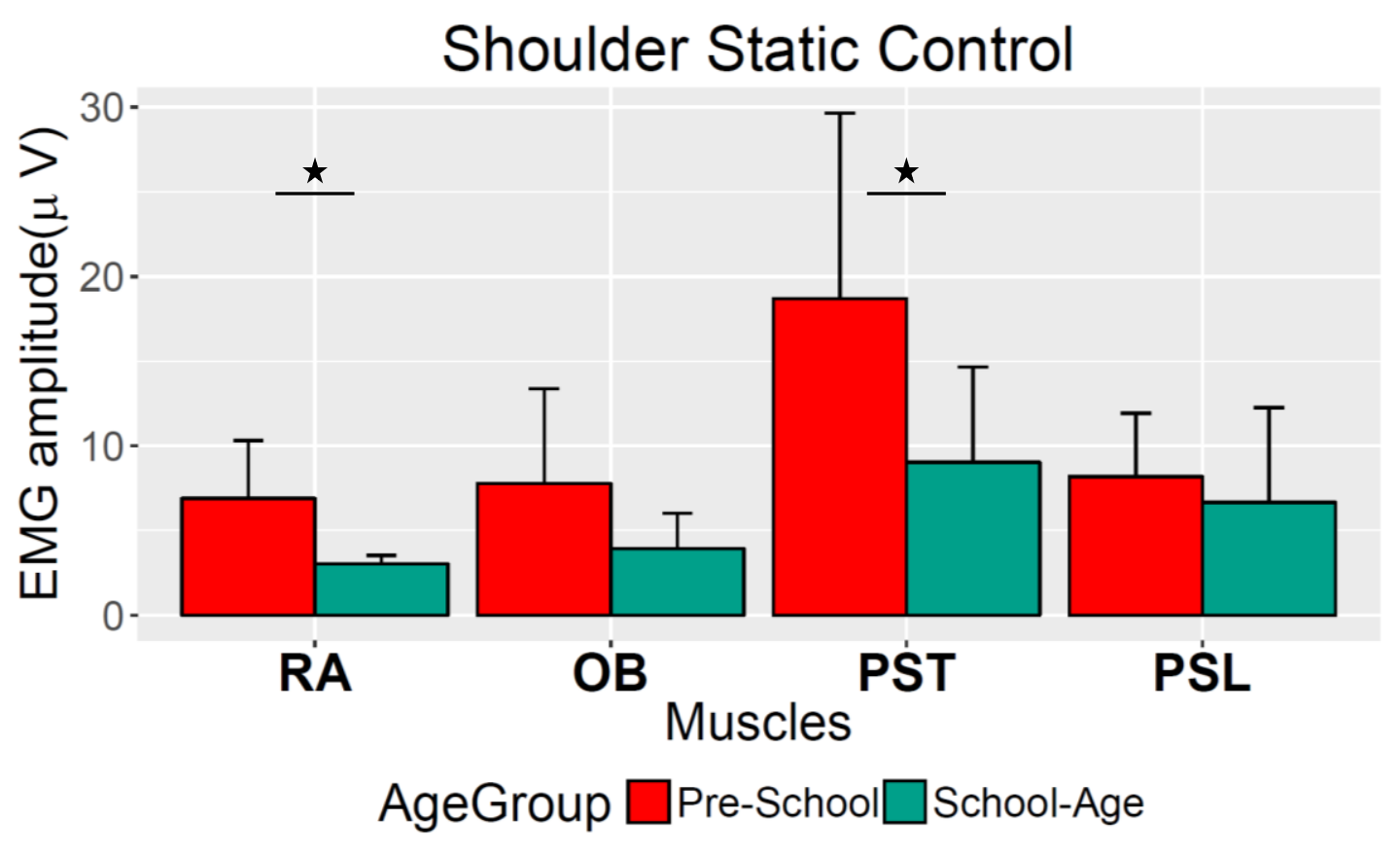

Figure IV:I: Trunk muscle activation during SSC in TD children

Electromyography amplitude from various trunk muscles calculated as the root mean square (RMS) values in microvolts is plotted on the $y$-axis. RMS values of rectus abdominis (RA), external oblique (OB), paraspinal at thoracic (PST) and lumbar levels (PSL) calculated during testing at shoulder static control level of segmental assessment of trunk control test (SATCo). Significant differences between two groups were found for RA ( $p=.002)$ and PST $(p=.03)$ muscles activation, with higher activation in the preschool group compared to school-age group. No significant differences were observed for OB $(p=.10)$ and PSL $(p=.32)$ muscles activation between preschool and school-age group. The values are represented as a mean \pm standard deviation. 


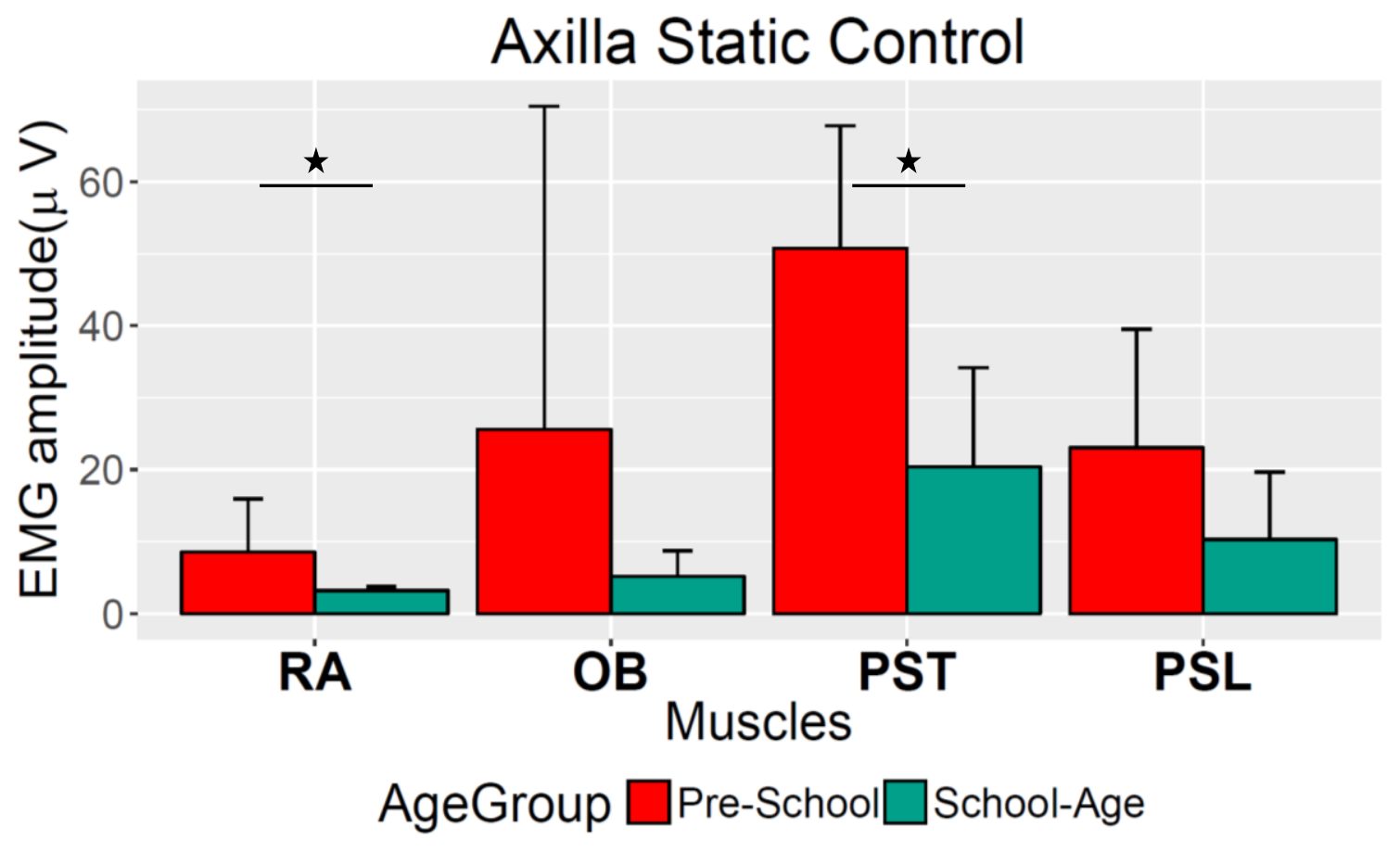

Figure IV:II: Trunk muscle activation during ASC in TD children

Electromyography amplitude from various trunk muscles calculated as the root mean square (RMS) values in microvolts is plotted on the $y$-axis. RMS values of rectus abdominis (RA), external oblique (OB), paraspinal at thoracic (PST) and lumbar levels (PSL) calculated during testing at Axilla static control level of segmental assessment of trunk control test (SATCo). Significant differences between two groups were found for RA ( $p=.006)$ and PST $(p=.01)$ muscles activation, with higher activation in the preschool children compared to children in school-age group. OB $(p=.18)$ and PSL $(p=.11)$ muscles activation between preschool and school-age groups were not significantly different. The values are represented as a mean \pm standard deviation. 


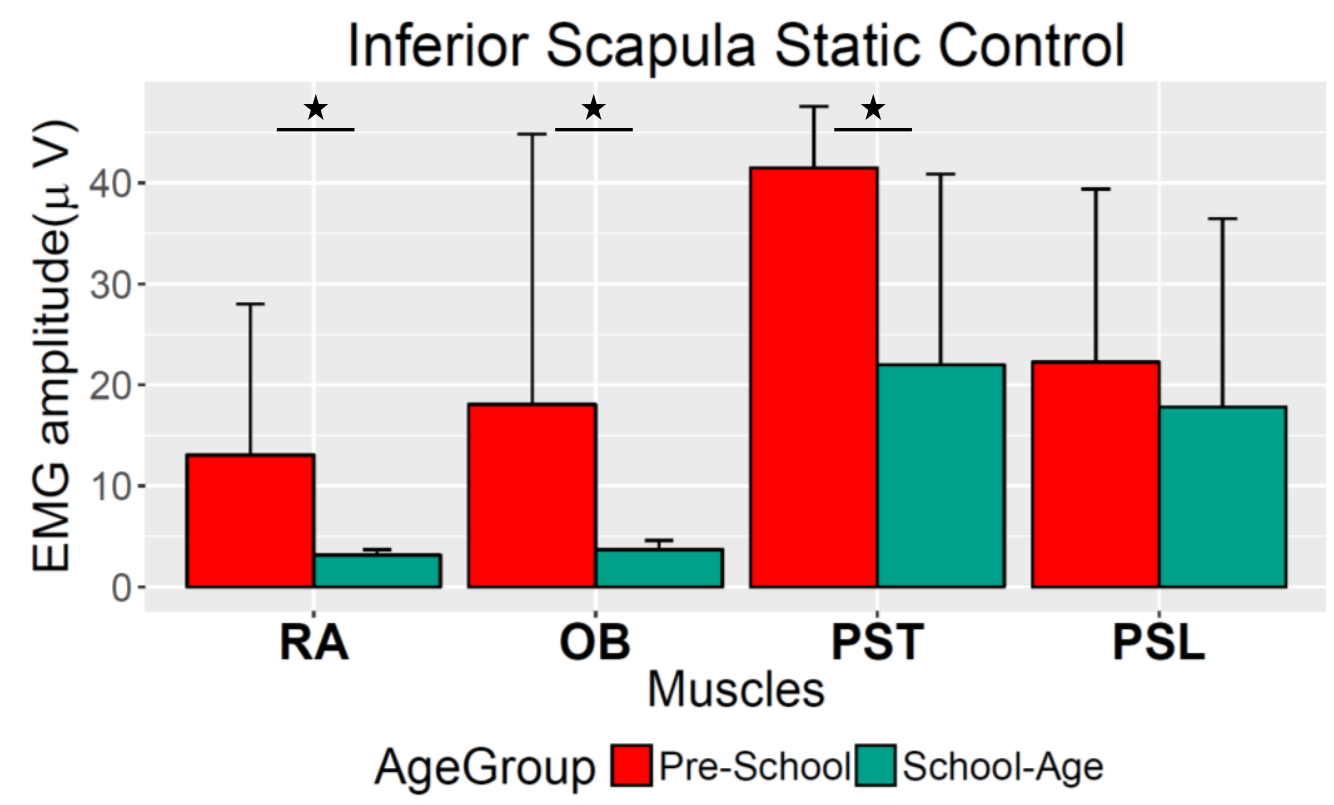

Figure IV:III: Trunk muscle activation during ISSC in TD children

Electromyography amplitude from various trunk muscles are plotted during trunk control testing at Inferior scapula level (ISSC). The sEMG amplitude of rectus abdominous (RA), external oblique (OB) and thoracic paraspinal muscles (PST) was significantly higher muscle activation in the preschool group than children in school-age group ( $p=.005),(p=.03),(p=.05)$ respectively. Lumbar paraspinal muscle (PSL) activation was not significantly different between the two groups $(p=.61)$. The values are represented as a mean \pm standard deviation. 


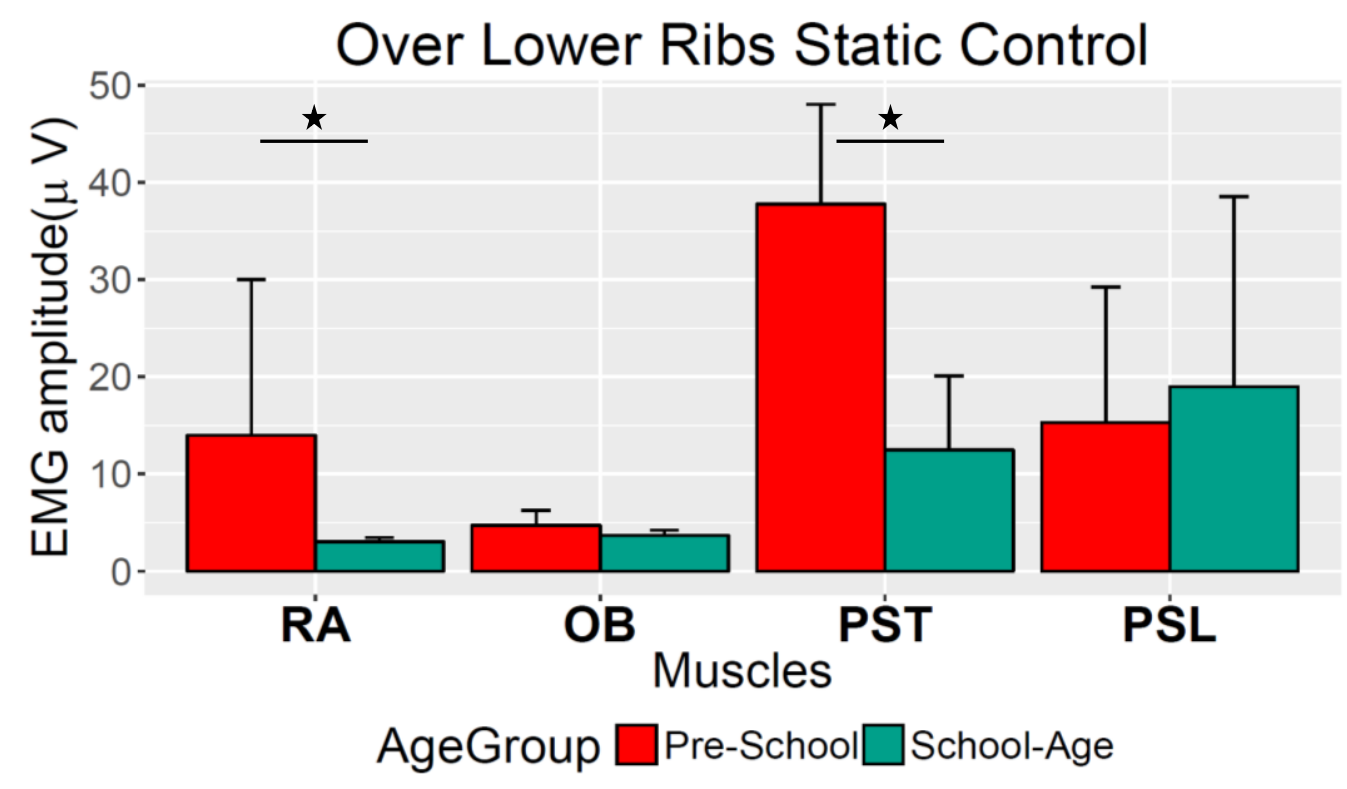

Figure IV:IV: Trunk muscle activation during OLRSC in TD children

Electromyography amplitude from various trunk muscles calculated as the root mean square (RMS) values in microvolts is plotted on the $y$-axis. RMS values of rectus abdominis (RA), external oblique (OB), paraspinal at thoracic (PST) and lumbar levels (PSL) calculated during testing at over lower ribs level of segmental assessment of trunk control test (SATCo). Children in the preschool group showed significantly higher activation in RA $(p=.006)$ and PST $(p=.006)$ muscles compared to children in school-age group. However, no significant differences were found between two groups for OB $(p=.09)$, and PSL $(p=.96)$ muscle activation. The values are represented as a mean \pm standard deviation. 


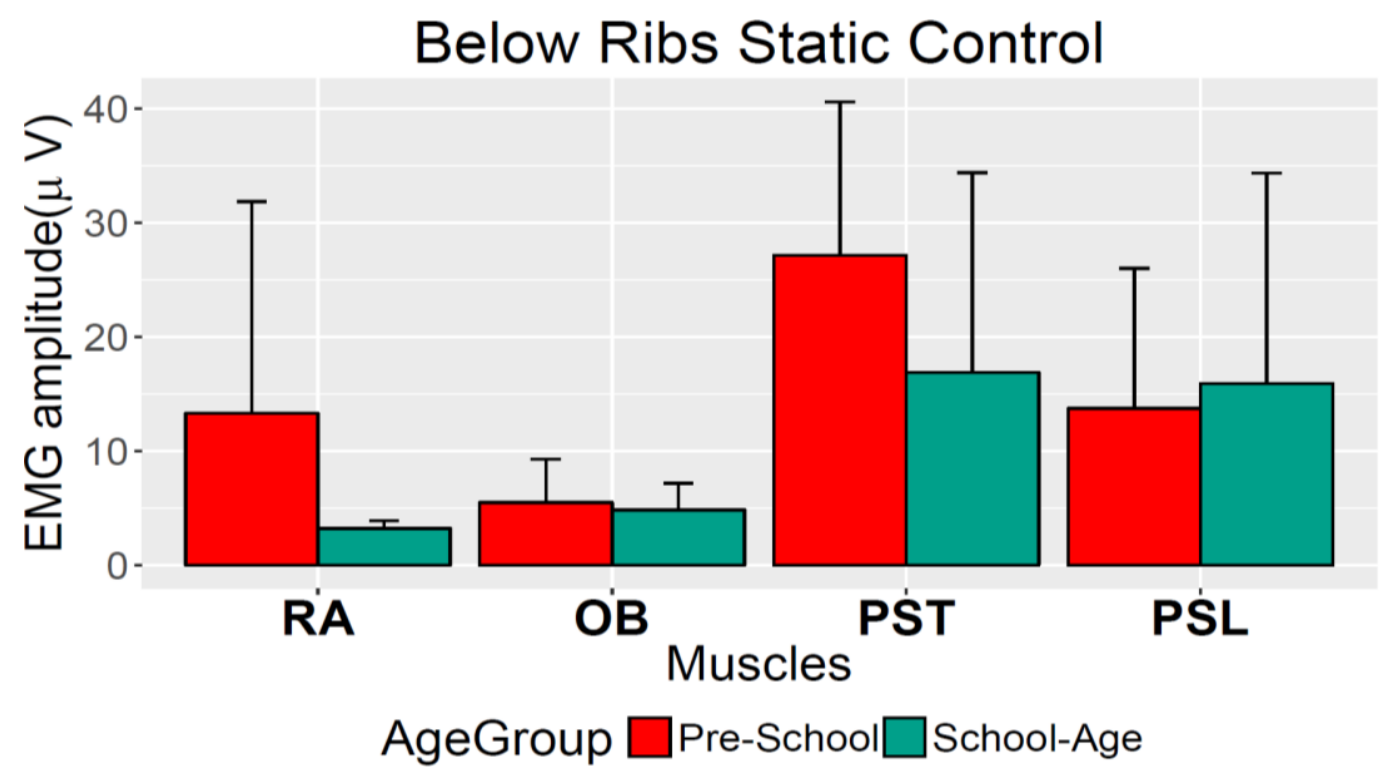

Figure IV:V: Trunk muscle activation during BRSC in TD children

Electromyography amplitude from various trunk muscles calculated as the root mean square (RMS) values in microvolts is plotted on the $y$-axis. RMS values of rectus abdominis (RA), external oblique (OB), paraspinal at thoracic (PST) and lumbar levels (PSL) calculated during testing at below ribs level of segmental assessment of trunk control test (SATCo). No significant differences between the two groups were observed for RA ( $p=.07)$, PST $(p=.12)$, OB $(p=.95)$ and PSL $(p=.74)$ muscles activation. The values are represented as a mean \pm standard deviation. 


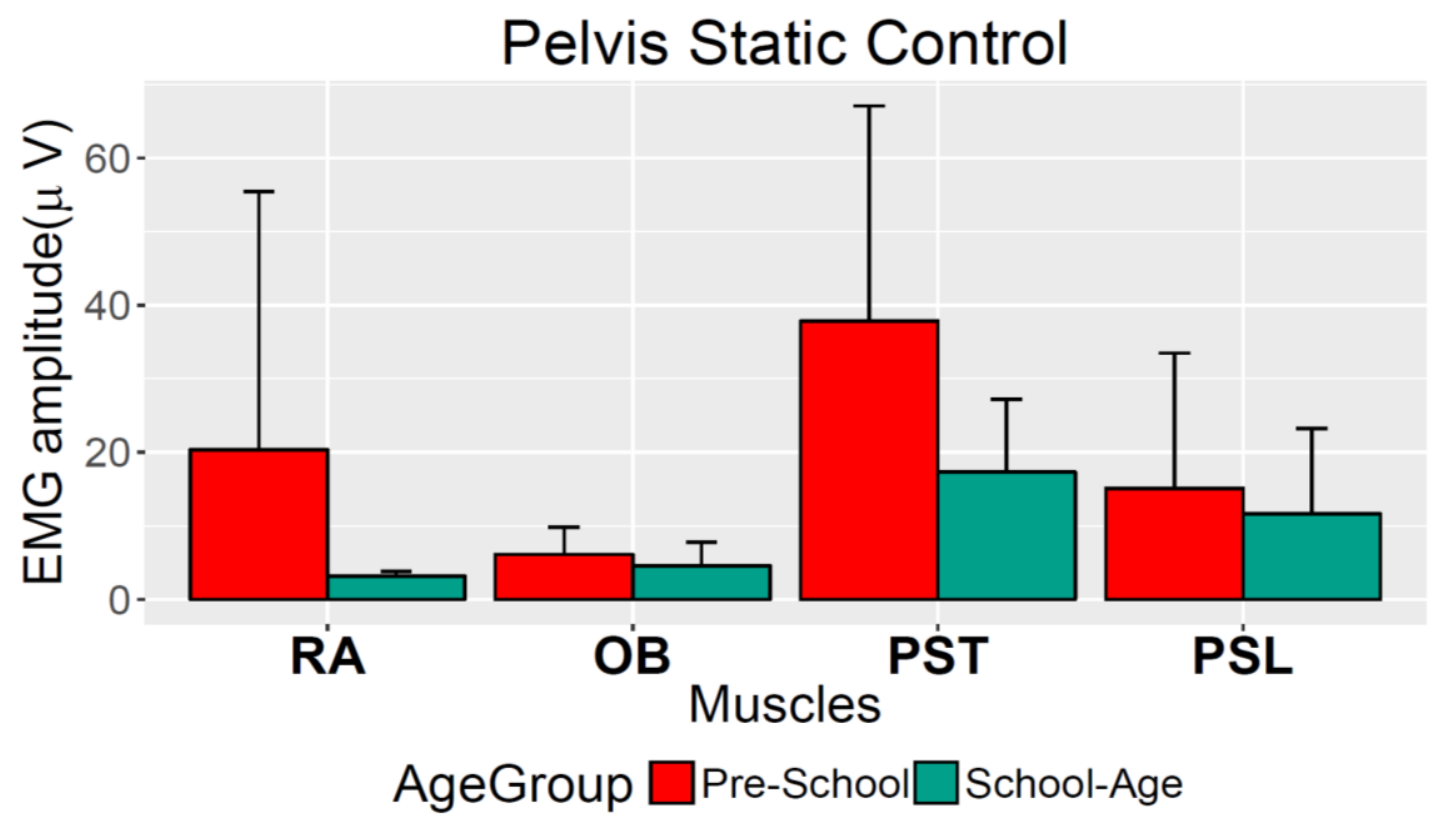

Figure IV:VI: Trunk muscle activation during PSC in TD children

Electromyography amplitude from various trunk muscles calculated as the root mean square (RMS) values in microvolts is plotted on the $y$-axis. RMS values of rectus abdominis (RA), external oblique (OB), paraspinal at thoracic (PST) and lumbar levels (PSL) calculated during testing with support at the pelvic level. No significant differences were observed for RA ( $p=.36)$, PST ( $p=.08)$, OB $(p=.40)$ and PSL $(p=.65)$ muscles activation. The values are represented as a mean \pm standard deviation. 


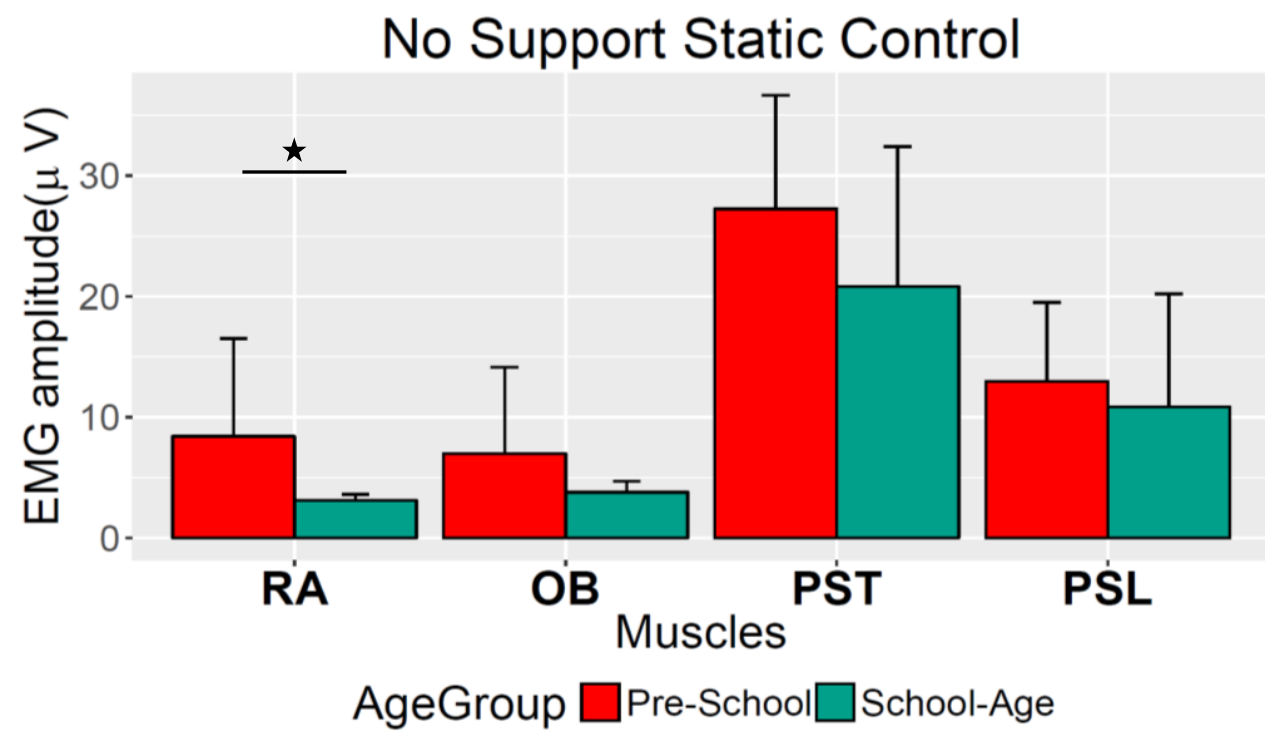

Figure IV:VII: Trunk muscle activation during NSSC in TD children

Electromyography amplitude from various trunk muscles calculated as the root mean square (RMS) values in microvolts is plotted on the $y$-axis. RMS values of rectus abdominis (RA), external oblique (OB), paraspinal at thoracic (PST) and lumbar levels (PSL) calculated during testing with no support. Muscle activation of RA ( $p=.02$ ) was significantly higher in preschool children compared to children in the school-age group. However, no significant differences were recorded for PST $(p=.27)$, OB $(p=.19)$ and PSL $(p=.41)$ muscle activation between the two groups. The values are represented as a mean \pm standard deviation. 


\section{Discussion}

The aim of our study was to establish normative, age-dependent (3-13 years) neurophysiological trunk motor control outcome measures in neurologically intact healthy children. We first examined integrated sEMG signals from different trunk muscles during trunk control assessment using SATCo test between preschool and school-age children. We found that children in the preschool group showed higher activation of trunk muscles for all the levels of support (Figure IV.I). However, while comparing the sEMG measure, the overall pattern of muscle activation showed a correlation between sEMG responses and age, with younger children (preschool) exhibiting higher trunk muscle activity at sitting upright position than children in older group (school-age).

Activation of the lower trunk muscles in both, preschool and school age children indicates the muscle activation necessary to maintain spine stability in a neutral position, suggesting that activation of the trunk muscles is critical in providing mechanical stability to the spine during sitting upright position. However, children in school-age group produced lower activation in trunk muscles compared to children in preschool group with less variability and minimal energy expenditure.

According to neuronal group selection theory, variation is motor behavior is the principle property of normal development (Hadders-Algra, 2000, 2010). During the development process, infants present with abundant variation in motor behavior due to the availability of wide range of motor options. As development 
continues, more goal-directed movements then gradually replace this variation in motor response for a specific task with reduced variability. Therefore, with development, children start to use more efficient motor strategies with increased control of movements when tested for a specific motor task. Similarly, in this study, children in older age group produced more refined and efficient muscle activations when tested for these specific motor tasks. We also reported high variability in trunk muscle activation in preschool children, whereas children in school-age group showed less variability in trunk muscle activation during different SATCo level, suggesting children gradually learns to control the degrees of freedom involved in head and trunk control during development as they start to develop adults like patterns of muscle activation.

Our results are consistent with studies in adults, where they have demonstrated similar patterns of activation of trunk muscles in the seated position. Children in school age group showed consistently, adult like muscle activation patterns with reduced agonist and antagonist contraction in seated upright position (J. Cholewicki, Panjabi, \& Khachatryan, 1997; McGill, Grenier, Kavcic, \& Cholewicki, 2003). In contrast to school age group, children in preschool age group showed more variable motor responses with increased activation of agonist and antagonist muscles (Figure IV.I-VIII). However, the muscle (postural) response through different levels was consistent and organized for all the different levels of support. 
Activation of trunk muscles is necessary for the maintaining an upright sitting and standing posture. However, sensory information about the position and movement of the body in space is also necessary for adequate posture control. CNS receives and interprets the information from vision, vestibular, and somatosensory receptors. Number of investigators have studied development of sensory adaption in children and result of combined studies suggest that children from 1.5 to 3 years old sway more than older children and adults with intact vision, vestibular and somatosensory systems (Ferber-Viart, lonescu, Morlet, Froehlich, \& Dubreuil, 2007; H Forssberg \& Nashner, 1982; Foudriat, Di Fabio, \& Anderson, 1993). Therefore, sensory system plays an important role in posture control. Younger children in our study might have less improved adaption to sensory systems, which resulted in more variability (higher activation) in trunk muscle activation than compared to children in older age group.

Significant differences in the activation of trunk muscles between preschool and school age children address the question that, younger children (preschool) are lacking organized patterns of muscle activity with greater variability in their motor response compared to older children who use efficient co-contraction of trunk muscles with reduced variability. This could be due to gradual and efficient control of degrees of freedom of head and trunk by children in older group. These results suggest that sitting posture control is a dynamic process involving gradual control of degrees of freedom of head and trunk by activation of appropriate co-contraction of trunk muscles. 


\title{
$\&+\$ 37(59$ RESPIRATORY MOTOR CONTROL IN TYPICALLY ' $(9(/ 23,1 *$ CHILDREN
}

\author{
Introduction
}

\begin{abstract}
Assessment of pulmonary function is not only a fundamental tool in understanding the physiology of respiratory system, but also indispensable in the clinic to diagnose and manage respiratory diseases (Beydon et al., 2007).

Routine testing of lung functions and respiratory muscle testing are recommended in children with neuromuscular diseases (Fauroux, Quijano-Roy, Desguerre, \& Khirani, 2015). Current tools available to test respiratory functions in TD children are not designed to provide a quantitative evaluation of respiratory motor control. Standard measures like, Forced Vital Capacity (FVC), Forced Expiratory Volume in 1s (FEV 1$)$, Maximal Expiratory Pressure $\left(P E_{\max }\right)$ and Maximum Inspiratory Pressure $\left(\mathrm{PI}_{\max }\right)$ have been used to measure the strength of respiratory muscles (Jain, Brown, Tun, Gagnon, \& Garshick, 2006). However, standard pulmonary testing does not evaluate the multi-muscle motor control by the CNS.
\end{abstract}


Evaluation of multi-muscle motor control using surface electromyography can be used to measure the strength and diagnose any underlying neuromuscular pathology (American Thoracic Society/European Respiratory, 2002; S. C. Aslan et al., 2013). However, this multi-muscle motor control measurement along with standard pulmonary testing has not been tested in TD children. Therefore, our aim is to establish normative, age-dependent (3-10 years) neurophysiological respiratory motor control outcomes in TD children using this multi-muscle motor control model. We hypothesized that children in older age group produce higher lung volumes and airway pressures associated with higher sEMG amplitude than children in the younger group.

\section{Methods}

A total of 14 TD children completed the respiratory motor control testing with 5 children in preschool and 9 children in the school-age group. The mean age of children in preschool and the school-age group was $4(4 \pm .7)$ and $8(8 \pm 2)$ respectively. Demographics and respiratory measurements of children in preschool and school age group are listed in table V. I and V.II, respectively. First, children in both groups were asked to perform standard pulmonary function testing (FVC, FEV 1 ). After spirometry, subjects were tested for maximum expiratory $\left(P E_{\max }\right)$ and inspiratory airway pressure $\left(P I_{\max }\right)$ maneuvers while we simultaneously recorded surface electromyography from upper trapezius, pectoralis major, external intercostal, rectus abdominous, external oblique, paraspinal at thoracic and lumbar region. 


\section{Results}

Pulmonary function outcomes were significantly lower in the preschool children compared to children in the school age group: $\mathrm{FVC}, \mathrm{FEV}_{1}, \mathrm{PE}_{\max }$ and $\mathrm{Pl}_{\max }$ (Figure V.I-VI). Children in the school age group produced higher values of $\mathrm{FVC}$ and $\mathrm{FEV}_{1}$ than children in the preschool group. There was a strong, positive and linear $(r>.90)$ relationship between FVC and age for both preschool and school-age children. In addition, there was a strong, positive and linear $(r>.80)$ relationship between $\mathrm{FEV}_{1}$ and age for children in the school age group and preschool group (Figure VI.1-2). However, no significant differences were found between the two groups for respiratory muscle activation during $P E_{\max }$ and $P I_{\max }$ maneuvers (Figure V. VB \& VIB). 
Table V-I: Preschool-Typically Developing Children for Respiratory Motor Control Assessment

\begin{tabular}{|c|c|c|c|c|c|c|c|c|c|c|}
\hline $\begin{array}{c}\text { Subject } \\
\text { (ID) }\end{array}$ & $\begin{array}{c}\text { Age } \\
\text { (years) }\end{array}$ & Gender & $\begin{array}{l}\text { Height } \\
\text { (cm) }\end{array}$ & $\begin{array}{l}\text { Weight } \\
\text { (Kgs) }\end{array}$ & $\begin{array}{c}\mathrm{PE}_{\max } \\
(\mathrm{cmH20})\end{array}$ & $\begin{array}{c}\mathrm{PI}_{\max } \\
(\mathrm{cmH} 20)\end{array}$ & $\begin{array}{c}\text { FVC } \\
\text { (Liters) }\end{array}$ & $\begin{array}{c}\text { FEV }_{1} \\
\text { (Liters) }\end{array}$ & FVC\%P & $\mathrm{FEV}_{1} \% \mathrm{P}$ \\
\hline N149 & 3 & $F$ & 89 & 17 & 19 & -18 & 0.63 & 0.57 & 482 & 212 \\
\hline N150 & 4 & $M$ & 114 & 20 & 42 & -24 & 0.77 & 0.71 & 95 & 103 \\
\hline N133 & 4 & $M$ & 98 & 17 & 33 & NA & 0.84 & 0.84 & 64 & 69 \\
\hline N130 & 5 & $F$ & 114 & 20 & 53 & -45 & 1.4 & 1.3 & 126 & 122 \\
\hline N134 & 5 & $M$ & 106 & 17 & 66 & -44 & 1.2 & 1.1 & 126 & 126 \\
\hline Mean \&SD & $4 \pm 0.7$ & $2 F, 3 M$ & $104 \pm 9$ & $18 \pm 1$ & $42 \pm 16$ & $-32 \pm 11$ & $0.9 \pm 0.2$ & $0.9 \pm 0.2$ & $178 \pm 153$ & $126 \pm 47$ \\
\hline
\end{tabular}


Table V-II: School-age- Typically Developing Children for Respiratory Motor Control Assessment






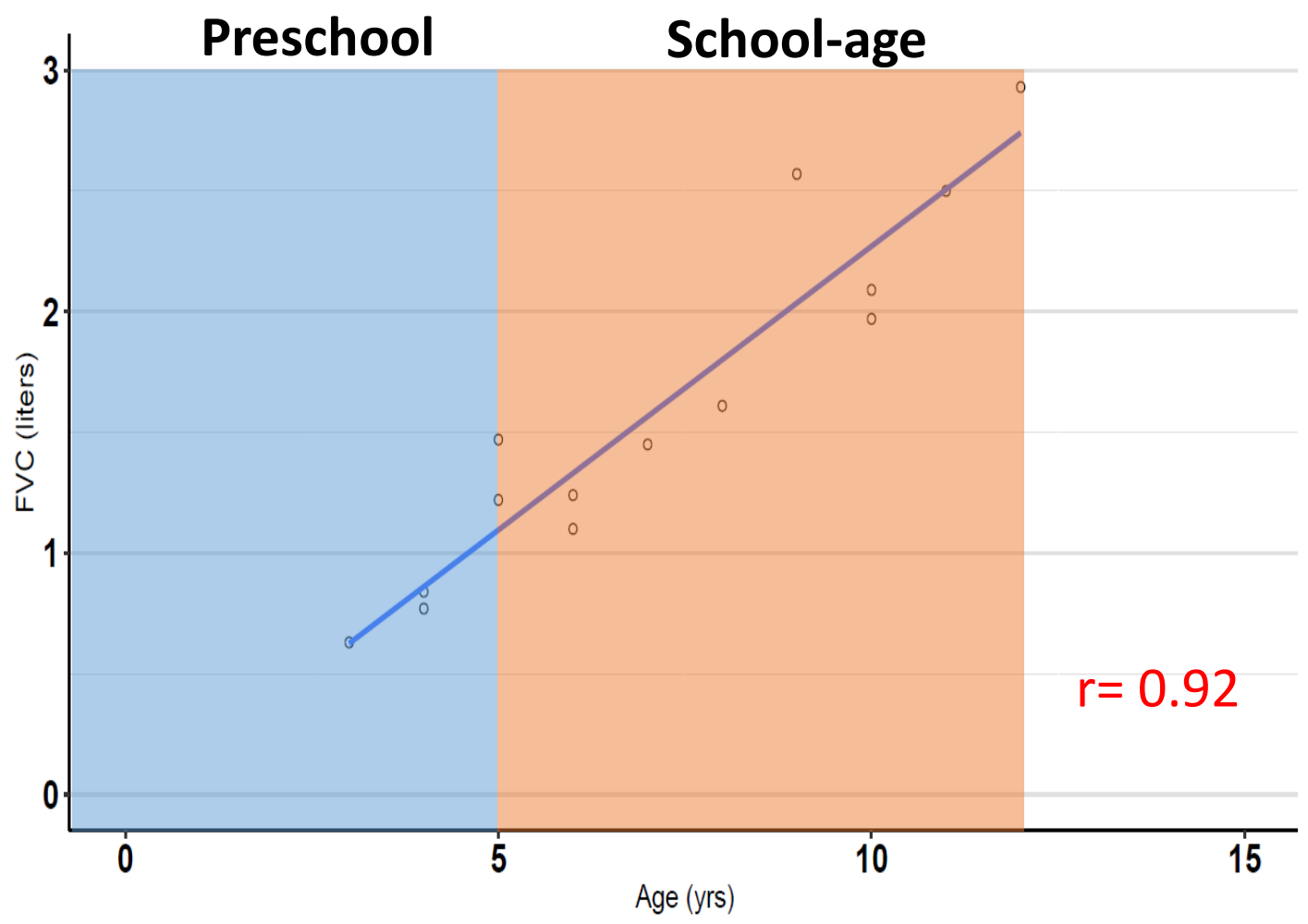

Figure V:I: FVC in TD children

Correlation between Forced Vital Capacity (liters) and age (years) between preschool and school-age groups. There is a strong, positive and linear relationship between FVC and age for children in TD group ( $r=.92)$ 


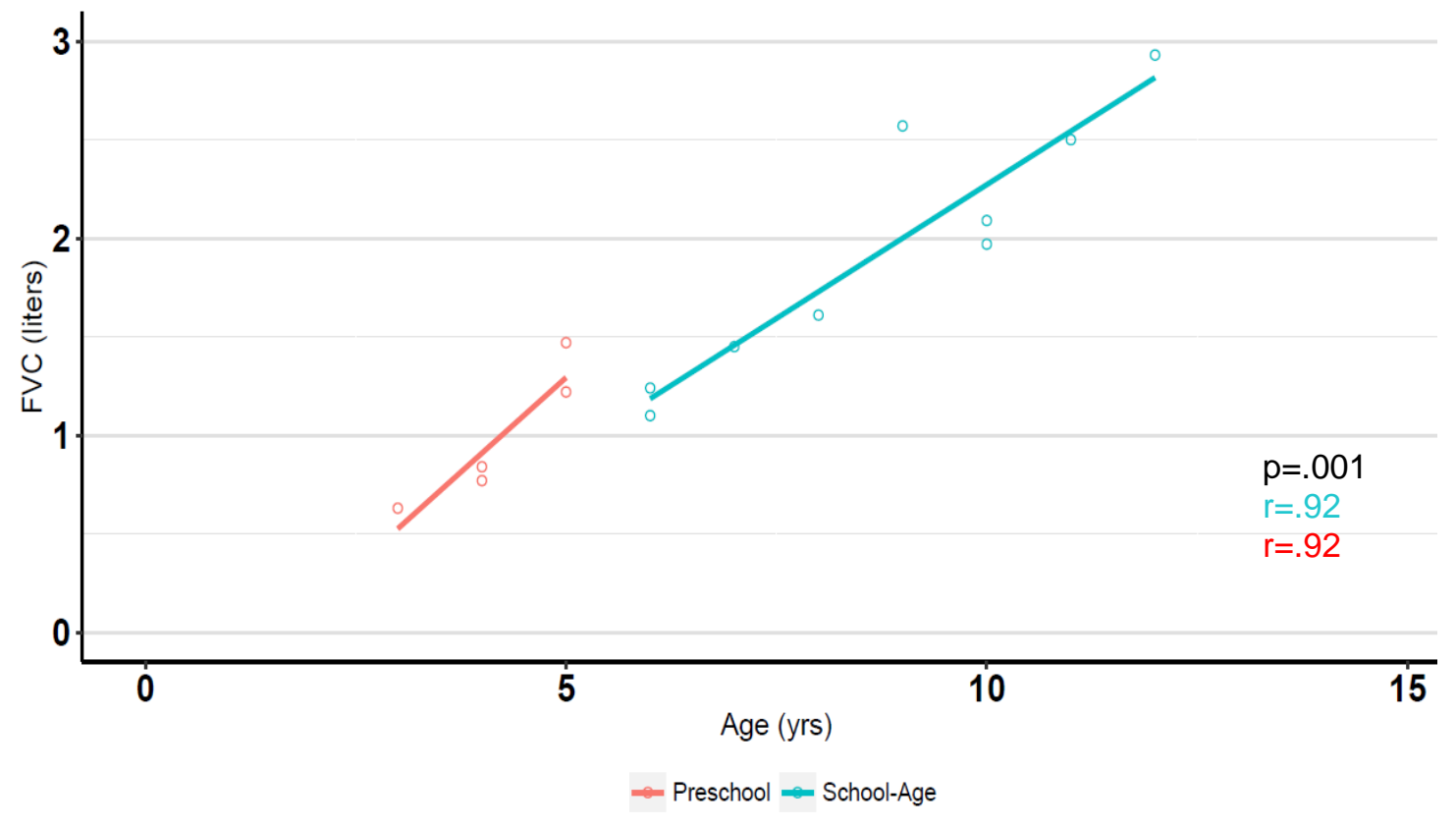

Figure V:II: FVC in preschool and school-age children

Correlation between Forced Vital Capacity (liters) and age (years) between preschool and school-age groups. A significant difference was observed between the two groups $(p=.0001)$. Children in school age group produced a greater volume of air (FVC) than children in preschool age. There was a linear relationship between FVC and age for children in both the preschool ( $r=.92)$ and school age children ( $r=.92)$. 


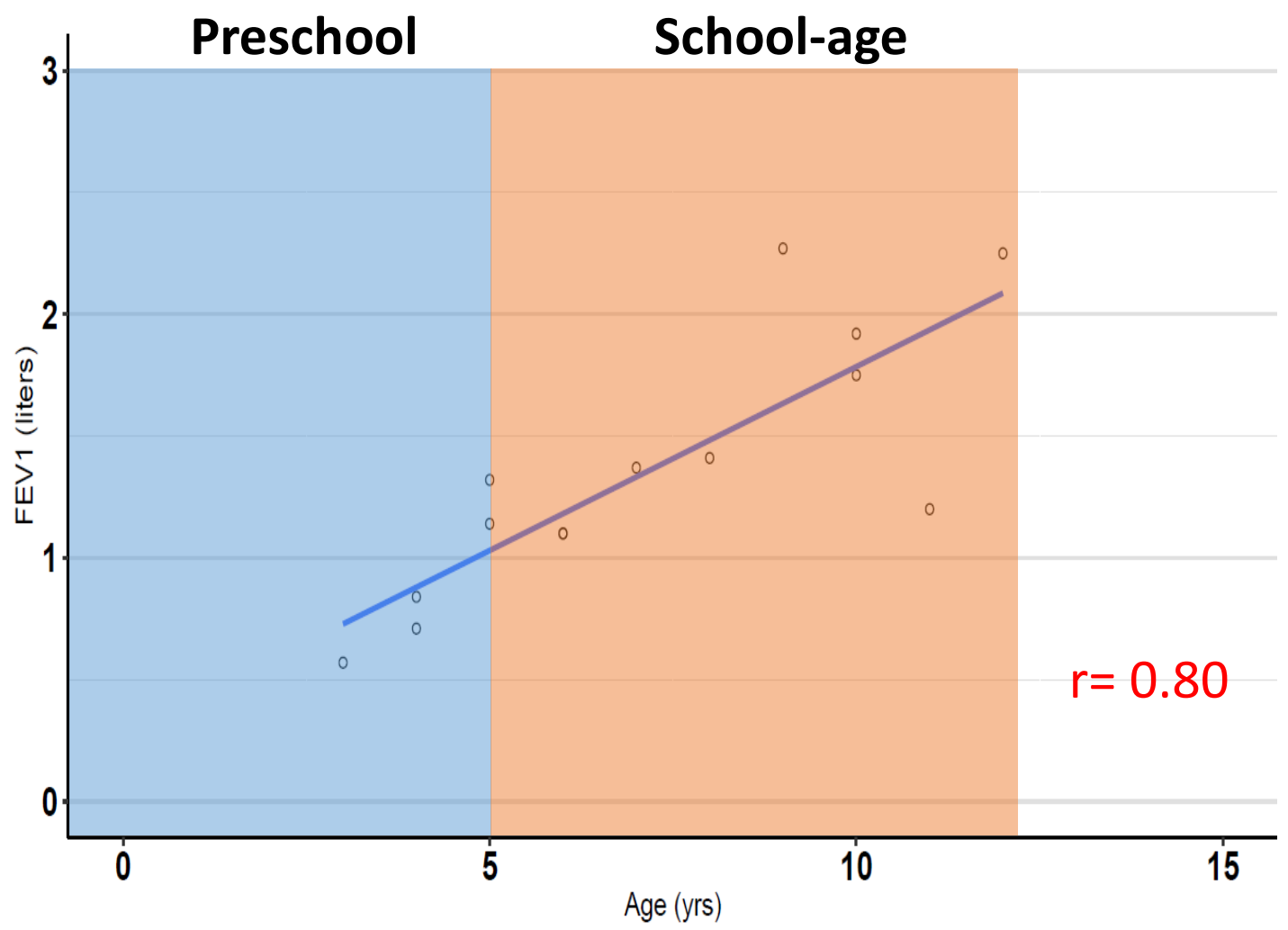

Figure V:III: $F E V_{1}$ in TD children

Correlation between Forced Vital Capacity (liters) and age (years) between preschool and school-age groups. There was a strong, positive and linear relationship between FVC and age for children in TD group $(r=.80)$ 


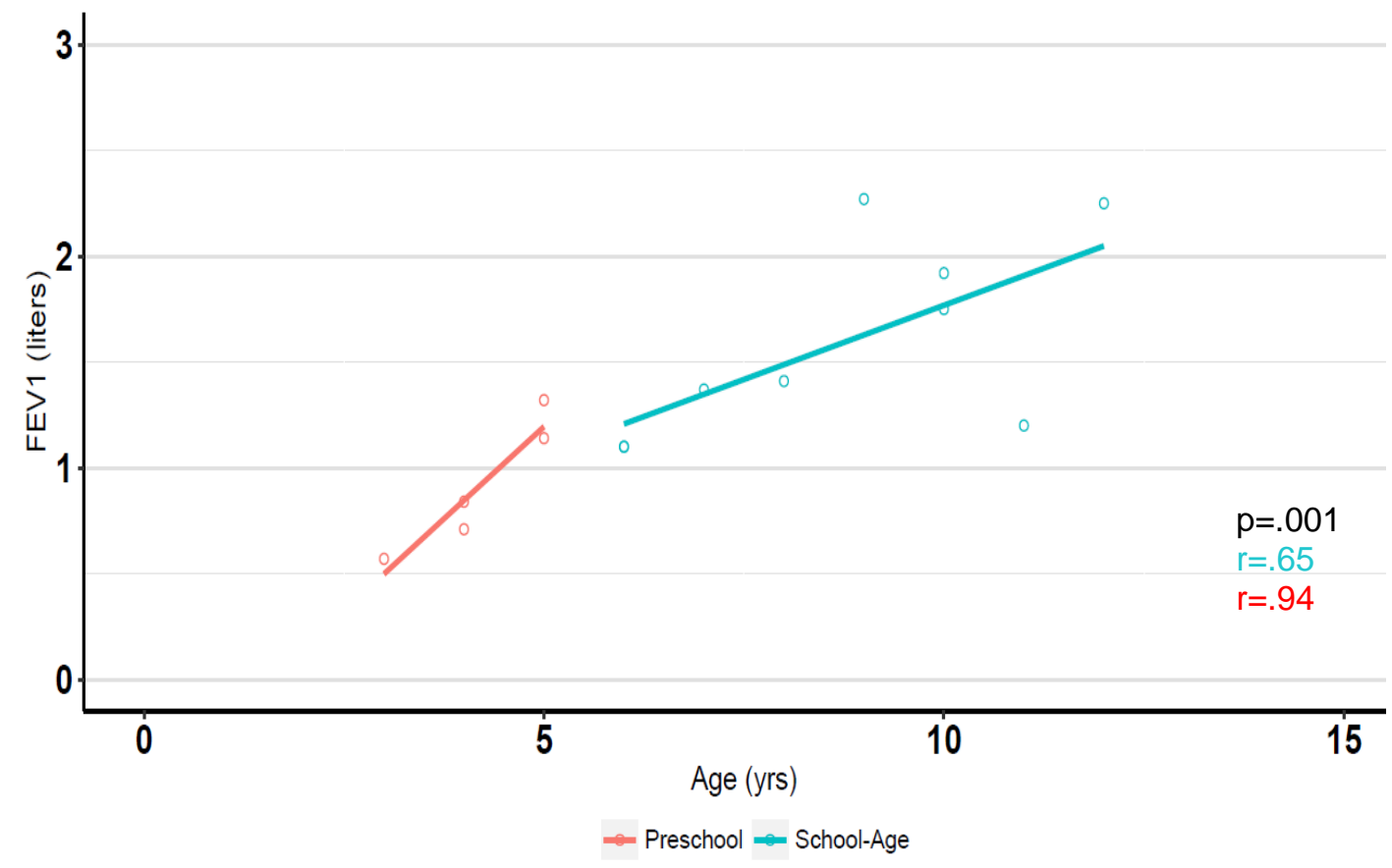

Figure V:IV: $F E V_{1}$ in preschool and school-age children

Correlation between Forced Expiratory Volume in one second (liters) and age (years) between preschool and school-age groups. A significant difference was observed between the two groups $(p=.0001)$. Children in school age group produced a greater volume of air in one second $\left(\mathrm{FEV}_{1}\right)$ than children in preschool age. There was a strong linear relationship between $\mathrm{FEV}_{1}$ and age for both children in the school age group ( $r=.65)$ and children in the preschool group $(r=.94)$. 

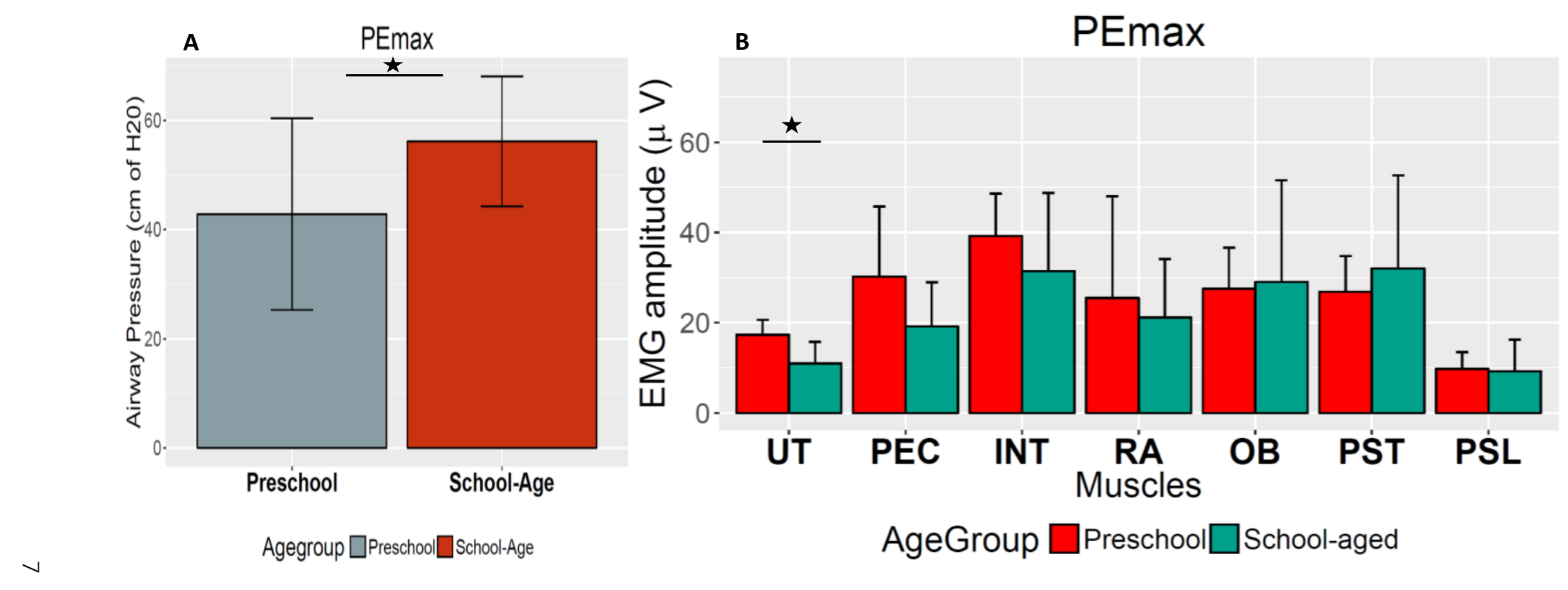

Figure V:V: PEmax and muscle activation in TD

A. Maximum expiratory airway pressure $\left(\mathrm{PE}_{\max }\right)$ between preschool and school-age groups. Children in preschool group produced significantly lower $(p=.0001)$ PEmax airway pressure when compared to children in the school-age group. B. Electromyography amplitude of respiratory muscles during the expiratory phase of PEmax between preschool and school age groups. Upper trapezius (UT) muscle activity was significantly lower in $(p=.04)$ preschool group than the school-age group. No significant differences were observed for external intercostal (INT) ( $p=.42)$, 
external oblique (OB) $(p=.66)$, pectoralis major (PEC) $(p=.21)$, lumbar paraspinal (PSL) $(p=.56)$, thoracic paraspinal (PST) ( $p=.99)$, and rectus abdominous (RA) ( $p=.63$ ) muscle activation. 

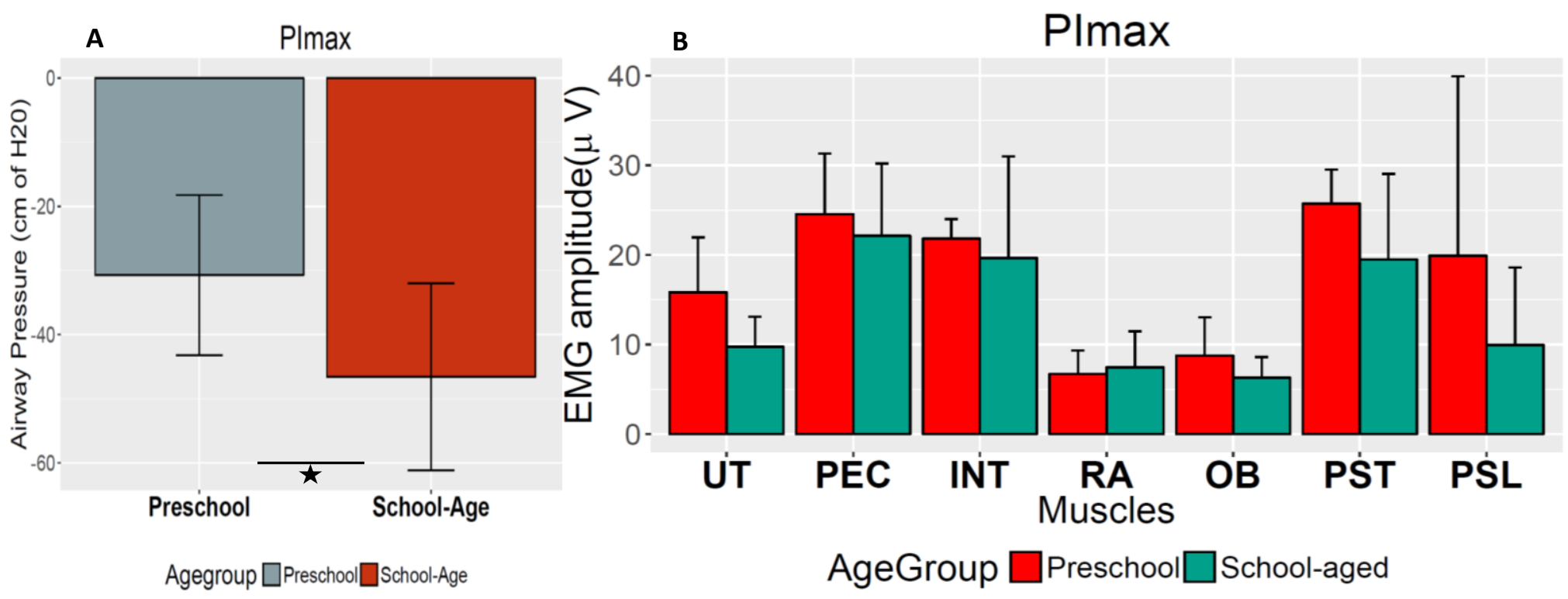

Figure V:VI: PImax and muscle activation in TD

A. Maximum inspiratory airway pressure $\left(\mathrm{PI}_{\max }\right)$ between preschool and school-age groups. Children in school-age group produced significantly higher ( $\mathrm{p}=$.0001) $\mathrm{PI}$ max airway pressure compared to children in the preschool group.

B. Electromyography amplitude of respiratory muscles during the inspiratory phase of $\mathrm{PI}$ max between preschool and school age groups. No significant differences were observed for upper trapezius (UT) ( $p=.07$ ), pectoralis major (PEC) ( $p=.55)$, external intercostal (INT) $(p=.43)$, paraspinal at thoracic level (PST) $(p=.17)$, paraspinal at lumbar level (PSL) $(p=.23)$, rectus abdominis (RA) $(p=.79)$ and external oblique $(\mathrm{OB})(p=.35)$ muscles activation. 


\section{Discussion}

For this part of the study, we measured FVC, FEV $1, \mathrm{PE}_{\max }, \mathrm{PI}_{\max }$ and sEMG amplitude during $P E_{\max }$ and $P \mathrm{I}_{\max }$ maneuvers in TD children between the ages of 3-13 years. We found that our results were consistent with our hypothesis. Spirometry and airway pressure generation (PEmax \& $\mathrm{PI} \max )$ were significantly higher among school-age children compared to children in preschool group. However, sEMG amplitudes of various respiratory muscles for both, PEmax \& $\mathrm{Pl}_{\max }$ did not show significant differences between the two groups. The mean values of $F V C$ and $F E V_{1}$ in school-age children were $1.9 \pm .6$ and $1.5 \pm .4$ liters, respectively.

Children in preschool age group had FVC and $\mathrm{FEV}_{1}$ mean values of $0.98 \pm .3$ and $0.90 \pm .2$ liters respectively. In the preschool group, the mean $P E_{\max }$ and $\mathrm{PI}_{\max }$ values were $42 \pm 16$ and $-32 \pm 11 \mathrm{~cm} \mathrm{H} \mathrm{H}_{2} \mathrm{O}$, respectively. Whereas PEmax and PImax values in school age groups were $54 \pm 14$ and $-50 \pm 11 \mathrm{~cm} \mathrm{H} \mathrm{H}_{2} \mathrm{O}$ respectively. Results of $\mathrm{FVC}, \mathrm{FEV}_{1}, \mathrm{PE}_{\max }$ and $\mathrm{PI}_{\max }$ demonstrate a positive correlation between age and inspiratory, expiratory muscle strength as well as ventilation; children in school-age group produced greater airway pressure and FVC \& FEV 1 volumes.

Spirometry $\left(F V C, F E V_{1}\right)$ in clinics is used as a screening test to evaluate symptoms or signs of restrictive or obstructive lung diseases. In the case of respiratory disorders, it is used to assess prognosis or to monitor therapeutic intervention (Miller et al., 2005). 
Higher values of $\mathrm{FVC}, \mathrm{FEV}_{1}, \mathrm{PE}_{\max }$ and $\mathrm{PI}_{\max }$ in school age children represent the considerable growth and development of the respiratory system that occurs, with associated changes in lung mechanics. During PEmax maneuver, both external oblique and rectus abdominis muscles showed increased activation in both groups. However, no significant differences in muscle activation were observed between the two groups. Both these muscles play an important role in raising the intra-abdominal pressure.

During forced expiratory maneuvers (PEmax \& cough), the combined contraction of abdominal muscles (external oblique, rectus abdominis), increase intra-abdominal pressure by displacing diaphragm cranially. This action enables the cranial movement of air in the lungs and results in efficient forced expiration (Cresswell, GrundstrÖM, \& Thorstensson, 1992; Ito et al., 2016). Upper trapezius and intercostal (inspiratory) muscles activation was in both preschool and schoolage group during $\mathrm{Pl}_{\max }$ maneuver. These muscles help to elevate the chest cranially and laterally to the capacity of lungs (T. A. Wilson, Legrand, Gevenois, \& De Troyer, 2001).

Children in both groups produced increased activation in these muscles, but no significant differences were reported. Therefore, due to intact innervation, children in both groups activated of all the respiratory muscles needed during these maneuvers. Children in both groups, preschool and school-age demonstrated a significant relationship between age and FVC and FEV ${ }_{1}$. This indicates a positive correlation between growth and pulmonary outcomes. These 
results are expected because of significant growth and development changes in TD children.

Spirometry measurements are frequently used in older children but rarely measured in preschool children due to the notion that they are unable to perform a valid forced spirometer maneuver. This study also confirmed that valid spirometry curves can be obtained in preschool children, suggesting its feasibility in both, younger and older children. 


\section{$\&+\$ 37$ ( 59 , TRUNK MOTOR CONTROL IN CHILDREN WITH SPINAL \&25' INJURY \\ ,ntroduction}

Injury to the spinal cord can lead to paralysis, paresis or spasticity of muscles at and below the lesion site. A major proportion of children and adults with $\mathrm{SCl}$ perform most of their ADLs in seated position. Independent sitting is a major milestone and is also a prerequisite for optimal performance of ADLs. Compare to standing and walking, sitting posture takes the relatively larger base of support, but it still requires adequate posture control of the trunk and head. During typical development, posture control of trunk is different for different trunk segments (Curtis et al., 2015; Rachwani et al., 2013; Sandra L. Saavedra, 2012). However, this balance while sitting is impaired in $\mathrm{SCl}$ subjects due to sensorimotor deficits, which results in an inability to sit without support. This impairment in the trunk may also cause compensatory movements in the other segments of the body, which further cause deviation from typical pattern.

Neuromuscular scoliosis is a secondary complication in children with SCI and it is strongly correlated with age at the time of injury with children at a higher risk of developing scoliosis than adults (Mulcahey et al., 2013; S. Parent et al., 2011). Neuromuscular scoliosis often includes pelvic obliquity and impaired trunk control (Driscoll \& Skinner, 2008). Current treatment strategies in SCI population 
allow for compensation for paralysis with braces and wheelchairs and with spinal fusion in children injured prior to twelve years of age (Sharma et al., 2013). These strategies neither restore the ability to sit upright nor resolve paralysis (Mehta, Betz, Mulcahey, McDonald, \& Vogel, 2004; S. Parent et al., 2011).

Lack of appropriate tools to evaluate trunk motor control following $\mathrm{SCl}$ restricts the ability to understand its development and therefore it's a challenge to design treatments and strategies to slow down or prevent the progress of longterm effects of SCl. International Standards for Neurological Classification of Spinal Cord Injury (ISNCSCI) scale is used in clinics to measure the effect and severity of SCl. However, trunk muscles are not included in the assessment (Allen et al., 2009; Chafetz et al., 2009; Stefan Parent et al., 2011). Various tests like the Trunk Impairment Scale (TIS), Gross Motor Function Classification System (GMFCS) have been used to measure trunk stability in children and adults. However, all these tests require that participants be able to sit or stand independently during data collection. Therefore, testing of trunk control in children with low functional level, i.e. those who have not achieved independent sitting, is limited (Saavedra \& Woollacott, 2015).

A new tool, the SATCo is used in clinics to assess trunk control on a segmental basis and used in typically TD children who have not developed independent sitting and in children with neuromotor disability (P. B. Butler et al., 2010; Curtis et al., 2015).

Therefore, assessment of trunk control during sitting may help to understand the impairment of trunk control in children with SCI. We hypothesized 
that children with $\mathrm{SCI}$ would produce lower activation of trunk muscles when compared to age matched TD children during SATCo testing 


\section{Methods}

Trunk control was assessed using SATCo test. Participants were tested in seated position with hip and knee both at 90 degrees of flexion with feet on ground and back unsupported. Simultaneously sEMG signals from various trunk muscles (RA, OB, PST, and PSL) were recorded. Refer to chapter III (page 49) for more details.

\section{Results}

Trunk motor control outcome (sEMG amplitude) was not significantly different between the TD children and children with SCI for all the different levels of SATCo test. Only for over lower ribs static control (OLRSC) level, the sEMG amplitude of trunk muscles was significantly lower in the preschool children with $\mathrm{SCl}$ compared to TD children in same age group (Figure VI.1).

When compared individual SATCo levels, paraspinal at lumbar level (PSL) muscle activation was significantly lower in school-age children with SCI compared to TD school-age children at ASC level (Figure VI.III). Paraspinal muscle at thoracic level (PST) muscle activation was significantly lower in preschool children with SCI compared to TD preschool children at inferior scapula static control level (ISSC) (Figure VI.IV).

Within SCI group, children with higher levels of $\mathrm{SCl}$ showed significantly lower trunk muscle activation at shoulder static control and (SSC) and axilla static control (ASC) levels compared to children with lower levels of $\mathrm{SCl}$ (Figure VI.VI). However, rests of the levels were not significantly different. When 
compared for muscles of lumbar region (lower trunk), children with lower levels of SCI showed significantly higher activation in muscles at SSC, ASC, BRSC and NSSC levels except at ISSC and OLRSC levels. 


\section{SATCo Levels}

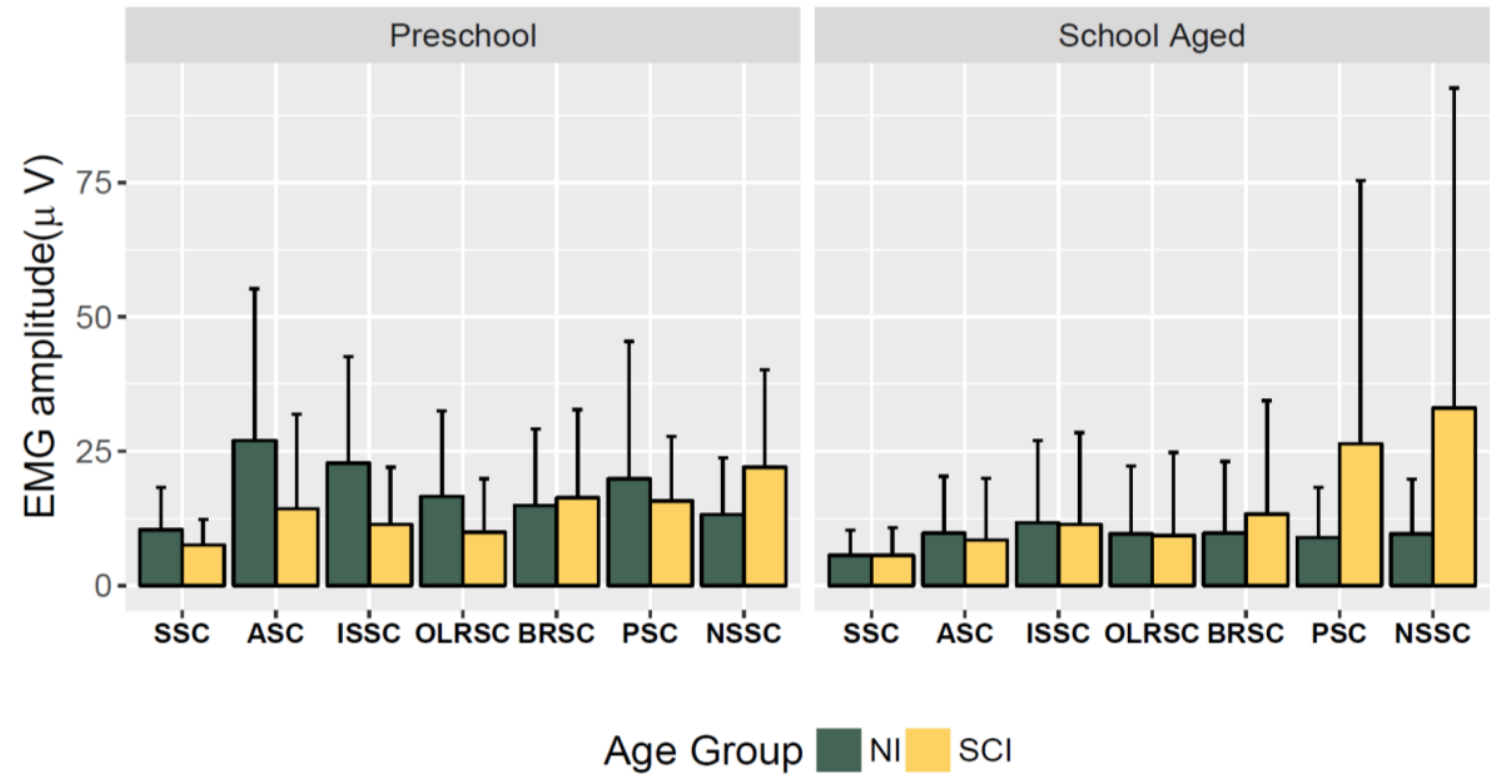

Figure VI:I: Trunk muscle activation during SATCo test in TD children with SCI

Shoulder static control (SSC), Axilla static control (ASC), Inferior scapula static control (ISSCI), Over lower ribs static control (OLRSC), Below ribs static control (BRSC), Pelvis static control (PSC) and No support static control (NSSC).

Activation Figure of the trunk muscles during the segmental assessment of trunk control testing. Overall activation of the trunk muscles, rectus abdominous, external oblique, thoracic paraspinal and lumbar paraspinal (microvolts) is plotted against different levels of trunk support during SATCo test. Electromyography amplitude $(\mu \mathrm{V})$ from various trunk muscles calculated as the root mean square (RMS) values is plotted between non-injured (NI) and children with $\mathrm{SCl}$ with their age-matched group i.e. preschool and school-age. For school-age groups, no significant differences were recorded for SSC ( $p=.53)$. ASC $(p=.22)$, ISSC $(p=.59)$ 
OLRSC $(p=.49), \operatorname{BRSC}(p=.09), \operatorname{PSC}(p=.06), \operatorname{NSSC}(p=.11)$ levels. The values are represented as a mean \pm standard deviation. In preschool group, at OLRSC level, children with $\mathrm{SCl}$ showed significantly lower $(p=.01)$ muscle activations compared to TD children in same age group. 
Shoulder Static Control

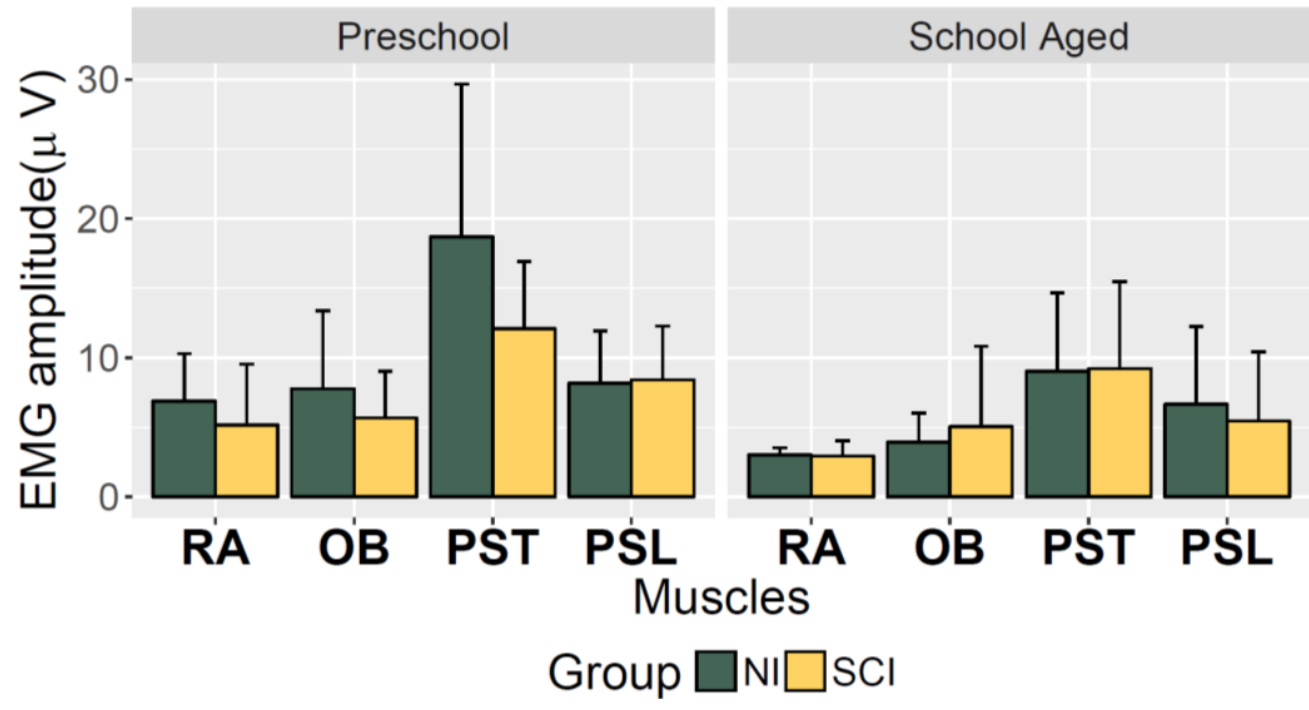

Figure VI:II: Trunk muscle activation during SSC in TD children with SCI

Rectus abdominus (RA), external oblique (OB), paraspinal at thoracic level (PST) and paraspinal at lumbar level (PSL).

Electromyography amplitude from various trunk muscles calculated as the root mean square (RMS) values in microvolts is plotted on the $y$-axis. RMS values of rectus abdominis (RA), external oblique (OB), paraspinal at thoracic (PST) and lumbar levels (PSL) calculated during testing at shoulder static control level of segmental assessment of trunk control test (SATCo) between non-injured (NI) and children with SCI with their age-matched group. For the preschool group, no significant differences were observed for RA ( $p=.31)$, PST ( $p=.21)$, OB $(p=.59)$, and PSL ( $p=.96)$ muscles activation. Similarly, for the school-Age group, no significant differences were recorded for RA ( $p=.56)$, PST ( $p=.90)$, OB $(p=.92)$ and PSL ( $p=.51)$ muscles activation at shoulder static level of support (SSC). The values are represented as a mean \pm standard deviation. 


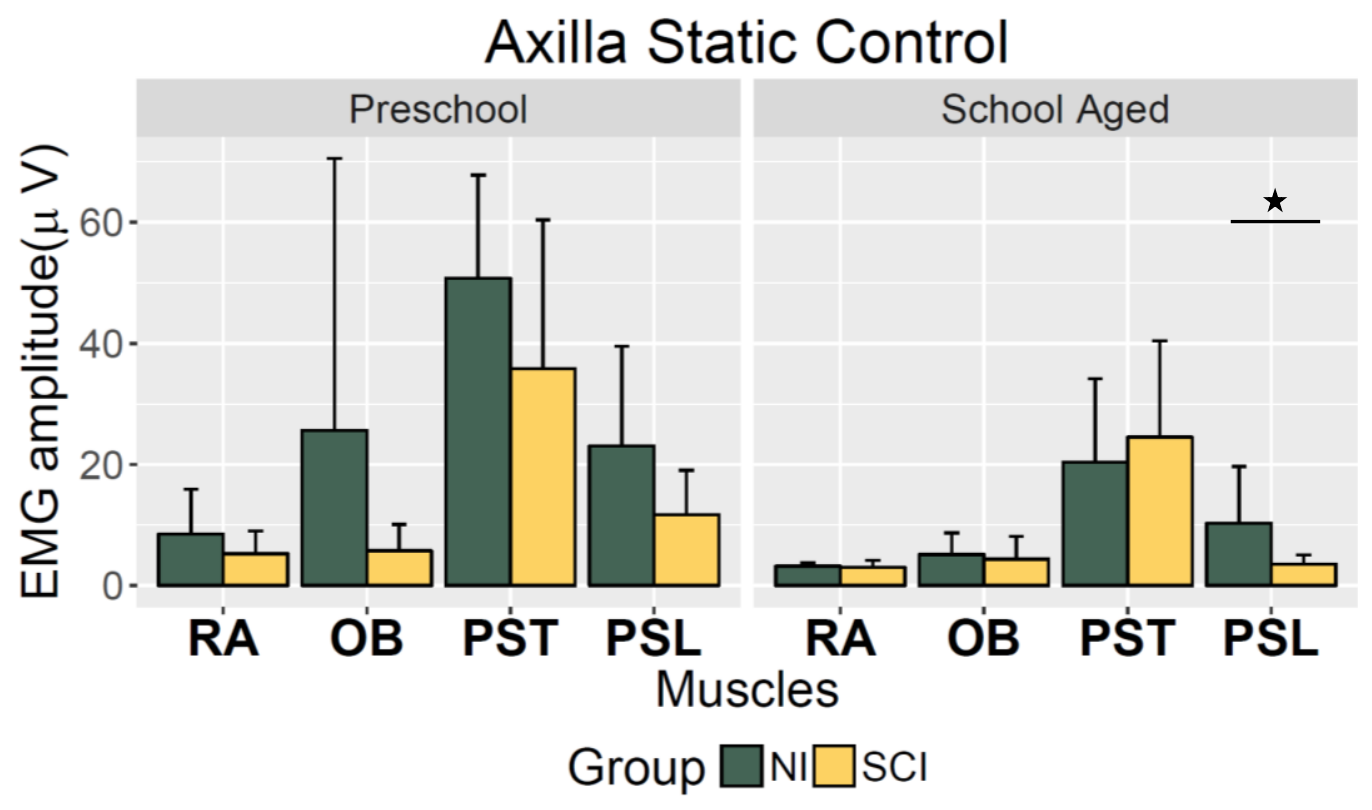

Figure VI:III: Trunk muscle activation during ASC in TD children with SCI

Rectus abdominus (RA), external oblique (OB), paraspinal at thoracic level (PST) and paraspinal at lumbar level (PSL).

Electromyography amplitude from rectus abdominis (RA), external oblique (OB), paraspinal at thoracic (PST) and lumbar levels (PSL) calculated during testing at Axilla static control level of segmental assessment of trunk control test (SATCo) between non-injured $(\mathrm{NI})$ and children with $\mathrm{SCl}$ with their age-matched group. For the preschool group, no significant differences were observed for $\operatorname{RA}(p=.28)$, PST ( $p=.15)$, OB $(p=.32)$, and PSL $(p=.28)$ muscles activation. Similarly, for the school-age group, no significant differences were recorded for RA ( $p=.52)$, PST $(p=.90)$ and $\mathrm{OB}(p=.47)$ muscles activation at axilla static control. However, PSL $(p=.04)$ muscles activation was significantly lower in school-age $\mathrm{SCl}$ group compared to TD children in same age group. The values are represented as a mean \pm standard deviation. 


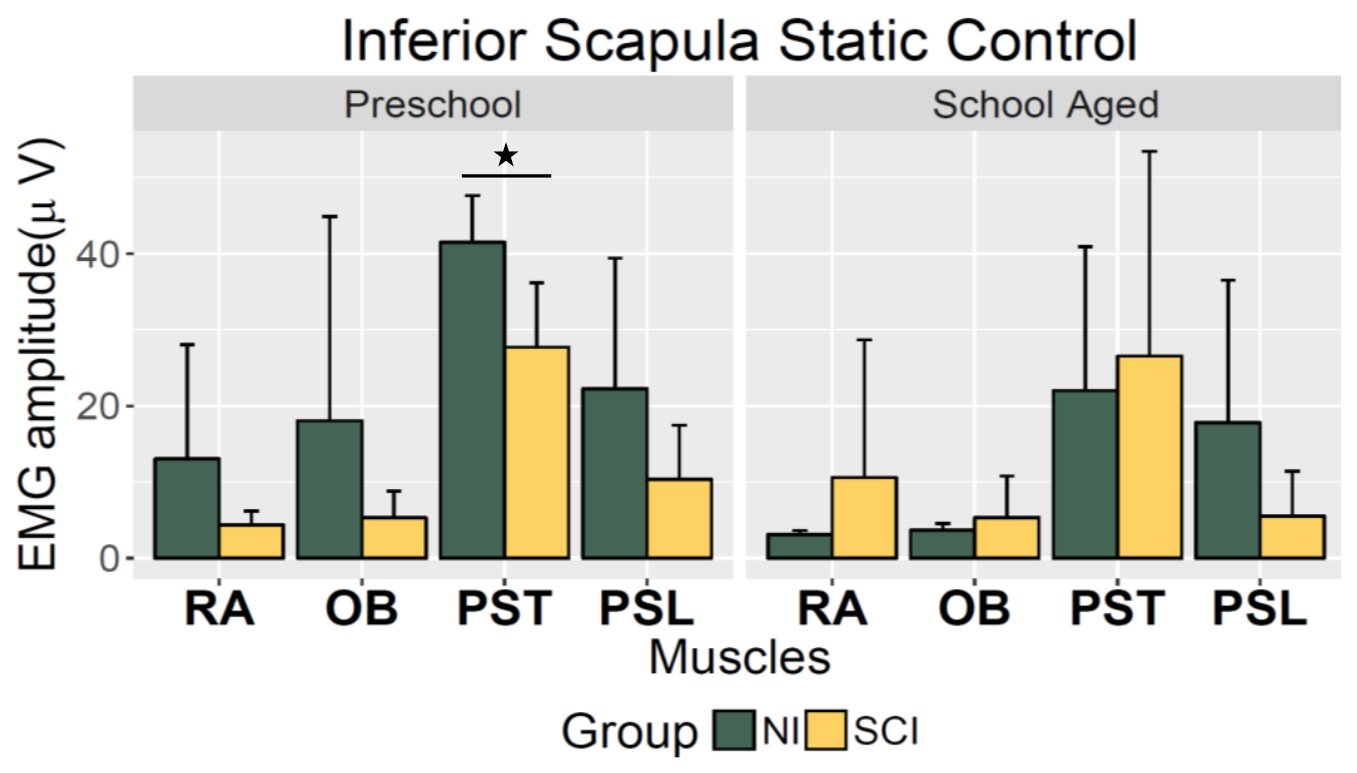

Figure VI:IV: Trunk muscle activation during ISSC in TD children with SCI

Rectus abdominus (RA), external oblique (OB), paraspinal at thoracic level (PST) and paraspinal at lumbar level (PSL).

Electromyography amplitude from rectus abdominis (RA), external oblique $(\mathrm{OB})$, paraspinal at thoracic (PST) and lumbar levels (PSL) calculated during testing at Inferior scapula static control level of segmental assessment of trunk control test (SATCo) between non-injured ( $\mathrm{NI}$ ) and children with $\mathrm{SCI}$ with their age-matched group. For the preschool group, no significant differences were observed for RA $(p=.09)$, OB $(p=.32)$, and PSL $(p=.29)$ muscles activation. However, PST $(p=.02)$ muscles activation was significantly lower in preschool $\mathrm{SCl}$ group. For the school-age group, no significant differences were recorded for RA ( $p=.26)$, PST $(p=.87), \mathrm{OB}(p=.75)$ and PSL $(p=.06)$ muscle activation at Inferior scapula static control. The values are represented as a mean \pm standard deviation. 
Over lower ribs static control (OLRSC)

In the preschool age group, no significant differences were observed between $\mathrm{NI}$ and children with SCI for RA ( $p=.15)$, PST ( $p=.13)$, OB ( $p=.63)$, and PSL $(p=.56)$ muscles activation. Similarly, for School-Age group, no significant differences were observed for RA $(p=.19)$, PST $(p=.90)$, OB $(p=.45)$ and PSL $(p=.12)$ muscles activation at over lower ribs static level of support.

Below ribs static control (BRSC)

For the preschool group, no significant differences were recorded between NI and children with SCI for RA ( $p=.51)$, PST $(p=.16)$, OB ( $p=.28)$, and PSL $(p=.98)$ muscle activation. Similarly, for School-age group, there were no significant differences between the two groups for activation of RA ( $p=.32)$, PST ( $p=.20)$, OB $(p=.85)$ and PSL $(p=.29)$ muscles at below ribs level. 


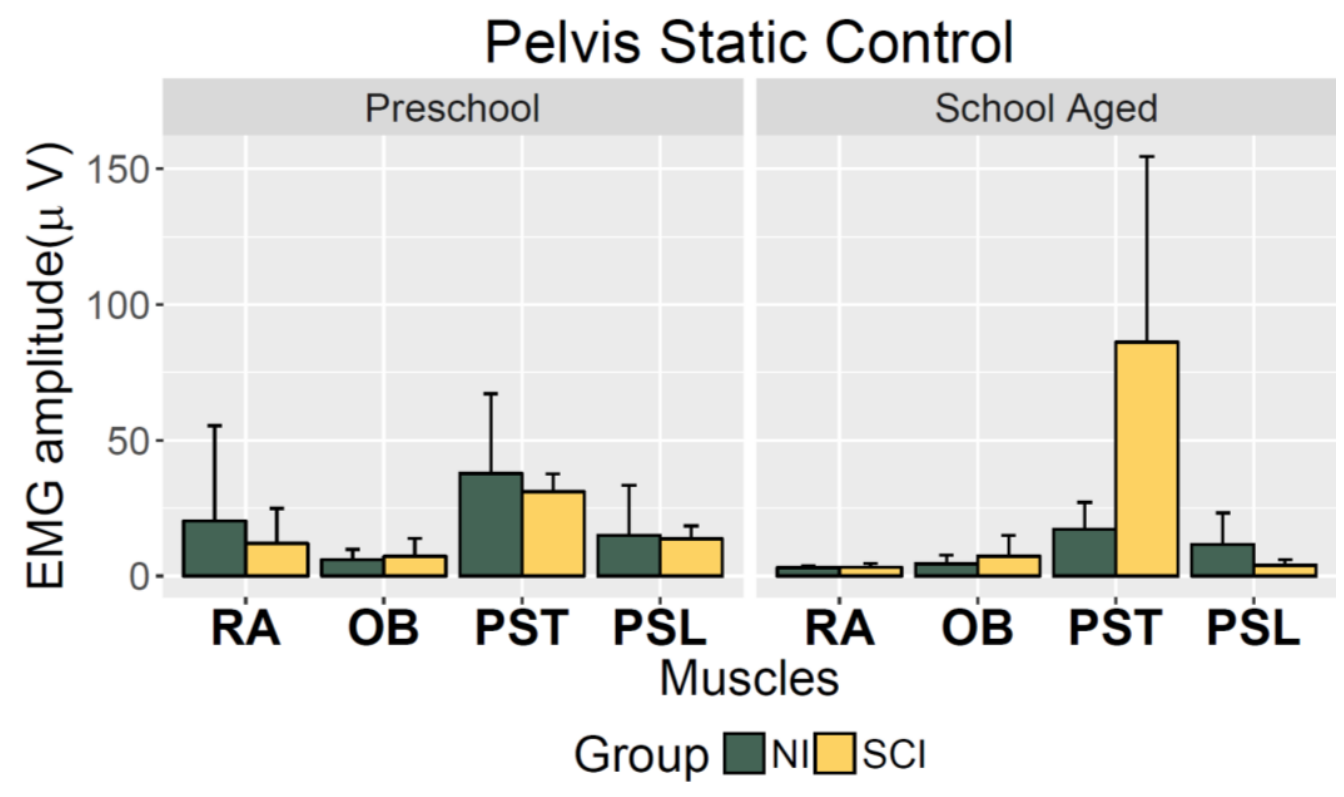

Figure VI:V: Trunk muscle activation during PSC in TD children with SCI

Rectus abdominus (RA), external oblique (OB), paraspinal at thoracic level (PST) and paraspinal at lumbar level (PSL).

Electromyography amplitude from rectus abdominis $(R A)$, external oblique $(O B)$, paraspinal at thoracic (PST) and lumbar levels (PSL) calculated during testing at pelvis static control level of segmental assessment of trunk control test (SATCo) between non-injured (NI) or typically developing (TD) and children with $\mathrm{SCl}$ with age-matched group. For the preschool group, no significant differences were observed for RA ( $p=.91)$, OB ( $p=.32)$, PSL $(p=.61)$ and PST $(p=.98)$ muscles activation between TD and SCI. Similarly, for the school-age group, no significant differences were recorded between $\mathrm{NI}$ and $\mathrm{SCI}$ groups for RA ( $p=.90)$, PST $(p=.06), \mathrm{OB}(p=.48)$ and PSL $(p=.13)$ muscle activation at pelvis static control level. The values are represented as a mean \pm standard deviation. 


\section{No support static control}

For the preschool group, no significant differences were observed for $\mathrm{RA}(p=.69)$, PST $(p=.10)$, OB $(p=.13)$, and PSL $(p=.79)$ muscles activation. Similarly, for School-Age group, no significant differences were recorded for RA ( $p=.57)$, OB $(p=.56)$ and PSL $(p=.27)$ muscle activation at SSC level of support. However, only PST $(p=.01)$, muscle activation was significantly higher in school-age children with $\mathrm{SCl}$ compared to TD children in same age group. 


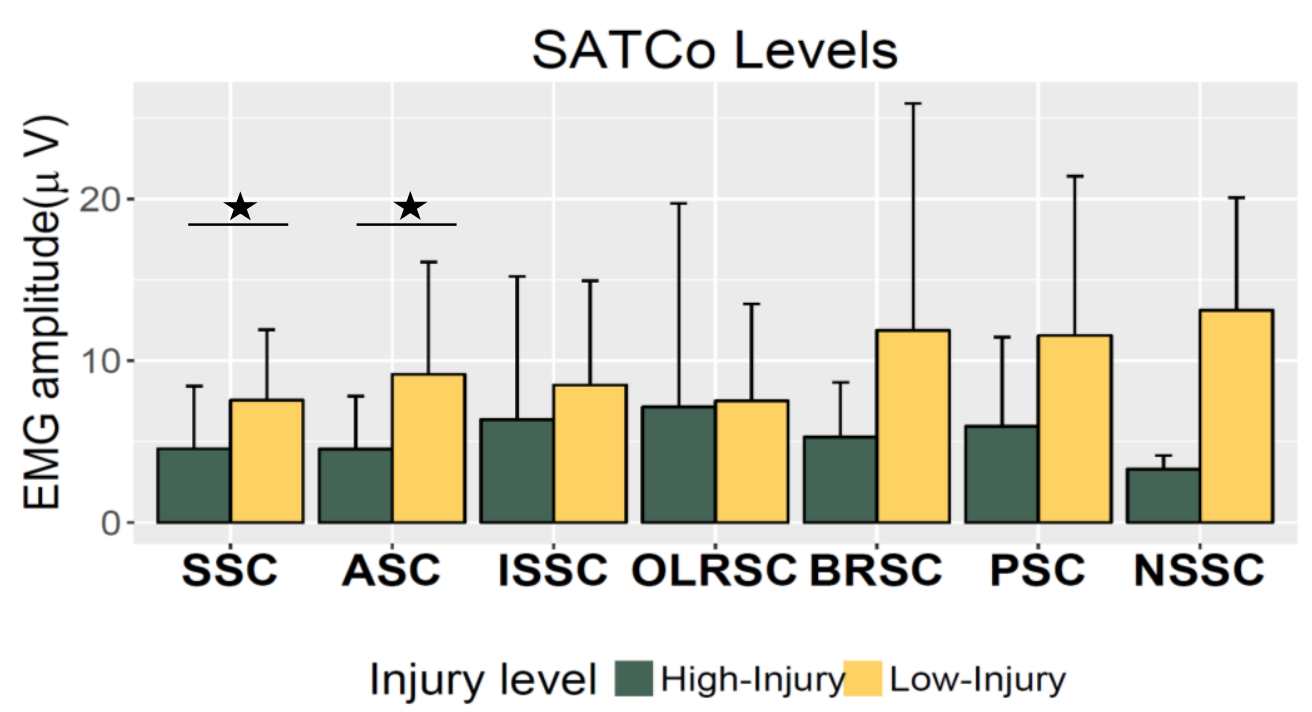

Figure VI:VI: Trunk muscle activation during SATCo test in children with SCI

Electromyography amplitude from combined trunk muscles, i.e. rectus abdominous (RA), external oblique (OB) thoracic (PST) and lumbar paraspinal (PSL) are plotted for each SATCo level between children with low and high levels of SCl. There were significant differences between the children with higher levels of $\mathrm{SCl}$ and those with lower levels of $\mathrm{SCl}$ for shoulder static control (SSC) $(p=.01)$ and axilla static control (ASC) $(p=.03)$ level of support, i.e. children with lower levels of $\mathrm{SCl}$ produced higher activation of trunk muscles than children with higher levels of SCl. However, no significant differences were reported for rest of the SATCo levels with inferior scapula static control (ISSC) $(p=.11)$, Over lower ribs static control (OLRSC) $(p=.06)$, below ribs static control (BRSC) $(p=.07)$, pelvis static control (PSC) ( $p=.31)$, and no support static control (NSSC) $(p=.34)$ between children with higher and lower levels of SCl. The values are represented as a mean \pm standard deviation. 


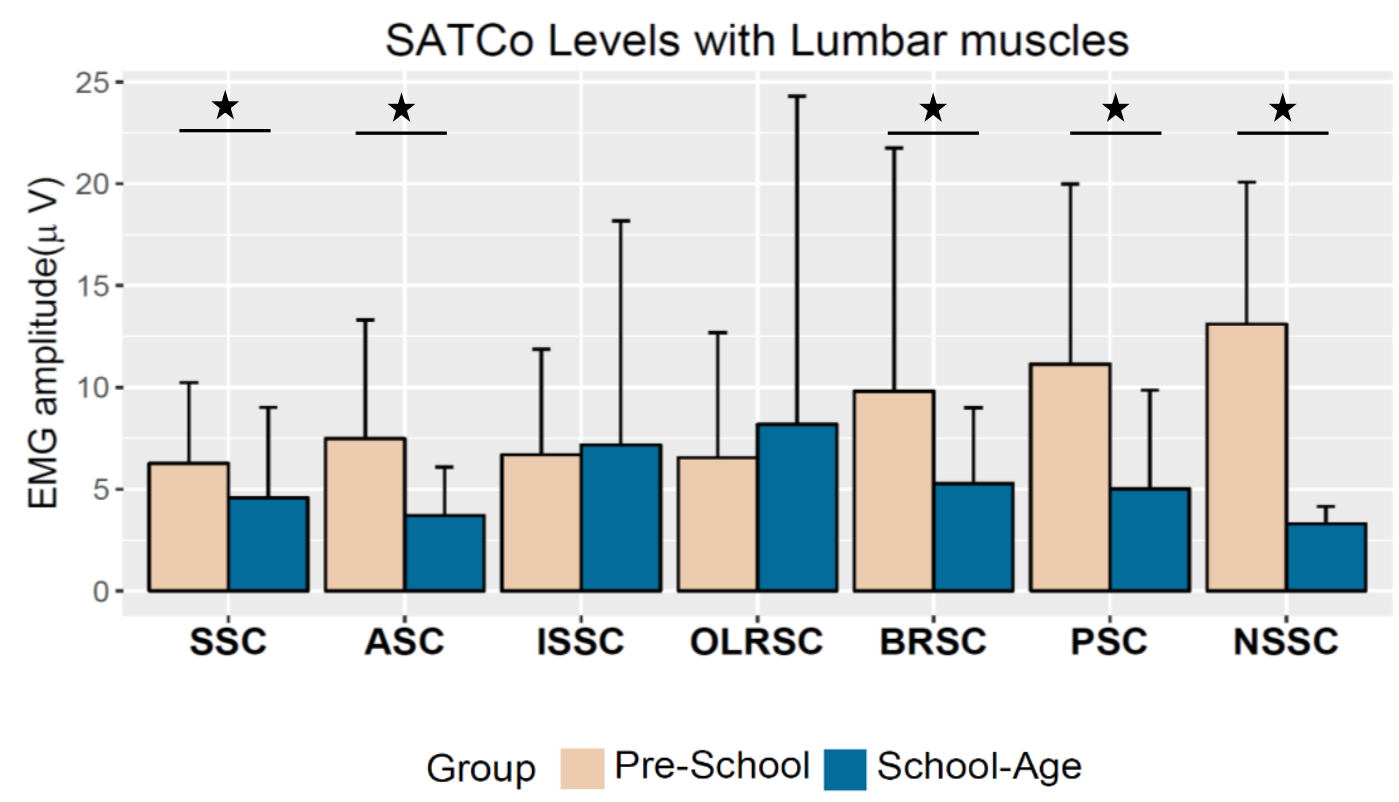

Figure VI:VII: Trunk muscle activation during SATCo in children with SCI

Shoulder static control (SSC), Axilla static control (ASC), Inferior scapula static control (ISSCI), Over lower ribs static control (OLRSC), Below ribs static control (BRSC), Pelvis static control (PSC) and No support static control (NSSC).

Electromyography amplitude of only lumbar segment muscles (RA, OB, and PSL) are plotted for each SATCo level for children with SCI between preschool and school age. Preschool children with $\mathrm{SCl}$ had higher activation in lumbar muscles for all levels of SATCo support compared to school age children with SCI. Trunk muscles activation was significantly higher at shoulder (SSC) $(p=.04)$ axilla (ASC) ( $p=.005)$, below ribs (BRSC) $(p=.07)$, pelvis (PSC) $(p=.01)$ and no support static control (NSSC) ( $p=.005)$. However, muscle activation was not significantly different between preschool and school-age SCl children for inferior scapula (ISSC) $(p=.32)$ and over lower ribs static control (OLRSC) $(p=.49)$. The values are represented as a mean \pm standard deviation 


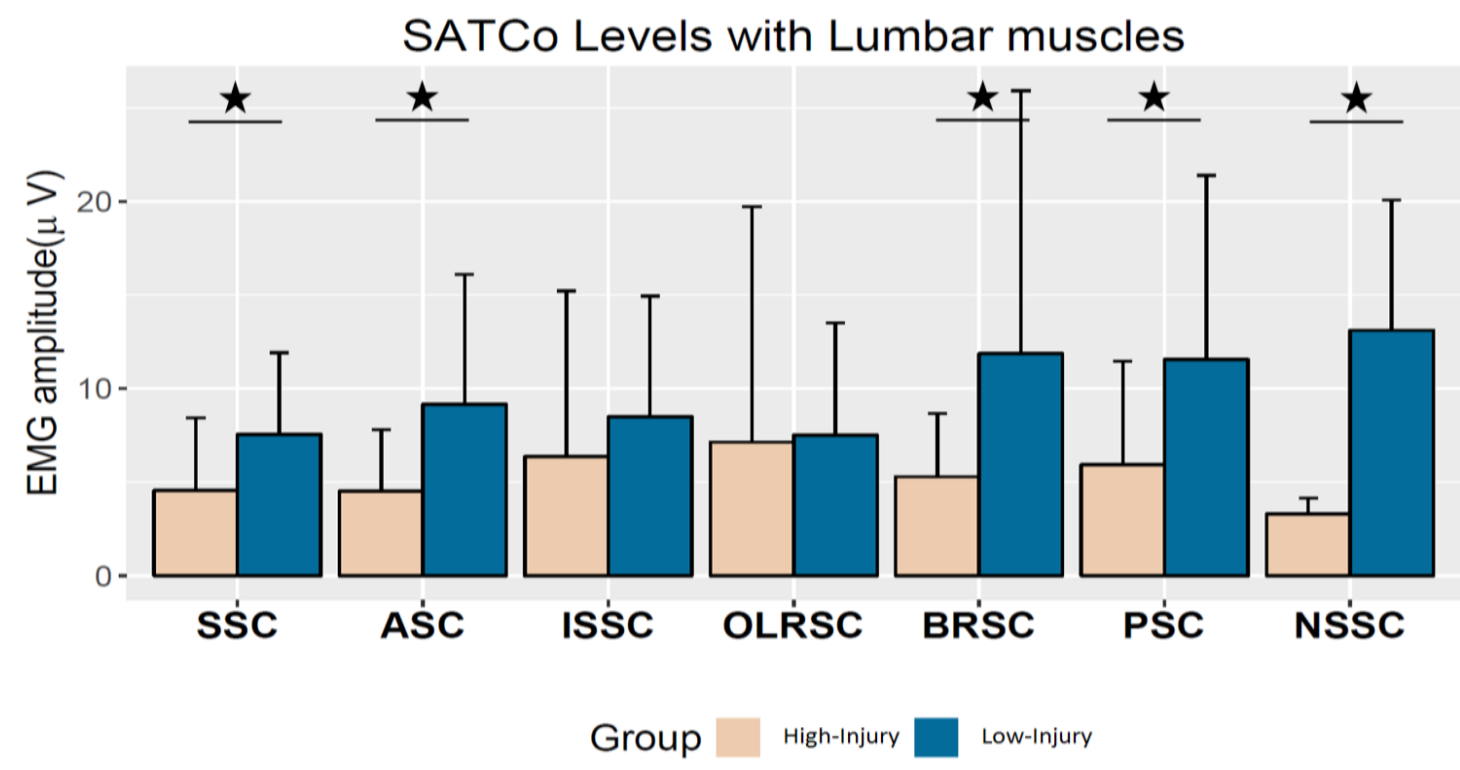

Figure VI:VIII: Trunk muscle activation during SATCo in children with SCI

Electromyography amplitude of only lumbar segment muscles (RA, OB, and PSL) are plotted for each SATCo level between children with lower and higher levels of SCl. Children with lower levels of SCl had significantly higher activation of lumbar trunk muscles at shoulder (SSC) $(p=.01)$, axilla (ASC) $(p=.006)$, below ribs (BRSC) ( $p=.03)$ pelvis (PSC) $(p=.05)$ and no support static control (NSSC) $(p=.005)$ levels of SATCo support than compared to children with higher levels of SCI. However, muscle activation was not significantly different between the two groups for inferior scapula (ISSC) $(p=.11)$ and over lower ribs static control (OLRSC) $(p=.31)$. The values are represented as a mean \pm standard deviation. 


\section{Discussion}

For this part of the study, we examined sEMG amplitude signals from different trunk muscles during SATCo test between TD children (NI) and children with SCI to their age-matched counterpart i.e. preschool and school-age. We found no significant differences between the two groups for sEMG amplitude for all SATCo levels, except for over lower ribs static control level, where children in $\mathrm{SCl}$ produced lower activation of trunk muscles compared to TD children in preschool age group (Figure VI.I).

We also looked at individual muscle activation for each level of SATCo support, but we found no significant differences between the two groups. Even though we consistently observed lower activation of trunk muscles in preschool children with $\mathrm{SCl}$, however, differences were not statistically significant. It is important to note that not every child with $\mathrm{SCl}$ was able to complete the test for all the SATCo levels so, comparison of muscle activation (sEMG) was done only if they completed that SATCo level. In other words, the sEMG data from children with SCI for a specific level of SATCo support was analyzed and compared to TD children only if they were able to maintain trunk control for that level of SATCo test. This indicates that SATCo test is reliable for measuring trunk control and is sensitive to level of lesion as it corresponds to the spinal segment being tested.

Interestingly, when we divided SCI subject into two groups; lower level and higher levels of $\mathrm{SCl}$, we found that children with lower levels of $\mathrm{SCl}$ had higher activation of trunk muscles for all the SATCo levels, but statistically significant for only first two levels (Figure V.6). However, when only lumbar 
segment muscles (RA, OB, and PSL) were analyzed, 6 out 7 SATCo levels were significantly different between children with higher and lower levels of SCI (Figure VI.VIII). Children with higher levels of SCI had lower muscle activation compared to children to children with lower levels of $\mathrm{SCl}$, who produced higher activation in trunk muscles. These results indicate that levels of $\mathrm{SCl}$ can have an impact on trunk motor control outcomes as measured by sEMG during SATCo test.

We also found that age had a significant effect on trunk motor control outcomes as measured by SATCo test. Preschool children with SCI had increased activation in lumbar segment muscles (RA, OB, and PSL) compared to school age children with $\mathrm{SCl}$, who showed decreased muscle activation at the same region (Figure VI.VII). These results are consistent as observed in preschool and school age TD children, where younger (preschool) children had higher activation in trunk muscles than older children, indicative of variability in postural muscles response associated with age in both children with and without $\mathrm{SCl}$. This also raises the question of when these postural muscle synergies emerge in children with $\mathrm{SCl}$ and if rehabilitation and training might have a role to play. This information will also help and guide therapist in evaluating and treating postural impairments in seated position.

Interestingly, children with $\mathrm{SCl}$ showed a consistent higher activation in thoracic paraspinal muscle for all SATCo levels. A possible explanation for this could be that children with $\mathrm{SCI}$ were compensating for the loss of other postural muscles needed to maintain static trunk control. Postural tone in trunk muscles is considered as the major mechanism in supporting the body against force of 
gravity therefore, baseline tonic activation of paraspinal muscles is necessary to maintain seated upright position. In a study investigating muscle activation before a seated researching task in adults reported a significant baseline tonic muscle activation in lumbar, thoracic and cervical paraspinal muscles during steady seated state. This indicates the importance of tonic activation of paraspinal muscles in maintaining steady seated trunk control.

The present findings of this study strengthen the concept of altered/impaired postural control in children with SCI. 


\section{$\&+\$ 37$ ( $59,, \quad$ RESPIRATORY MOTOR CONTROL IN CHILDREN WITH $63,1 \$$ / CORD ,1-85< \\ ,ntroduction}

The imbalance between respiratory muscle load and capacity can lead to respiratory insufficiency. Children with neuromuscular disorders develop respiratory insufficiency due to paralysis and or weakness of muscles associated with respiration. Higher cervical and upper thoracic injury disrupts the function of diaphragm, intercostal, abdominal and accessory muscles of respiration (Finkel, Weiner, Mayer, McDonough, \& Panitch, 2014; Nicot et al., 2006; Schilero et al., 2009). Paralysis or spasticity following SCI can cause a reduction in lung volumes and weak or inability to a cough. This further results in accumulation of bronchial secretions, mucus retention, atelectasis, pulmonary infections which results in significant morbidity and mortality (Schilero et al., 2009; Terson de Paleville \& Lorenz, 2015; Warren et al., 2014).

$\mathrm{SCl}$ in children can have severe consequences compared to $\mathrm{SCl}$ in adults because children are still undergoing motor development. The rib cage in children lacks the mechanical efficiency, as is it more circular than elliptical like in adults. Due to the position of ribs at a right angle to the vertebral column, children have limited ability to expand their ribs during inspiration with less tidal volume 
(De Troyer, Estenne, \& Vincken, 1986; De Troyer \& Heilporn, 1980). Children also have inherent higher compliance of chest wall relative to lung compliance, which predisposes to have low functional residual volume.

During the process of growth and development, there is a progressive increase in the size of respiratory muscles, changes in fiber type composition, fiber size and oxidative capacity of the diaphragm muscle. Also, children have less fatigue resistance Type-I fibers, but a higher proportion of Type-llc fatigue susceptible fibers, therefore, in children diaphragm muscle is prone to early fatigue than in adults (Leung et al., 2012). Pneumonia is the leading respiratory complications in children and adults with SCl (Claxton et al., 1998; Estenne \& Gorini, 1992; Fishburn et al., 1990; Jackson \& Groomes, 1994; Schilero et al., 2009).

Neuromuscular scoliosis is another complication that results in children after SCl. There is almost $100 \%$ chance that a child will develop scoliosis if they get injured before the age of 10 years. Neuromuscular scoliosis further decreases the mechanical efficiency of the chest wall thereby reducing lung functions (Mulcahey et al., 2013; Zaba, 2002, 2003a, 2003b). As these children continue to develop and attain maturity, there occur dynamic changes in their musculo-skeletal system simultaneously affecting lung volumes and static mouth pressures. Therefore, children with $\mathrm{SCl}$ are particularly at high risk for developing respiratory complications and it becomes crucial that these patients are evaluated for respiratory function as early and as frequently as possible. 
Pulmonary function testing including, $\mathrm{PE}_{\max }$ and $\mathrm{PI} \max$ are important tools that are used in the clinic to diagnose, assess and manage respiratory diseases, both in adults and children. However, these assessments do not provide information about the underlying neural drive to the respiratory motor system. Evaluation of respiratory functions in conjunction with recording sEMG signals from respiratory muscles will enable us to understand the involvement of the different respiratory muscles and severity of muscle weakness. The aim of this part of the study is to evaluate respiratory motor functions in children with $\mathrm{SCl}$ and compare their results to TD children. We hypothesized that children with SCI would produce lower lung volumes and airway pressures associated with lower activation of respiratory muscles when compared to age matched TD children.

\section{Methods}

A total of 14 TD children and 12 children with SCI completed the respiratory motor control testing with 5 and 6 children in preschool TD and SCI group, respectively. In the school-age group, we had 9 children in TD and 6 in $\mathrm{SCI}$ group. Demographics and respiratory measurements of children in TD and $\mathrm{SCI}$ groups are listed in table VI.I\&II and VII.I\&II, respectively. First, children in both groups were asked to perform standard pulmonary function testing (FVC, $\left.F E V_{1}\right)$. After spirometry, subjects were tested for maximum expiratory ( $\left.P E_{\max }\right)$ and inspiratory airway pressure $\left(\mathrm{PI} \mathrm{I}_{\max }\right)$ maneuvers while we simultaneously recorded surface electromyography from upper trapezius, pectoralis major, external intercostal, rectus abdominous, external oblique, paraspinal at thoracic and lumbar region. 


\section{Results}

Pulmonary function outcomes were significantly decreased in both, the preschool and school age children with SCI when compared to their agematched $\mathrm{NI}$ children: FVC, FEV 1 , PEmax and $\mathrm{PI}_{\max }$ (except FVC, $\mathrm{FEV}_{1} \mathrm{PI}_{\max }$ in preschool children with SCI) (Figure VII.I-VIII). SCI children in the school age group produced significantly lower values of $\mathrm{FVC}$ and $\mathrm{FEV}_{1}$ when compared to $\mathrm{NI}$ children in the same age group. However, no significant differences were found between $\mathrm{NI}$ and $\mathrm{SCl}$ children in the preschool group for FVC values, but $\mathrm{FEV}_{1}$ values were significantly decreased in preschool children with $\mathrm{SCl}$. Children with $\mathrm{SCl}$ (both age groups) showed absent or decreased RA \& OB muscles activation during expiratory airway pressure maneuver $\left(P E_{\max }\right)$ when compared to age-matched NI children (Figure VII.VII.B).

Children with $\mathrm{SCl}$ in the school-age group also showed significantly higher activation of UT \& PEC muscles (above the spinal lesion) during PEmax when compared to $\mathrm{NI}$ children in same age group. No significant differences in muscle activation were recorded between children with $\mathrm{SCI}$ and $\mathrm{NI}$ children (except higher activation in UT muscle in school age group) during PImax maneuver (Figure VII.VIIIB). 
Table VII-I: Preschool-Age- Children with Spinal Cord Injury for Respiratory Motor Control Assessment

\begin{tabular}{|c|c|c|c|c|c|c|c|c|c|c|c|c|}
\hline $\begin{array}{r}\text { Subject } \\
\text { (ID) }\end{array}$ & $\begin{array}{c}\text { Age } \\
\text { (years) }\end{array}$ & Gender & $\begin{array}{l}\text { Height } \\
\text { (cm) }\end{array}$ & $\begin{array}{c}\text { Weight } \\
\text { (Kgs) }\end{array}$ & $\begin{array}{l}\text { PEmax } \\
\text { (cmH20) }\end{array}$ & $\begin{array}{l}\text { PImax } \\
\text { (cmH20) }\end{array}$ & $\begin{array}{l}\text { FVC } \\
\text { (Liters) }\end{array}$ & $\begin{array}{l}\text { FEV }_{1} \\
\text { (Liters) }\end{array}$ & FVC\%P & FEV1\%P & $\begin{array}{l}\text { Injury } \\
\text { Level }\end{array}$ & $\begin{array}{c}\text { Time since } \\
\text { injury } \\
\text { (months) }\end{array}$ \\
\hline P3 & 4 & $M$ & 97 & 14 & 30 & -28 & 0.59 & 0.59 & 103 & 109 & $\mathrm{~T} 2$ & 50 \\
\hline P7 & 4 & $\mathrm{~F}$ & 114 & 20 & 13 & -19 & 0.59 & 0.58 & 50 & 54 & C5 & 30 \\
\hline P8 & 4 & $\mathrm{~F}$ & 112 & 28 & 25 & -29 & 0.75 & 0.68 & 78 & 66 & $\mathrm{~T} 2$ & 11 \\
\hline P9 & 5 & $\mathrm{~F}$ & 114 & 23 & 30 & -46 & 1 & 1 & 103 & 105 & C5 & 58 \\
\hline P14 & 5 & $M$ & 101 & 15 & 31 & -41 & 0.8 & 0.71 & 103 & 98 & $\mathrm{~T} 2$ & 28 \\
\hline P16 & 5 & $M$ & 109 & 19 & 58 & -54 & 1.5 & 1.2 & 128 & 122 & $\mathrm{~T} 12$ & 17 \\
\hline Mean \& SD & $4 \pm 0.5$ & $3 F, 3 M$ & $107 \pm 6$ & $19 \pm 4$ & $31 \pm 11$ & $-36 \pm 13$ & $0.8 \pm 0.3$ & $0.7 \pm 0.2$ & $94 \pm 24$ & $92 \pm 24$ & NA & $32 \pm 16$ \\
\hline
\end{tabular}


Table VII-II: School-Age- Children with Spinal Cord Injury for Respiratory Motor Control Assessment

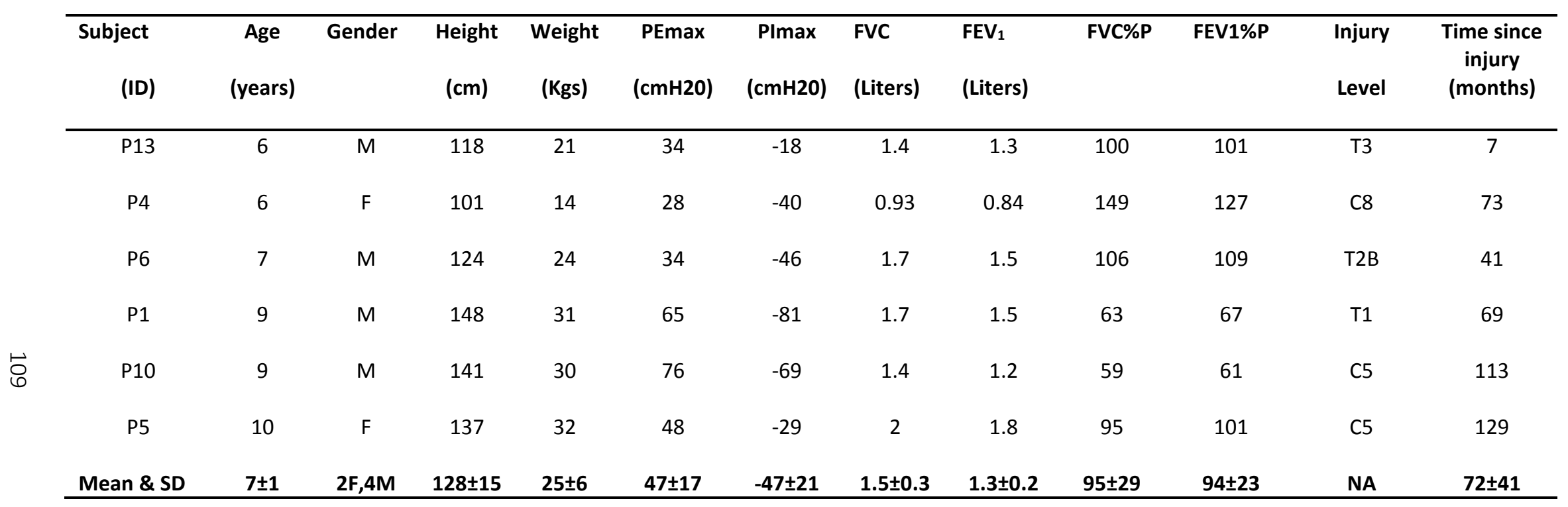




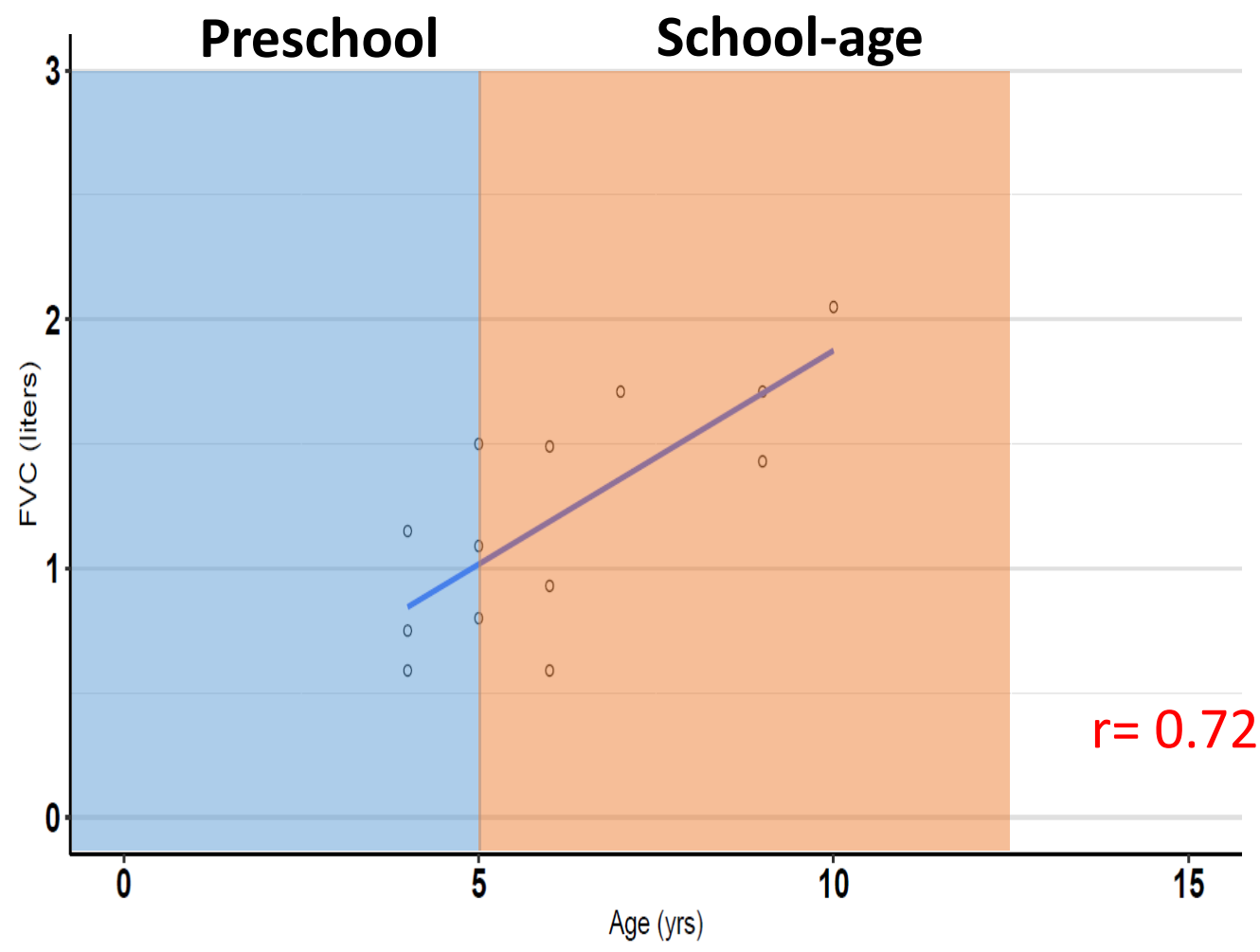

Figure VII:I: FVC in children with SCI

Correlation between Forced Expiratory Volume in one second (liters) and age (years) in children with SCI. The correlation between age and FVC was weaker in children with $\mathrm{SCl}(r=.72)$. 


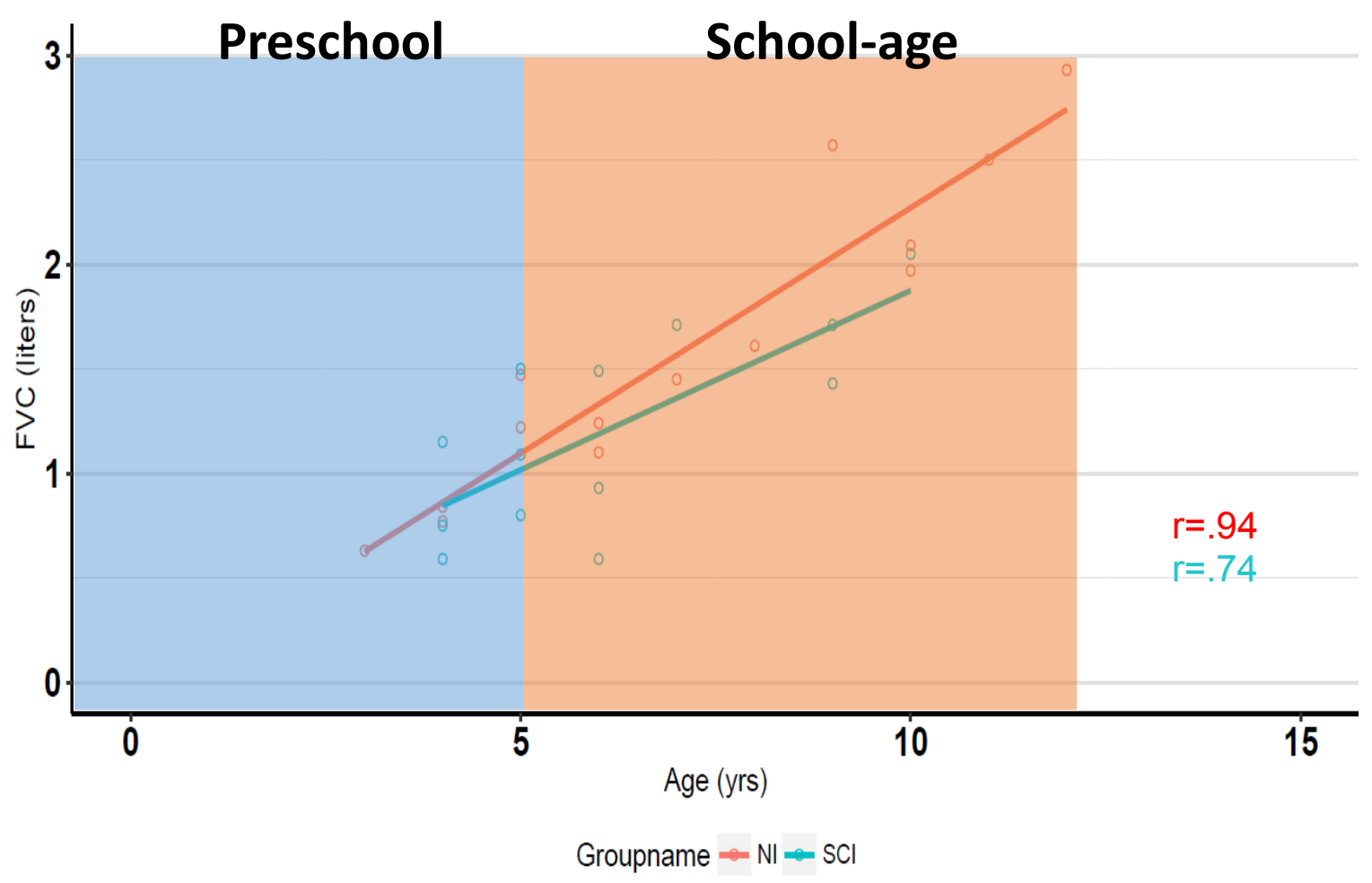

Figure VII:II: FVC in TD and children with SCI

Correlation between Forced Vital There was a linear relationship between FVC and age for children in TD group ( $r=.92)$. However, this correlation was weaker in children with $\mathrm{SCl}$ group $(r=.74)$. 


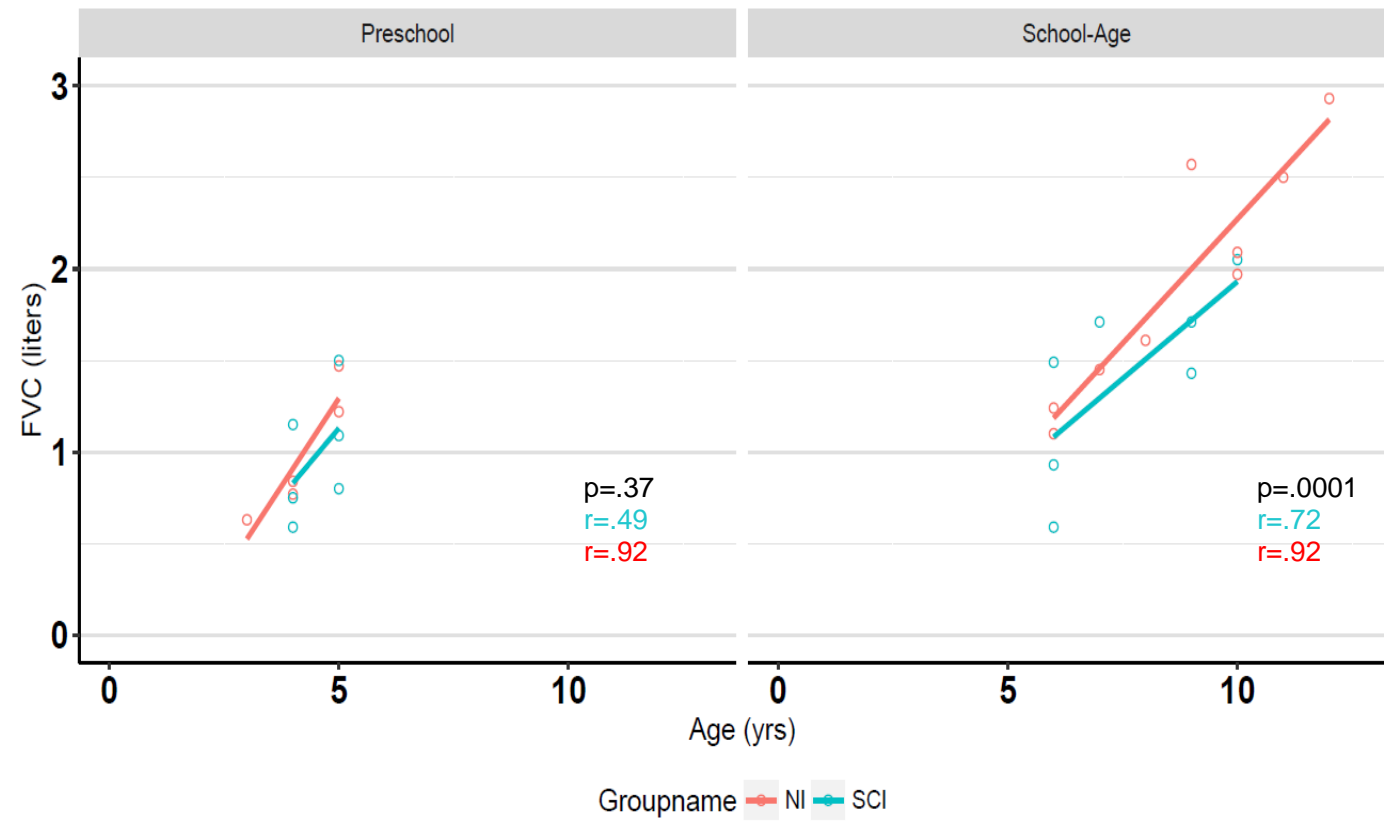

Figure VII:III: FVC in TD and in children with SCI

Correlation between Forced Vital Capacity (liters) and age (years) between noninjured and children with $\mathrm{SCl}$ age matched. A significant difference was observed between school-age $\mathrm{NI}$ and school age SCI groups $(p=.0001)$ i.e. $\mathrm{NI}$ children in school age group produced an increased volume of air (FVC) than children with $\mathrm{SCl}$ in same age group. No significant differences were found between $\mathrm{NI}$ and $\mathrm{SCI}$ children in the preschool group ( $p=0.37)$. There was a linear relationship between FVC and age for children in both the preschool ( $r=.92)$ and school age children ( $r=.92)$. However, this correlation was weaker in preschool children with SCI ( $r=.49)$, whereas school age children with SCl showed a moderately strong relationship ( $r=.72)$ between age and FVC. 


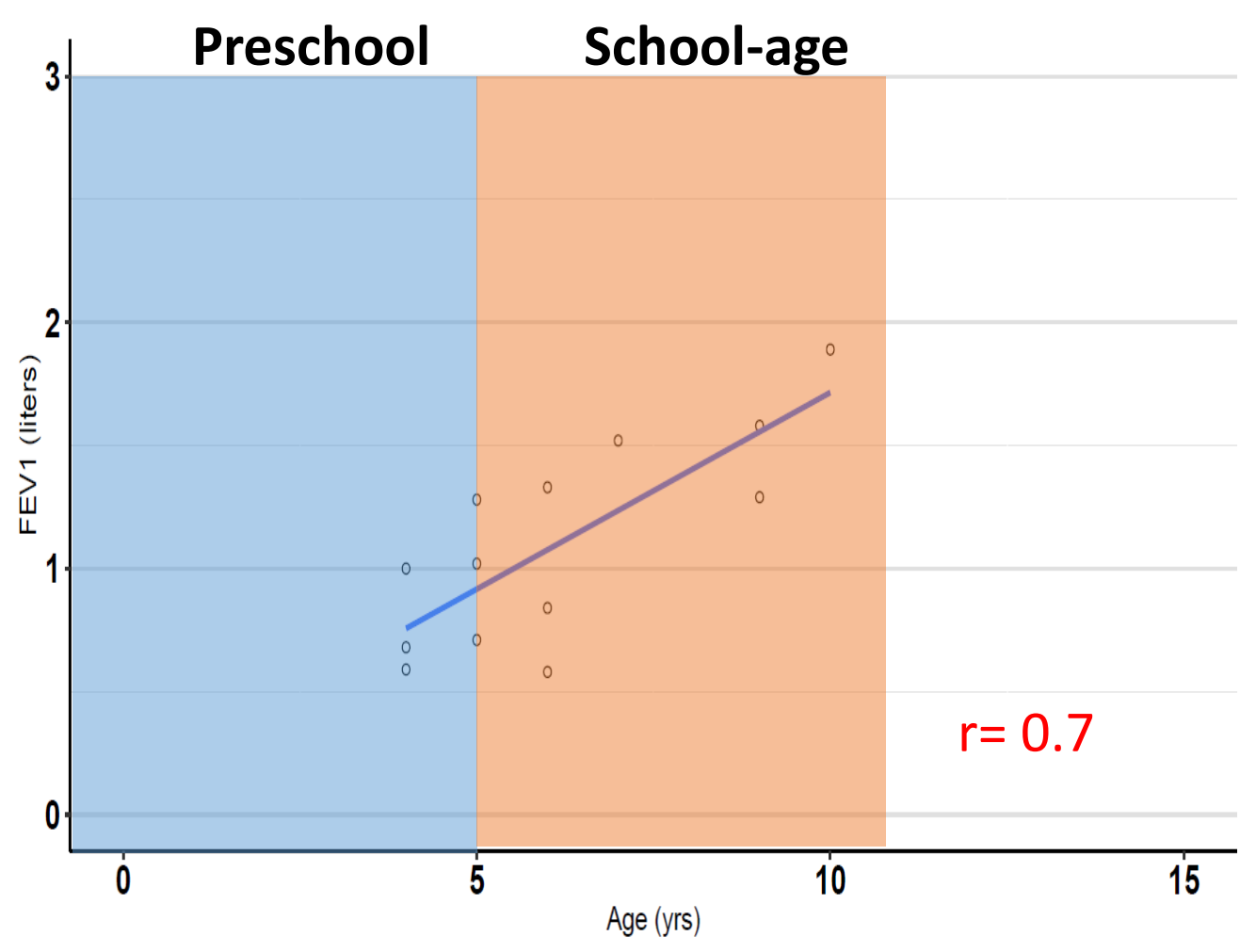

Figure VII:IV: $\mathrm{FEV}_{1}$ in children with $\mathrm{SCI}$

Correlation between Forced Expiratory Volume in one second (liters) and age (years) age (years) in children with SCI. The correlation was weaker in children with $\mathrm{SCl}(r=.70)$. 


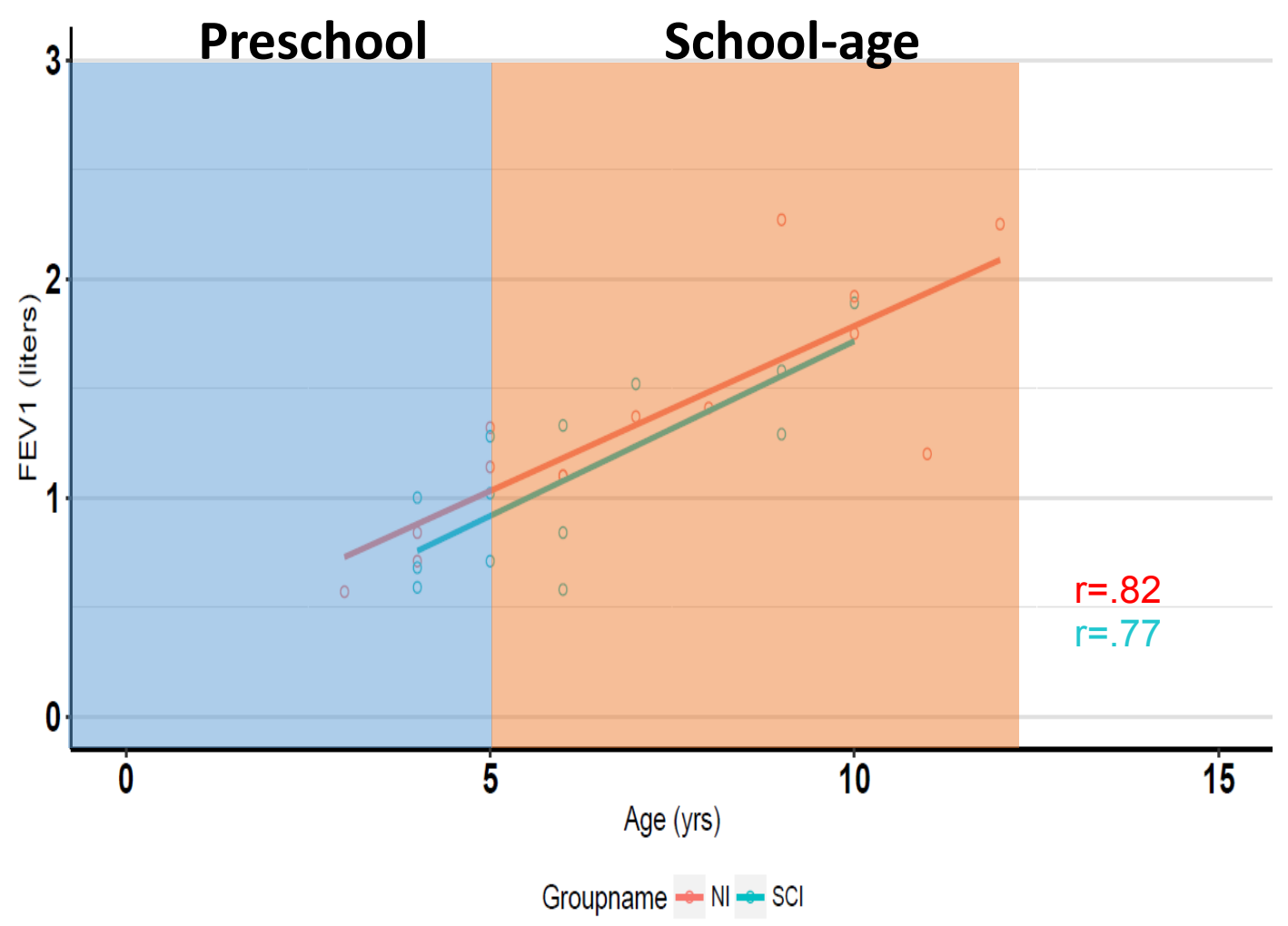

Figure VII:V: $\mathrm{FEV}_{1}$ in children with $\mathrm{SCI}$

Correlation between Forced Expiratory Volume in one second (liters) and age (years) between TD and children with $\mathrm{SCl}$ as two groups. There was a linear relationship between $\mathrm{FEV}_{1}$ and age for children in TD group ( $\left.r=.82\right)$ However, this correlation was weaker in children with SCI group ( $r=.77)$. 


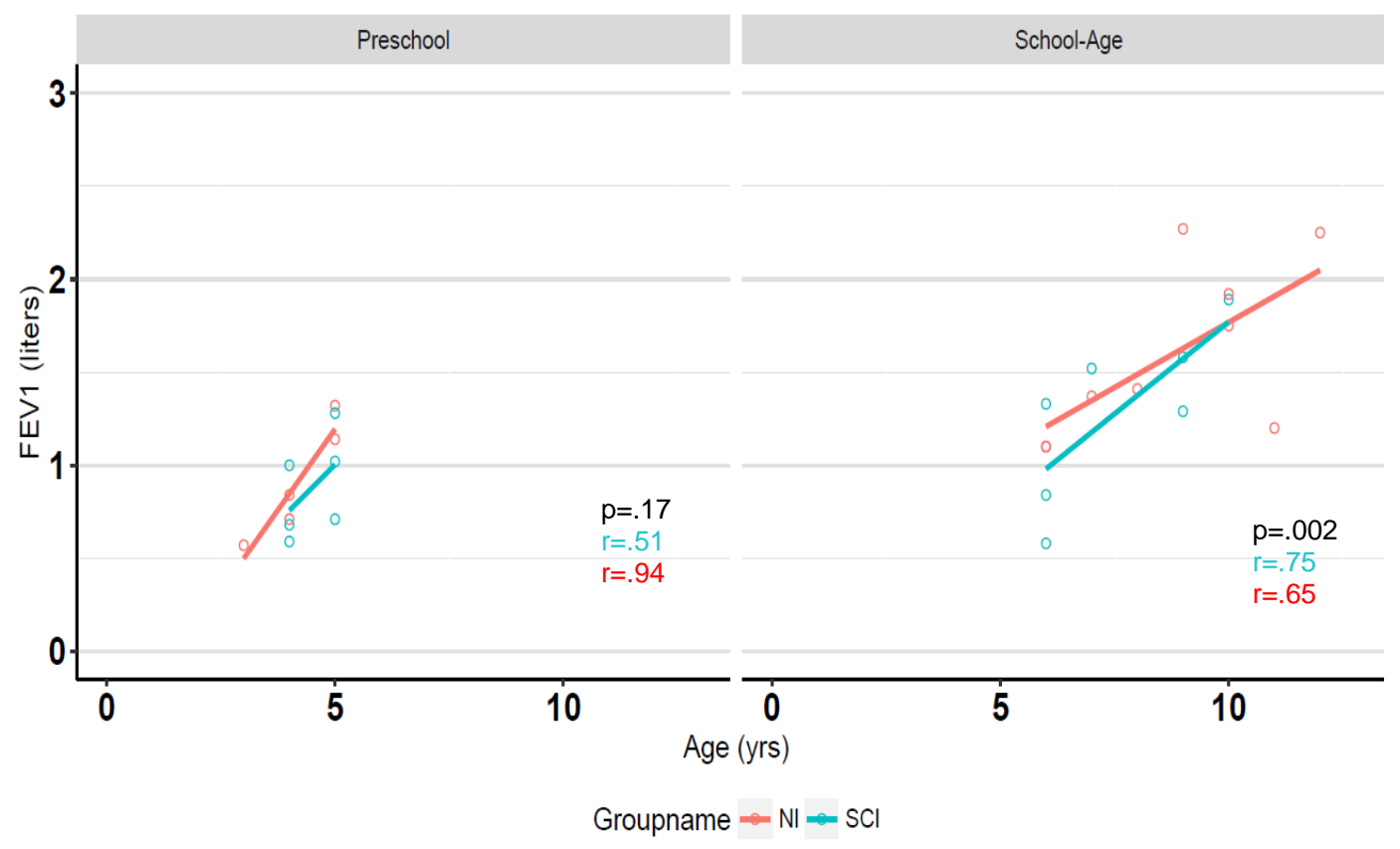

Figure VII:VI: $\mathrm{FEV}_{1}$ in TD and in children with $\mathrm{SCl}$

Correlation between Forced Expiratory Volume in one second (liters) and age (years) between non-injured and children with $\mathrm{SCl}$ age matched. A significant difference was observed between School-age $\mathrm{NI}$ and school age- SCl groups $(p=.002)$ i.e. $\mathrm{NI}$ children in school age group produced an increased volume of air in one second $\left(\mathrm{FEV}_{1}\right)$ than children with $\mathrm{SCl}$ in same age group. No significant differences were found between $\mathrm{NI}$ and $\mathrm{SCI}$ children in preschool group $(p=0.17)$. There was a linear relationship between $\mathrm{FEV}_{1}$ and age for children in both the preschool $(r=.94)$ and school age children ( $r=.65)$. However, this correlation was weaker in preschool children with $\mathrm{SCl}(r=.51)$, whereas school age children with $\mathrm{SCl}$ showed a strong relationship $(r=.75)$ between age and $\mathrm{FEV}_{1}$. 

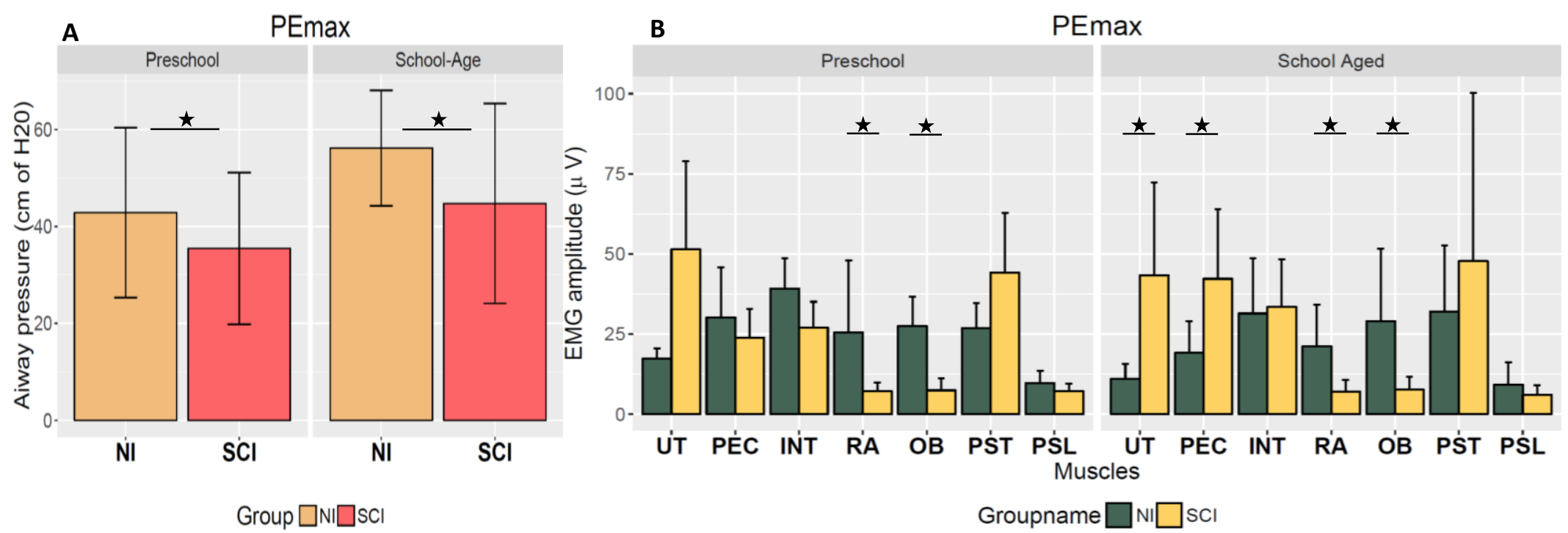

Figure VII:VII: A. PEmax and muscle activation in TD and in children with $\mathrm{SCl}$

Maximum expiratory airway pressure $\left(\mathrm{PE}_{\max }\right)$ between $\mathrm{NI}$ and $\mathrm{SCl}$ groups-age matched i.e. preschool and schoolage. In preschool age, NI children produced significantly increased PEmax airway pressure when compared to children with $\mathrm{SCI}(p=.005)$. In a school-age group, NI children produced significantly increased PEmax airway pressure compared to children with $\mathrm{SCl}(p=.0003)$ in same age group. B. Electromyography amplitude of respiratory muscles during the expiratory phase of $\mathrm{PE}_{\max }$ between $\mathrm{NI}$ and children with $\mathrm{SCl}$ for preschool and school age groups. In preschool age group, rectus abdominis (RA) and external oblique (OB) muscles activity were significantly increased in $\mathrm{NI}$ children than children with $\mathrm{SCI}(p=.006)(p=.003)$, respectively. However, no 
significant differences were observed for upper trapezius (UT) $(p=.71)$, external intercostal $($ INT) $(p=.09)$, pectoralis major (PEC) $(p=.43)$, lumbar paraspinal (PSL) $(p=.15)$, thoracic paraspinal (PST) $(p=.80)$ muscles activations. In a school-age group, rectus abdominis (RA) and external oblique $(\mathrm{OB})$ muscles activity were significantly increased in $\mathrm{NI}$ children than children with $\mathrm{SCI}(p=.03)(p=.02)$, respectively. However, children with SCI showed significantly increased activation in upper trapezius (UT) $(p=.0005)$ and pectoralis major (PEC) $(p=.008)$ than NI children. No significant differences were observed for intercostal (INT) $(p=.49)$, lumbar paraspinal (PSL) $(p=.45)$, and thoracic paraspinal PST $(p=.52)$ muscles. 

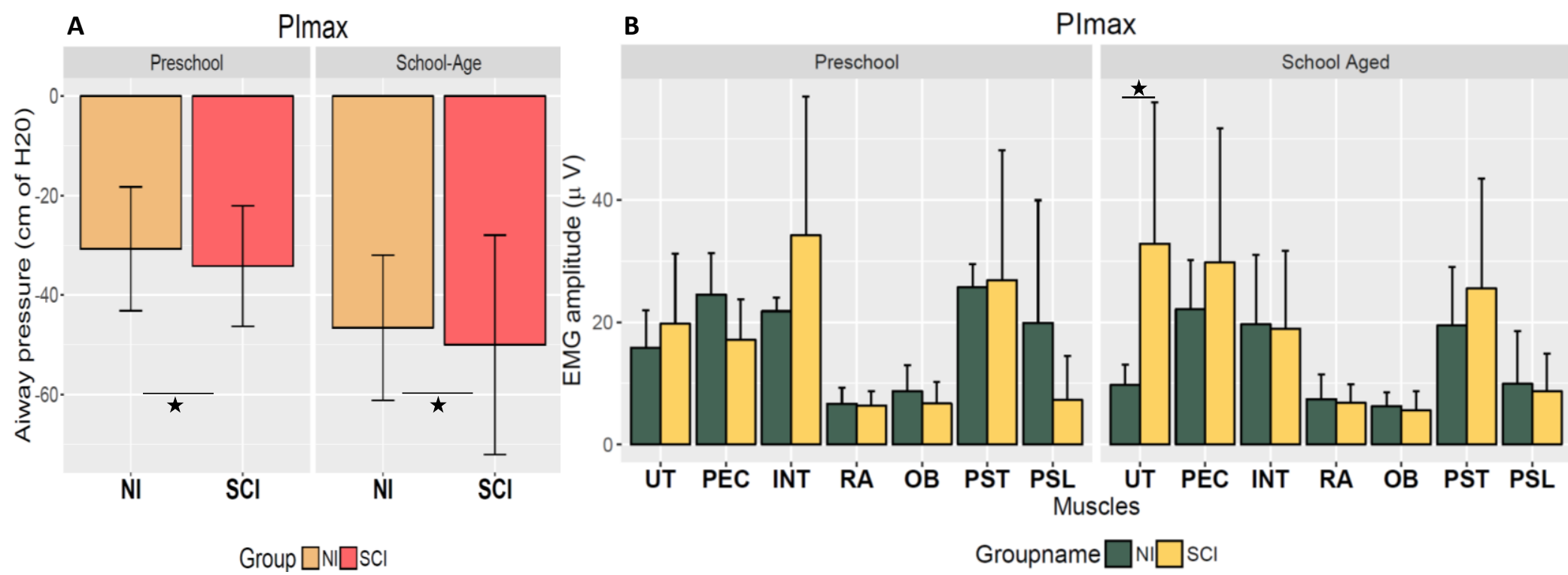

Figure VII:VIII: A. PImax and muscle activation in TD and children with SCI

Maximum inspiratory airway pressure ( $\mathrm{PI} \max )$ between $\mathrm{NI}$ and $\mathrm{SCl}$ groups-age matched i.e. preschool and schoolage. In preschool age, children with SCI produced significantly increased PImax airway pressure than NI children $(p=.01)$. In school-age group too, children with $\mathrm{SCl}$ produced significantly increased PImax airway pressure than $\mathrm{NI}$ children ( $p=.01)$. B. Electromyography amplitude of respiratory muscles during the inspiratory phase of PImax between $\mathrm{NI}$ and children with $\mathrm{SCl}$ for preschool and school age groups. In preschool age group, no significant differences were found between $\mathrm{NI}$ and children with $\mathrm{SCl}$ for any muscle i.e. INT ( $p=.06), \mathrm{OB}(p=.45), \mathrm{PEC}(p=.12)$, PSL $(p=.12)$, PST $(p=.86)$, RA ( $p=.91)$, UT ( $p=.63)$. In a school-age group, only UT muscle activation was 
significantly increased in children with $\mathrm{SCl}$ than $\mathrm{NI}$ children $(\mathrm{p}=.001)$. No significant differences were observed for any other muscle. INT ( $p=.83)$, OB ( $p=.52)$, PEC ( $p=.45)$, PSL $(p=.91)$, PST $(p=.59)$, RA ( $p=.54)$. 


\section{Discussion}

The most common abnormality reported in patients with respiratory muscle weakness is the reduction in vital capacity. Reduction in vital capacity is caused by weakness of both the inspiratory and expiratory muscles.

Neuromuscular disorders are characterized by reduced lung volumes and respiratory muscle weakness (American Thoracic Society/European Respiratory, 2002; Inal-Ince et al., 2009). We reported a significant reduction in FVC and $\mathrm{FEV}_{1}$ in school-age children with $\mathrm{SCl}$ compared to their age-matched non-injured children. PEmax values obtained were significantly impaired in both, preschool and school-age children with SCl compared to their age-matched NI children. FVC, FEV 1 \& PEmax are forced expiratory maneuvers, which require activation of abdominal muscles.

Injury to the spinal cord at or above thoracic segment results in paralysis of these expiratory muscles, resulting in decreased volume of FVC \& FEV 1 and airway pressure during $P E_{\max }$. In contrast, the parameter related to inspiratory function, PImax, was higher in children with SCI than NI children for both groups. This higher value of $\mathrm{Pl}_{\max }$ in children with $\mathrm{SCl}$ is likely due to the preservation of diaphragm muscle innervation.

Primary function of muscles is to contract and generate force. In the respiratory system, the force generated by respiratory muscles is estimated as airway pressure and contraction (shortening) as a change in lung volume or displacement of structures within chest wall (American Thoracic 
Society/European Respiratory, 2002). Measurement of maximum static expiratory $\left(P E_{\max }\right)$ and inspiratory pressures $(\mathrm{PI} \max )$ are a simple way to measure expiratory and inspiratory muscle strength, respectively. Reduced PEmax suggests severe weakness of expiratory muscles (RA \&OB), which are also involved in forced expiration during coughing and sneezing.

Results from this study confirmed the involvement/weakness of the expiratory muscles in children with $\mathrm{SCl}$. Activation of rectus abdominous and external oblique in children with $\mathrm{SCI}$ was significantly reduced during $\mathrm{PE}_{\max }$ maneuver. School-age children with SCI also exhibited higher activation of upper trapezius during $\mathrm{PE}_{\max }$ and $\mathrm{PI}_{\max }$ and pectoralis muscles during $\mathrm{PE} \mathrm{E}_{\max }$ maneuver. Both these muscles have higher innervation, suggesting greater recruitment of spared muscles above the level of the spinal injury to compensate for increased respiratory demands. However higher activation of these accessory muscles (UT \& $\mathrm{PEC}$ ) in children with $\mathrm{SCl}$ did not result in higher $\mathrm{PE}_{\max }$ air pressure compares to children in $\mathrm{NI}$ group.

Measurement of respiratory function in children with neuromuscular disorders has been recommended by ATS and it serves as a useful marker to assess the severity of disease and its prognosis over time. Our study indicates that not only these functions can be measured in TD children as young as 3 years, but also in children with SCI. However, further studies with a larger sample size need to be conducted to examine additional information in terms of sEMG changes in respiratory muscles after $\mathrm{SCl}$, effect of level and severity of injury on the development of respiratory motor control. 


\section{$\&+\$ 37(59,,, \bar{O} \& 21 \& / 86,21$}

Poor trunk control is a characteristic feature among children, adolescent and adults with neuromuscular disorders. Children with $\mathrm{SCl}$ also exhibit similar impairment in trunk control, which results in inability to sit and ambulate, as development of trunk control is an important prerequisite to develop independent sitting balance. Adults with higher cervical and thoracic SCI lack adequate trunk control to achieve functional independent sitting balance to be able to perform motor skill or ADLs. However, children who suffered SCI at early an age have not even develop that sitting balance to learn motor skills like, reaching, walking, and dressing.

In this dissertation, we assessed the segmental contribution to trunk control in TD children and compared it to children with SCI. Assessment of sitting trunk control in TD children provides valuable information, which help us to understand the impairment of trunk control in children with SCI. We measured sitting trunk control by using SATCo test and simultaneously recorded SEMG signals to measure activation of trunk muscles at each segmental level. We found that development of trunk control in TD children depends on age with 
children in older age group producing more efficient and adult like pattern of muscle activations during steady state seated position. Children in preschool (younger) group showed higher activation of trunk muscles with higher variability in muscle activation for different SATCo levels. This could be due to selection of less efficient muscle activation pattern by children in younger group. Therefore, with development, children start to use more efficient patterns with specific muscle responses (Hadders-Algra, 2010). These results are in support of neuronal group selection theory, which suggests that children in their early ages show high variability in motor response and with development; this variability is reduced as they increase their control over movements to generate more efficient motor responses.

Activation of paraspinal muscles along with sensory information about the position and movement of the body in space is necessary for the maintaining an upright sitting and standing posture. TD children in both groups, preschool and school age showed tonic activation in thoracic and lumbar paraspinal muscles. This indicates the muscle activation necessary to maintain spine stability in a neutral position and is critical in providing mechanical stability to the spine during sitting upright position. However, children with SCI higher activation in thoracic paraspinal with lower or no activation in lumbar paraspinal muscles.

Previous research in adults with $\mathrm{SCl}$ has shown that these subjects, due to impaired trunk control, compensate for the loss of postural muscle function using altered muscle synergies. $\mathrm{SCl}$ at higher thoracic level results in instability of the pelvis and lower part of the spine and to compensate for this instability, 
persons with $\mathrm{SCl}$ adopts alternative strategies for trunk control by recruiting nonpostural muscles (Cholewicki, 1997).

In our study, we found that children with $\mathrm{SCl}$ showed no significant differences in muscle activation when compared to children in TD. However, not every child with SCI was able to complete the test for all the SATCo levels so, muscle activation (sEMG) data compared only if they completed that SATCo level. In other words, the sEMG data from children with $\mathrm{SCI}$ for a specific level of SATCo support was analyzed and compared to TD children only if they were able to maintain static trunk control for that level of SATCo test. This suggests that SATCo test is reliable for measuring trunk control and is sensitive to the spinal segment being tested.

When we divided the $\mathrm{SCl}$ group into higher and lower levels of $\mathrm{SCl}$, we found significant differences between the two groups. Children with higher SCI had significantly decreased activation in muscles of lumbar segments (RA, OB \& PSL) compared to children with low SCl. Multisegmental fibers of the Erector spinae (ES) in the thoracic region, were recruited to maintain sitting upright posture in children with higher levels of $\mathrm{SCl}$, whereas children with lower levels of $\mathrm{SCl}$, due to spared innervation, used both ES at thoracic and lumbar level (Potten et al., 1999). It is also interesting to note that children who get injured at an early age are at higher risk of developing neuromuscular scoliosis (Mulcahey et al., 2013; S. Parent et al., 2011), which further leads to impaired trunk control due to imbalance between trunk muscles. Assessment of segmental deficit in children with SCI would help in designing trunk-targeted therapies; it could guide 
seating adaptations, devices with proper alignment and support and will allow gradual progression in posture control.

Trunk muscles have dual function: they are recruited during respiration and to maintain posture. In other terms, respiration and posture are linked. Diaphragm and abdominal muscles increase postural response with increase in postural demand. In non-injured adults, muscles of respiration compensate proportionately to the respiratory load. However, in patients suffering from neuromuscular diseases like $\mathrm{SCl}$, ventilation is compromised, as respiratory muscles are unable to fully overcome the resistance associated with respiration. Therefore, impaired trunk control in children with SCI can also lead to impaired breathing.

In children, $\mathrm{SCl}$ can hamper the normal development of the trunk and respiratory muscles and can potentially lead to severe respiratory insufficiency. Understandably, respiratory complications are the leading causes of death among children with SCI. Symptoms of respiratory insufficiency are highly correlated with level and severity of the spinal lesion. Injury at higher cervical and thoracic cord levels cause paralysis of muscles of respiration, which increase the workload of breathing. Considering the importance of respiratory morbidity and mortality, diagnosis of respiratory muscle weakness becomes crucial.

We evaluated spirometric lung function in children with $\mathrm{SCl}$ and compared them to their age-matched $\mathrm{NI}$ counterparts. We found that school-age children with $\mathrm{SCl}$ had decreased lung capacity $(\mathrm{FVC})$ and volume $\left(\mathrm{FEV}_{1}\right)$ compared to 
their age-matched NI. However, preschool children with SCI were not significantly different from their $\mathrm{NI}$ counterparts. To assess respiratory motor functions, first, we measured PEmax and PI $I_{\max }$ along with sEMG amplitude between preschool and school-age NI children. Older children produced increased airway pressure $\left(\mathrm{PE}_{\max } \& \mathrm{PI} \mathrm{I}_{\max }\right)$ than children in the preschool group. However, muscle activation between the groups was not significantly different.

Children with SCI produced decreased $\mathrm{PE}$ max pressure with decreased or no activation in abdominal muscles (RA \& OB). In non-injured subjects, these muscles are also used during forced expiratory maneuvers like a cough. Weak or paralyzed abdominal muscles results in a weak or an inadequate cough, leading to impaired secretion clearance, atelectasis, pneumonia and other respiratory complications (Fauroux et al., 2015; Inal-Ince et al., 2009; Khirani et al., 2014). However, Pl $\max$ was significantly higher in children with $\mathrm{SCI}$ than compared to their $\mathrm{NI}$ counterpart. This higher value of $\mathrm{PI}_{\max }$ in children with $\mathrm{SCl}$ is likely due to the preservation of diaphragm innervation.

Understanding the relationship between posture and respiratory is critical in planning effective treatment strategies to prevent further complications in children with SCl. It is well established that every muscle of the trunk participates in both, maintaining posture and respiration. Therefore, if respiratory function is compromised, the posture control maintained by trunk muscles will also be compromised. Our study suggested that both, trunk and respiratory functions are impaired in children with SCl. Therefore, early evaluation of these functions will help to design better treatment plans. There is a need for further studies to 
identify or evaluate the effectiveness of trunk targeted therapies or postural control at specific segmental level. Many clinically relevant questions could be explored; can posture and respiratory functions be improved in children with $\mathrm{SCl}$ ? What are different strategies to improve posture and respiratory control in children with SCI. In addition, sEMG studies will also help in understanding the underlying neural mechanisms of posture and respiratory and how does development impact these motor controls in children with SCI.

This dissertation offers potential to study these parameters that can lead to designing therapies and better prognosis for children with SCI. 


\section{REFERENCES}

Allen, D. D., Mulcahey, M. J., Haley, S. M., DeVivo, M. J., Vogel, L. C., McDonald, C., . . Betz, R. R. (2009). Motor Scores on the Functional Independence Measure (FIM) After Pediatric Spinal Cord Injury (SCI). Spinal Cord, 47(3), 213-217. doi:10.1038/sc.2008.94.

American Thoracic Society/European Respiratory, S. (2002). ATS/ERS Statement on respiratory muscle testing. Am J Respir Crit Care Med, 166(4), 518-624. doi:10.1164/rccm.166.4.518.

Anne Shumway-cook, M. W. (2012). Motor Control: Translating Research into Clinical Practice (Fourth ed.): Wolters Kluwer Lippincott Williams \& Wilkins.

Aslan, S. C., Chopra, M. K., McKay, W. B., Folz, R. J., \& Ovechkin, A. V. (2013). Evaluation of respiratory muscle activation using respiratory motor control assessment (RMCA) in individuals with chronic spinal cord injury. $J$ Vis $\operatorname{Exp}(77)$. doi:10.3791/50. 
Aslan, S. C., Chopra, M. K., McKay, W. B., Folz, R. J., \& Ovechkin, A. V. (2013). Evaluation of respiratory muscle activation using respiratory motor control assessment (RMCA) in individuals with chronic spinal cord injury. Journal Of Visualized Experiments: Jove(77). doi:10.3791/50178.

Basmajian Jv, D. C. (1985). Muscle Alive. Baltimore, MD: Williams \& Wilkins.

Bertenthal, B., \& Von Hofsten, C. (1998). Eye, head and trunk control: the foundation for manual development. Neurosci Biobehav Rev, 22(4), 515520.

Beydon, N., Davis, S. D., Lombardi, E., Allen, J. L., Arets, H. G. M., Aurora, P.,Wilson, N. M. (2007). An Official American Thoracic Society/European Respiratory Society Statement: Pulmonary Function Testing in Preschool Children. Am J Respir Crit Care Med, 175(12), 1304-1345. doi:10.1164/rccm.200605-642ST.

Bigongiari, A., de Andrade e Souza, F., Franciulli, P. M., Neto, S. E. R., Araujo, R. C., \& Mochizuki, L. (2011). Anticipatory and compensatory postural adjustments in sitting in children with cerebral palsy. Human Movement Science,30(3), 648-657. doi:http://dx.doi.org/10.1016/j.humov.2010.11.006 
Bjerkefors, A., Carpenter, M. G., Cresswell, A. G., \& Thorstensson, A. (2009). Trunk muscle activation in a person with clinically complete thoracic spinal cord injury. J Rehabil Med, 41(5), 390-392. doi:10.2340/16501977-0336.

Bolin, I., Bodin, P., \& Kreuter, M. (2000). Sitting position - posture and performance in C5 - C6 tetraplegia. Spinal Cord, 38(7), 425-434.

Brown, R., DiMarco, A. F., Hoit, J. D., \& Garshick, E. (2006). Respiratory dysfunction and management in spinal cord injury. Respir Care, 51(8), 853-868;discussion 869-870.

Butler, J. E. (2007). Drive to the human respiratory muscles. Respiratory Physiology \& Neurobiology, 159(2), 115-126. doi:http://dx.doi.org/10.1016/j.resp.2007.06.006.

Butler, P. B., Saavedra, S., Sofranac, M., Jarvis, S. E., \& Woollacott, M. H. (2010). Refinement, Reliability, and Validity of the Segmental Assessment of Trunk Control. Pediatric Physical Therapy, 22(3), 246-257 210.1097/PEP.1090b1013e3181e69490.

Butterworth, G., \& Cicchetti, D. (1978). Visual Calibration of Posture in Normal and Motor Retarded Down's Syndrome Infants. Perception, 7(5), 513-525. doi:10.1068/p070513. 
Cerqueira, E. P., \& Garbellini, D. (1999). Electromyographic study of the pectoralis major, serratus anterior and external oblique muscles during respiratory activity in humans. Electromyogr Clin Neurophysiol, 39(3), 131137.

Chafetz, R. S., Gaughan, J. P., Vogel, L. C., Betz, R., \& Mulcahey, M. J. (2009). The International Standards for Neurological Classification of Spinal Cord Injury: Intra-Rater Agreement of Total Motor and Sensory Scores in the Pediatric Population. The Journal Of Spinal Cord Medicine, 32(2), 157161.

Cholewicki, J., Panjabi, M. M., \& Khachatryan, A. (1997). Stabilizing function of trunk flexor-extensor muscles around a neutral spine posture. Spine (Phila Pa 1976), 22(19), 2207-2212.

Cholewicki, J., \& VanVliet Iv, J. J. (2002). Relative contribution of trunk muscles to the stability of the lumbar spine during isometric exertions. Clinical Biomechanics, 17(2), 99-105. doi:http://dx.doi.org/10.1016/S02680033(01)00118-8.

Claxton, A. R., Wong, D. T., Chung, F., \& Fehlings, M. G. (1998). Predictors of hospital mortality and mechanical ventilation in patients with cervical 
spinal cord injury. Can J Anaesth, 45(2), 144-149.

doi:10.1007/BF03013253.

College, O. (2013). Respiratory Centers of Brain. jpg. In R. C. o. Brain (Ed.), Anatomy \& Physiology, Connexions (Vol. 1.08 MB, pp. Illustration from Anatomy \& Physiology, Connexions).

Crenesse, D., Berlioz, M., Bourrier, T., \& Albertini, M. (2001). Spirometry in children aged 3 to 5 years: Reliability of forced expiratory maneuvers*. Pediatr Pulmonol, 32(1), 56-61. doi:10.1002/ppul.1089.

Cresswell, A. G., GrundstrÖM, H., \& Thorstensson, A. (1992). Observations on intra-abdominal pressure and patterns of abdominal intra-muscular activity in man. Acta Physiol Scand, 144(4), 409-418. doi:10.1111/j.17481716.1992.tb09314.x.

Curtis, D. J., Hansen, L., Luun, M., Løberg, R., Woollacott, M., Saavedra, S., . . . Bencke, J. (2015). Measuring Postural Sway in Sitting: A New Segmental Approach. Journal of Motor Behavior, 47(5), 427-435.

doi:10.1080/00222895.2014.1003782.

De Graaf-Peters V.B., B.-H. C. H., Dirks T., bakker, \& J., B. A. F., Hadders-Algra M. DEVELOPMENT OF POSTURAL CONTROL IN TYPICALLY 
DEVELOPING CHILDREN AND CHILDREN WITH CEREBRAL PALSY:

POSSIBILITIES FOR INTERVENTION? Neuroscience and Biobehavioural

Reviews (2007). doi:10.1016/j.neubiorev.2007.04.008.

De Troyer, A., Estenne, M., \& Heilporn, A. (1986). Mechanism of active expiration in tetraplegic subjects. $N$ Engl J Med, 314(12), 740-744. doi:10.1056/NEJM198603203141203.

De Troyer, A., Estenne, M., \& Vincken, W. (1986). Rib cage motion and muscle use in high tetraplegics. Am Rev Respir Dis, 133(6), 1115-1119. doi:10.1164/arrd.1986.133.6.1115.

De Troyer, A., \& Heilporn, A. (1980). Respiratory mechanics in quadriplegia. The respiratory function of the intercostal muscles. Am Rev Respir Dis, 122(4), 591-600. doi:10.1164/arrd.1980.122.4.591.

De Troyer, A., Kirkwood, P. A., \& Wilson, T. A. (2005). Respiratory Action of the Intercostal Muscles. Physiological Reviews, 85(2), 717-756. doi:10.1152/physrev.00007.2004.

De Troyer, A., Kirkwood, P. A., \& Wilson, T. A. (2005). Respiratory action of the intercostal muscles. Physiol Rev, 85(2), 717-756. doi:10.1152/physrev.00007.2004. 
De Vivo, M. J., Stuart Krause, J., \& Lammertse, D. P. (1999). Recent trends in mortality and causes of death among persons with spinal cord injury. Archives Of Physical Medicine And Rehabilitation, 80(11), 1411-1419. doi:http://dx.doi.org/10.1016/S0003-9993(99)90252-6.

Dietz, V., Colombo, G., Jensen, L., \& Baumgartner, L. (1995). Locomotor capacity of spinal cord in paraplegic patients. Ann Neurol, 37(5), 574-582. doi:10.1002/ana.410370506.

Driscoll, S. W., \& Skinner, J. (2008). Musculoskeletal Complications of Neuromuscular Disease in Children. Physical Medicine and Rehabilitation Clinics of North America, 19(1), 163-194. doi:http://dx.doi.org/10.1016/j.pmr.2007.10.003.

Eigen, H., Bieler, H., Grant, D., Christoph, K., Terrill, D., Heilman, D. K., . . . Tepper, R. S. (2001). Spirometric pulmonary function in healthy preschool children. Am J Respir Crit Care Med, 163(3 Pt 1), 619-623. doi:10.1164/ajrccm.163.3.2002054.

Estenne, M., \& De Troyer, A. (1986). The effects of tetraplegia on chest wall statics. Am Rev Respir Dis, 134(1), 121-124. doi:10.1164/arrd.1986.134.1.121. 
Estenne, M., \& Gorini, M. (1992). Action of the diaphragm during cough in tetraplegic subjects. J Appl Physiol (1985), 72(3), 1074-1080.

Estenne, M., Knoop, C., Vanvaerenbergh, J., Heilporn, A., \& De Troyer, A. (1989). The effect of pectoralis muscle training in tetraplegic subjects. Am Rev Respir Dis, 139(5), 1218-1222. doi:10.1164/ajrccm/139.5.1218.

Fauroux, B., \& Khirani, S. (2014). Neuromuscular disease and respiratory physiology in children: Putting lung function into perspective. Respirology, 19(6), 782-791. doi:10.1111/resp.12330.

Fauroux, B., Quijano-Roy, S., Desguerre, I., \& Khirani, S. (2015). THe value of respiratory muscle testing in children with neuromuscular disease. Chest, 147(2), 552-559. doi:10.1378/chest.14-0819.

Feldman, J. L., \& Del Negro, C. A. (2006). Looking for inspiration: new perspectives on respiratory rhythm. Nat Rev Neurosci, 7(3), 232-241.

Ferber-Viart, C., lonescu, E., Morlet, T., Froehlich, P., \& Dubreuil, C. (2007). Balance in healthy individuals assessed with Equitest: Maturation and normative data for children and young adults. International Journal of 
Pediatric Otorhinolaryngology, 71(7), 1041-1046.

doi:http://dx.doi.org/10.1016/j.ijporl.2007.03.012.

FH, P. (1917). The all or none principle in graded response of skeletal muscle. American Journal of Physiology, 44, 517-542.

Field, D., \& Livingstone, R. (2013). Clinical tools that measure sitting posture, seated postural control or functional abilities in children with motor impairments: a systematic review. Clin Rehabil, 27(11), 994-1004. doi:10.1177/0269215513488122.

Finkel, R. S., Weiner, D. J., Mayer, O. H., McDonough, J. M., \& Panitch, H. B. (2014). Respiratory muscle function in infants with spinal muscular atrophy type I. Pediatr Pulmonol, 49(12), 1234-1242. doi:10.1002/ppul.22997

Fishburn, M. J., Marino, R. J., \& Ditunno, J. F., Jr. (1990). Atelectasis and pneumonia in acute spinal cord injury. Arch Phys Med Rehabil, 71(3), 197200.

Forssberg, H., \& Hirschfeld, H. (1994). Postural adjustments in sitting humans following external perturbations: muscle activity and kinematics. Exp Brain Res, 97(3), 515-527. doi:10.1007/bf00241545. 
Forssberg, H., \& Nashner, L. (1982). Ontogenetic development of postural control in man: adaptation to altered support and visual conditions during stance. The Journal of Neuroscience, 2(5), 545-552.

Foudriat, B. A., Di Fabio, R. P., \& Anderson, J. H. (1993). Sensory organization of balance responses in children 3-6 years of age: a normative study with diagnostic implications. International Journal of Pediatric Otorhinolaryngology, 27(3), 255-271. doi:http://dx.doi.org/10.1016/01655876(93)90231-Q.

França, D. C., Camargos, P. A. M., Jones, M. H., Martins, J. A., Vieira, B. d. S. P. P., Colosimo, E. A., . . Parreira, V. F. (2016). Prediction equations for spirometry in four- to six-year-old children. J Pediatr (Rio J), 92(4), 400408. doi:http://dx.doi.org/10.1016/j.jped.2015.10.005.

Frank, J. S., \& Earl, M. (1990). Coordination of posture and movement. Phys Ther, 70(12), 855-863.

Fujiwara, T., Hara, Y., \& Chino, N. (1999). Expiratory function in complete tetraplegics: study of spirometry, maximal expiratory pressure, and muscle activity of pectoralis major and latissimus dorsi muscles. Am J Phys Med Rehabil, 78(5), 464-469. 
Gary Kamen, D. G. (2010). Essentials of Electromyography (1st ed.): Human Kinetics.

Ghez, C., Hening, W., \& Gordon, J. (1991). Organization of voluntary movement. Curr Opin Neurobiol, 1(4), 664-671.

Gray, H. (1918). Anatomy of the Human Body Myology (pp. 1). Retrieved from http://www.bartleby.com/107/121.html .

Gross, D., Grassino, A., Ross, W. R., \& Macklem, P. T. (1979). Electromyogram pattern of diaphragmatic fatigue. J Appl Physiol Respir Environ Exerc Physiol, 46(1), 1-7.

Gutierrez A, S. A. (2006). Electromyography in Neurorehbilitation [Press release].

Hadders-Algra, M. (2000). The Neuronal Group Selection Theory: a framework to explain variation in normal motor development. Developmental Medicine \& Child Neurology, 42(8), 566-572. doi:10.1111/j.14698749.2000.tb00714.x.

Hadders-Algra, M. (2010). Variation and Variability: Key Words in Human Motor Development. Phys Ther, 90(12), 1823-1837. doi:10.2522/ptj.20100006 
Harbourne, R. T., \& Stergiou, N. (2003). Nonlinear analysis of the development of sitting postural control. Developmental Psychobiology, 42(4), 368-377. doi:10.1002/dev.10110.

Harkema, S., Gerasimenko, Y., Hodes, J., Burdick, J., Angeli, C., Chen, Y., . . . Edgerton, V. R. (2011). Effect of epidural stimulation of the lumbosacral spinal cord on voluntary movement, standing, and assisted stepping after motor complete paraplegia: a case study. Lancet, 377(9781), 1938-1947. doi:10.1016/S0140-6736(11)60547-3.

Hedberg, Å., Carlberg, E. B., Forssberg, H., \& Algra, M. H. (2005). Development of postural adjustments in sitting position during the first half year of life. Developmental Medicine \& Child Neurology, 47(5), 312-320. doi:10.1111/j.1469-8749.2005.tb01142.x.

Hedberg, Å., Forssberg, H., \& Hadders-Algra, M. (2004). Postural adjustments due to external perturbations during sitting in 1-month-old infants: evidence for the innate origin of direction specificity. Exp Brain Res, 157(1), 10-17. doi:10.1007/s00221-003-1811-z.

Hedberg, Å., Schmitz, C., Forssberg, H., \& Hadders-Algra, M. (2007). Early development of postural adjustments in standing with and without support. Exp Brain Res, 178(4), 439-449. doi:10.1007/s00221-006-0754-6. 
Hershenson, M. B., Stark, A. R., \& Mead, J. (1989). Action of the inspiratory muscles of the rib cage during breathing in newborns. Am Rev Respir Dis, 139(5), 1207-1212. doi:10.1164/ajrccm/139.5.1207.

Hirschfeld, H., \& Forssberg, H. (1991). Phase-dependent modulations of anticipatory postural activity during human locomotion. $J$ Neurophysiol, 66(1), 12-19.

Hirschfeld, H., \& Forssberg, H. (1992). Development of anticipatory postural adjustments during locomotion in children. J Neurophysiol, 68(2), 542-550.

Hodges, P. W., \& Gandevia, S. C. (2000). Activation of the human diaphragm during a repetitive postural task. J Physiol, 522(Pt 1), 165-175. doi:10.1111/j.1469-7793.2000.t01-1-00165.xm.

Hodges, P. W., Gurfinkel, V. S., Brumagne, S., Smith, T. C., \& Cordo, P. C. (2002). Coexistence of stability and mobility in postural control: evidence from postural compensation for respiration. Exp Brain Res, 144(3), 293302. doi:10.1007/s00221-002-1040-x. 
Horak, F. B., Nashner, L. M., \& Diener, H. C. (1990). Postural strategies associated with somatosensory and vestibular loss. Exp Brain Res, 82(1), 167-177.

Iglewicz B, H. D. (1993). How to detect and handle outliers [Press release].

Inal-Ince, D., Savci, S., Arikan, H., Saglam, M., Vardar-Yagli, N., Bosnak-Guclu, M., \& Dogru, D. (2009). Effects of scoliosis on respiratory muscle strength in patients with neuromuscular disorders. The Spine Journal, 9(12), 981 986. doi:http://dx.doi.org/10.1016/j.spinee.2009.08.451.

Ito, K., Nonaka, K., Ogaya, S., Ogi, A., Matsunaka, C., \& Horie, J. (2016). Surface electromyography activity of the rectus abdominis, internal oblique, and external oblique muscles during forced expiration in healthy adults. Journal of Electromyography and Kinesiology, 28, 76-81. doi:http://dx.doi.org/10.1016/j.jelekin.2016.03.007.

Jackson, A. B., \& Groomes, T. E. (1994). Incidence of respiratory complications following spinal cord injury. Arch Phys Med Rehabil, 75(3), 270-275.

Jain, N. B., Brown, R., Tun, C. G., Gagnon, D., \& Garshick, E. (2006). Determinants of Forced Expiratory Volume in 1 Second (FEV1), Forced Vital Capacity (FVC), and FEV1/FVC in Chronic Spinal Cord Injury. 
Archives Of Physical Medicine And Rehabilitation, 87(10), 1327-1333. doi:http://dx.doi.org/10.1016/j.apmr.2006.06.015.

Kanengiser, S., \& Dozor, A. J. (1994). Forced expiratory maneuvers in children aged 3 to 5 years. Pediatr Pulmonol, 18(3), 144-149. doi:10.1002/ppul.1950180305.

Kenyon, L. K., \& Blackinton, M. T. (2011). Applying Motor-Control Theory to Physical Therapy Practice: A Case Report. Physiotherapy Canada, 63(3), 345-354. doi:10.3138/ptc.2010-06.

Khirani, S., Ramirez, A., Aubertin, G., Boulé, M., Chemouny, C., Forin, V., \& Fauroux, B. (2014). Respiratory muscle decline in duchenne muscular dystrophy. Pediatr Pulmonol, 49(5), 473-481. doi:10.1002/ppul.22847.

Kirshblum, S. C., Burns, S. P., Biering-Sorensen, F., Donovan, W., Graves, D. E., Jha, A., . . Waring, W. (2011). International standards for neurological classification of spinal cord injury (revised 2011). J Spinal Cord Med, 34(6), 535-546. doi:10.1179/204577211X13207446293695.

Lancourt, J. E., Dickson, J. H., \& Carter, R. E. (1981). Paralytic spinal deformity following traumatic spinal-cord injury in children and adolescents. $J$ Bone Joint Surg Am, 63(1), 47-53. 
Lane, M. A. (2011). Spinal respiratory motoneurons and interneurons. Respir Physiol Neurobiol, 179(1), 3-13. doi:10.1016/j.resp.2011.07.004.

Legrand, A., Schneider, E., Gevenois, P. A., \& De Troyer, A. (2003). Respiratory effects of the scalene and sternomastoid muscles in humans. J Appl Physiol (1985), 94(4), 1467-1472. doi:10.1152/japplphysiol.00869.2002.

Lemons, V. R., \& Wagner, F. C., Jr. (1994). Respiratory complications after cervical spinal cord injury. Spine (Phila Pa 1976), 19(20), 2315-2320.

Leung, A. N., Bull, T. M., Jaeschke, R., Lockwood, C. J., Boiselle, P. M., Hurwitz, L. M., ... Pregnancy, A. S. C. o. P. E. i. (2012). American Thoracic Society documents: an official American Thoracic Society/Society of Thoracic Radiology Clinical Practice Guideline--Evaluation of Suspected Pulmonary Embolism in Pregnancy. Radiology, 262(2), 635-646. doi:10.1148/radiol.11114045.

Levizky, M. G. (1995). Pulmonary Physiology (4th ed.): McGRAW-HILL, INC.

Lim, H. K., Lee, D. C., McKay, W. B., Priebe, M. M., Holmes, S. A., \& Sherwood, 
A. M. (2005). Neurophysiological assessment of lower-limb voluntary control in incomplete spinal cord injury. Spinal Cord, 43(5), 283-290. doi:10.1038/sj.sc.3101679.

M.R. Miller, J. H., V. Brusasco, F. Burgos, R. Casaburi, A. Coates, R. Crapo, P. Enright, C.P.M. van der Grinten, P. Gustafsson, R. Jensen, D.C. Johnson, N. Maclntyre, R. McKay, D. Navajas, O.F. Pedersen, R. Pellegrino, G. Viegi and J. Wanger. (2005). ATS/ERS TASK FORCE: STANDARDISATION OF LUNG FUNCTION TESTING. Eur Respir J, 26, 319-338. doi:10.1183/09031936.05.00034805.

Mansel, J. K., \& Norman, J. R. (1990). Respiratory complications and management of spinal cord injuries. Chest, 97(6), 1446-1452.

Massion, J. (1992). Movement, posture and equilibrium: Interaction and coordination. Prog Neurobiol, 38(1), 35-56. doi:http://dx.doi.org/10.1016/0301-0082(92)90034-C.

Massion, J. (1992). Movement, posture and equilibrium: interaction and coordination. Prog Neurobiol, 38(1), 35-56. 
Massion, J. (1998). Postural Control Systems in Developmental Perspective. Neuroscience \& Biobehavioral Reviews, 22(4), 465-472. doi:http://dx.doi.org/10.1016/S0149-7634(97)00031-6.

Mayfield, J. K., Erkkila, J. C., \& Winter, R. B. (1981). Spine deformity subsequent to acquired childhood spinal cord injury. J Bone Joint Surg Am, 63(9), $1401-1411$.

MB, M. (1943). The neuromuscular maturation of the human infant [Press release].

McGill, S. M., Grenier, S., Kavcic, N., \& Cholewicki, J. (2003). Coordination of muscle activity to assure stability of the lumbar spine. Journal of Electromyography and Kinesiology, 13(4), 353-359. doi:http://dx.doi.org/10.1016/S1050-6411(03)00043-9.

Meekins, G. D., So, Y., \& Quan, D. (2008). American Association of Neuromuscular \& Electrodiagnostic Medicine evidenced-based review: use of surface electromyography in the diagnosis and study of neuromuscular disorders. Muscle Nerve, 38(4), 1219-1224. doi:10.1002/mus.21055. 
Mehta, S., Betz, R., Mulcahey, M. J., McDonald, C., \& Vogel, L. (2004). Effect of Bracing on Paralytic Scoliosis Secondary to Spinal Cord Injury. The Journal Of Spinal Cord Medicine, 27(sup1), S88-S92. doi:10.1080/10790268.2004.11753448.

Merletti, R., Rainoldi, A., \& Farina, D. (2001). Surface Electromyography for Noninvasive Characterization of Muscle. Exercise and Sport Sciences Reviews, 29(1), 20-25.

Miller, M. R., Hankinson, J., Brusasco, V., Burgos, F., Casaburi, R., Coates, A., . .. Force, A. E. T. (2005). Standardisation of spirometry. Eur Respir J, 26(2), 319-338. doi:10.1183/09031936.05.00034805.

Mittelstaedt, H. (1964). Basic control patterns of orientational homeostasis. Symp Soc Exp Biol, 18, 365-385.

Mittelstaedt, H., \& Fricke, E. (1988). The relative effect of saccular and somatosensory information on spatial perception and control. Adv Otorhinolaryngol, 42, 24-30.

Mulcahey, M. J., Gaughan, J. P., Betz, R. R., Samdani, A. F., Barakat, N., \& Hunter, L. N. (2013). Neuromuscular scoliosis in children with spinal cord injury. Top Spinal Cord Inj Rehabil, 19(2), 96-103. doi:10.1310/sci1902-96 
Nashner LM, M. G. (1985). The organization of human postural movements: a formal basis and experimental synthesis. Behav Brain, 8, 135-172.

Nicot, F., Hart, N., Forin, V., Boulé, M., Clément, A., Polkey, M. I., . . Fauroux, B. (2006). Respiratory Muscle Testing. Am J Respir Crit Care Med, 174(1), 67-74. doi:10.1164/rccm.200512-18410C.

Nobre, M. E., Lopes, F., Cordeiro, L., Marinho, P. E., Silva, T. N., Amorim, C., Dornelas de Andrade, A. (2007). Inspiratory muscle endurance testing: pulmonary ventilation and electromyographic analysis. Respir Physiol Neurobiol, 155(1), 41-48. doi:10.1016/j.resp.2006.04.005.

NSCISC. (2016). Spinal Cord Injury (SCI) Facts and Figures at a Glance. National Spinal Cord Injury Statistical Center.

Openshaw, P., Edwards, S., \& Helms, P. (1984). Changes in rib cage geometry during childhood. Thorax, 39(8), 624-627.

Ovechkin, A., Vitaz, T., de Paleville, D. T., Aslan, S., \& McKay, W. (2010). Evaluation of respiratory muscle activation in individuals with chronic spinal cord injury. Respir Physiol Neurobiol, 173(2), 171-178. doi:10.1016/j.resp.2010.07.013. 
Ovechkin, A. V., Vitaz, T. W., Terson de Paleville, D. G. L., \& McKay, W. B. (2013). Quality of Residual Neuromuscular Control and Functional Deficits in Patients with Spinal Cord Injury. Front Neurol, 4, 174. doi:10.3389/fneur.2013.00174.

Parent, S., Dimar, J., Dekutoski, M., \& Roy-Beaudry, M. (2010). Unique features of pediatric spinal cord injury. Spine (Phila Pa 1976), 35(21 Suppl), S202208. doi:10.1097/BRS.0b013e3181f35acb.

Parent, S., Mac-Thiong, J.-M., Roy-Beaudry, M., Sosa, J. F., \& Labelle, H. (2011). Spinal Cord Injury in the Pediatric Population: A Systematic Review of the Literature. J Neurotrauma, 28(8), 1515-1524. doi:10.1089/neu.2009.1153.

Parent, S., Mac-Thiong, J. M., Roy-Beaudry, M., Sosa, J. F., \& Labelle, H. (2011). Spinal cord injury in the pediatric population: a systematic review of the literature. J Neurotrauma, 28(8), 1515-1524. doi:10.1089/neu.2009.1153.

Pattinson, K. T., Governo, R. J., Maclntosh, B. J., Russell, E. C., Corfield, D. R., Tracey, I., \& Wise, R. G. (2009). Opioids depress cortical centers 
responsible for the volitional control of respiration. J Neurosci, 29(25), 8177-8186. doi:10.1523/JNEUROSCI.1375-09.2009.

Pattinson, K. T., Mitsis, G. D., Harvey, A. K., Jbabdi, S., Dirckx, S., Mayhew, S. D., . . Wise, R. G. (2009). Determination of the human brainstem respiratory control network and its cortical connections in vivo using functional and structural imaging. Neuroimage, 44(2), 295-305. doi:10.1016/j.neuroimage.2008.09.007.

Potten, Y. J. M., Seelen, H. A. M., Drukker, J., Reulen, J. P. H., \& Drost, M. R. (1999). Postural muscle responses in the spinal cord injured persons during forward reaching. Ergonomics, 42(9), 1200-1215. doi:10.1080/001401399185081.

Powell, A., \& Davidson, L. (2015). Pediatric Spinal Cord Injury: A Review by Organ System. Physical Medicine and Rehabilitation Clinics of North America, 26(1), 109-132. doi:http://dx.doi.org/10.1016/j.pmr.2014.09.002.

Rachwani, J., Santamaria, V., Saavedra, S. L., Wood, S., Porter, F., \& Woollacott, M. H. (2013). Segmental trunk control acquisition and reaching in typically developing infants. Exp Brain Res, 228(1), 131-139. doi:10.1007/s00221-013-3544-y. 
Ratnovsky, A., Elad, D., \& Halpern, P. (2008). Mechanics of respiratory muscles. Respiratory Physiology \& Neurobiology, 163(1-3), 82-89. doi:http://dx.doi.org/10.1016/j.resp.2008.04.019.

Roth, E. J., Lu, A., Primack, S., Oken, J., Nusshaum, S., Berkowitz, M., \& Powley, S. (1997). Ventilatory function in cervical and high thoracic spinal cord injury. Relationship to level of injury and tone. Am J Phys Med Rehabil, 76(4), 262-267.

RStudio. (2012). R Studio (Version 3.3.0).

Saavedra, S. L., \& Woollacott, M. H. (2015). Segmental Contributions to Trunk Control in Children With Moderate-to-Severe Cerebral Palsy. Archives Of Physical Medicine And Rehabilitation, 96(6), 1088-1097. doi:http://dx.doi.org/10.1016/j.apmr.2015.01.016.

Sandra L. Saavedra, P. v. D. a. M. H. W. (2012). Learning about gravity: segmental assessment of upright control as infants develop independent sitting. J Neurophysiology. doi:10.1152/jn.01193.2011.

Schilero, G. J., Spungen, A. M., Bauman, W. A., Radulovic, M., \& Lesser, M. (2009). Pulmonary function and spinal cord injury. Respir Physiol Neurobiol, 166(3), 129-141. doi:10.1016/j.resp.2009.04.002. 
Schmidt, R. (2011). Motor Control and Learning Human Kinetics

Schmitt, J., Midha, M., \& McKenzie, N. (1991). Medical complications of spinal cord disease. Neurol Clin, 9(3), 779-795.

Schottler, J., Vogel, L. C., \& Sturm, P. (2012). Spinal cord injuries in young children: a review of children injured at 5 years of age and younger. Dev Med Child Neurol, 54(12), 1138-1143. doi:10.1111/j.14698749.2012.04411.x.

Seelen, H. A. M., Janssen-Potten, Y. J. M., \& Adam, J. J. (2001). Motor preparation in postural control in seated spinal cord injured people. Ergonomics, 44(4), 457-472. doi:10.1080/00140130010020549.

Seevedra. (2010). Contributional of spinal segments to control posture during typical and atypical development.

Sharma, S., Wu, C., Andersen, T., Wang, Y., Hansen, E. S., \& Bünger, C. E. (2013). Prevalence of complications in neuromuscular scoliosis surgery: a literature meta-analysis from the past 15 years. European Spine Journal, 22(6), 1230-1249. doi:10.1007/s00586-012-2542-2. 
Shavelle, R. M., DeVivo, M. J., Paculdo, D. R., Vogel, L. C., \& Strauss, D. J. (2007). Long-Term Survival After Childhood Spinal Cord Injury. The Journal Of Spinal Cord Medicine, 30(Suppl 1), S48-S54.

Sherwood, A. M., Graves, D. E., \& Priebe, M. M. (2000). Altered motor control and spasticity after spinal cord injury: subjective and objective assessment. Journal of Rehabilitation Research \& Development, 37(1), 41-52.

Sherwood, A. M., McKay, W. B., \& Dimitrijevic, M. R. (1996). Motor control after spinal cord injury: assessment using surface EMG. Muscle Nerve, 19(8), 966-979. doi:10.1002/(SICI)1097-4598(199608)19:8<966::AIDMUS5>3.0.CO;2-6.

Smith, J. C., Abdala, A. P. L., Borgmann, A., Rybak, I. A., \& Paton, J. F. R. (2013). Brainstem respiratory networks: building blocks and microcircuits. Trends Neurosci, 36(3), 152-162. doi:http://dx.doi.org/10.1016/j.tins.2012.11.004.

Smith, J. C., Abdala, A. P. L., Rybak, I. A., \& Paton, J. F. R. (2009). Structural and functional architecture of respiratory networks in the mammalian brainstem. Philosophical Transactions of the Royal Society B: Biological Sciences, 364(1529), 2577-2587. doi:10.1098/rstb.2009.0081. 
Terson de Paleville, D., \& Lorenz, D. (2015). Compensatory muscle activation during forced respiratory tasks in individuals with chronic spinal cord injury. Respiratory Physiology \& Neurobiology, 217, 54-62. doi:http://dx.doi.org/10.1016/j.resp.2015.07.001.

Ting, L. H., \& McKay, J. L. (2007). Neuromechanics of muscle synergies for posture and movement. Current Opinion in Neurobiology, 17(6), 622-628. doi:http://dx.doi.org/10.1016/j.conb.2008.01.002.

Tomalak, W., Pogorzelski, A., \& Prusak, J. (2002). Normal values for maximal static inspiratory and expiratory pressures in healthy children. Pediatr Pulmonol, 34(1), 42-46. doi:10.1002/ppul.10130.

van den Berg, M. E. L., Castellote, J. M., de Pedro-Cuesta, J., \& MahilloFernandez, I. (2010). Survival after Spinal Cord Injury: A Systematic Review. J Neurotrauma, 27(8), 1517-1528. doi:10.1089/neu.2009.1138.

Van der Fits, I. B., Otten, E., Klip, A. W., Van Eykern, L. A., \& Hadders-Algra, M. (1999). The development of postural adjustments during reaching in 6 - to 18-month-old infants. Evidence for two transitions. Exp Brain Res, 126(4), 517-528. 
van Silfhout, L., Peters, A. E. J., Berlowitz, D. J., Schembri, R., Thijssen, D., \& Graco, M. (2016). Long-term change in respiratory function following spinal cord injury. Spinal Cord, 54(9), 714-719. doi:10.1038/sc.2015.233.

Vilozni, D., Barker, M., Jellouschek, H., Heimann, G., \& Blau, H. (2001). An Interactive Computer-Animated System (SpiroGame) Facilitates Spirometry in Preschool Children. Am J Respir Crit Care Med, 164(12), 2200-2205. doi:10.1164/ajrccm.164.12.2101002.

Vinit, S., \& Kastner, A. (2009). Descending bulbospinal pathways and recovery of respiratory motor function following spinal cord injury. Respir Physiol Neurobiol, 169(2), 115-122. doi:10.1016/j.resp.2009.08.004.

Warren, P. M., Awad, B. I., \& Alilain, W. J. (2014). Drawing breath without the command of effectors: the control of respiration following spinal cord injury. Respir Physiol Neurobiol, 203, 98-108. doi:10.1016/j.resp.2014.08.005.

Wilson, S. H., Cooke, N. T., Edwards, R. H., \& Spiro, S. G. (1984). Predicted normal values for maximal respiratory pressures in caucasian adults and children. Thorax, 39(7), 535-538. doi:10.1136/thx.39.7.535. 
Wilson, T. A., Legrand, A., Gevenois, P.-A., \& De Troyer, A. (2001). Respiratory effects of the external and internal intercostal muscles in humans. $J$ Physiol, 530(Pt 2), 319-330. doi:10.1111/j.1469-7793.2001.0319l.x.

Woollacott, M., Debu, B., \& Mowatt, M. (1987). Neuromuscular control of posture in the infant and child: is vision dominant? J Mot Behav, 19(2), 167-186.

Woollacott, M. H., \& Burtner, P. (1996). Neural and musculoskeletal contributions to the development of stance balance control in typical children and in children with cerebral palsy. Acta Paediatr Suppl, 416, 58-62.

Yokoba, M., Abe, T., Katagiri, M., Tomita, T., \& Easton, P. A. (2003). Respiratory muscle electromyogram and mouth pressure during isometric contraction. Respir Physiol Neurobiol, 137(1), 51-60.

Zaba, R. (2002). [A twenty-year research on the pathogenesis of functional disorders of the respiratory tract in children and adolescents with idiopathic scoliosis]. Wiad Lek, 55 Suppl 1(Pt 2), 998-1002.

Zaba, R. (2003a). Peak expiratory flow in children and adolescents with idiopathic scoliosis. Wiad Lek, 56(11-12), 552-555. 
Zaba, R. (2003b). Pulmonary compensatory indexes in children and adolescents with idiopathic scoliosis I degree. Wiad Lek, 56(5-6), 250-253.

Zimmer, M. B., Nantwi, K., \& Goshgarian, H. G. (2008). Effect of spinal cord injury on the neural regulation of respiratory function. Exp Neurol, 209(2), 399-406. doi:10.1016/j.expneurol.2007.05.015. 


\section{APPENDIX:}

\section{ABBREVIATIONS}

TD- Typical developing

SCl- Spinal cord injury

SATCo- Segmental assessment of trunk control

SSC-Shoulder static control

ASC- Axilla static control

ISSC- Inferior scapula static control

OLRSC- Over lower ribs static control

BRSC- Below ribs static control

PSC- Pelvic static control

NSSC- No support static control

sEMG- Surface electromyography

ADLs- Activities of daily living

TMC- Trunk motor control

RMC- Respiratory motor control

RMCA- Respiratory motor control assessment

FVC- Forced Vital Capacity

FEV ${ }_{1}$ - Forced Expiratory Volume in one second 


\section{INFORMED CONSENT:}

$\begin{array}{ll} & \text { Uofl. Institutional Review Boards } \\ \text { IRB NUMBER: } 15.0585 & \text { IRB APPROVAL DATE: 07/26/2016 } \\ \text { IRdvancing a New Trajectory of Outcomes for Children with Paralysis through Activity-Based Rehabilitation } & \text { IRB EXPIRATION DATE: 07/25/2017 }\end{array}$

Advancing a New Trajectory of Outco

IRB EXPIRATION DATE: 07/25/2017

INFORMED CONSENT DOCUMENT AND RESEARCH AUTHORIZATION

\section{Advancing a New Trajectory of Outcomes for Children with Paralysis} through Activity-Based Rehabilitation

Grants number:

Sponsor(s) name \& address:

Investigator(s) name, Degree, University Department, \& address:

Site(s) where study is to be conducted:

Phone number for subjects to call for questions:
OGMB141540 (Kosair Charities)

G2596 (Helmsley)

The Leona M. \& Harry B. Helmsley Charitable Trust 230 Park Avenue, Suite 659

New York, NY 10169

Kosair Charities

982 Eastern Parkway, P.O. Box 37370

Louisville, KY 40217

Andrea Behrman, PhD, PT

Frazier Rehab Institute, Suite 1506

220 Abraham Flexner Way

Louisville, KY 40202

Frazier Rehabilitation Institute $\mathrm{U}$ of L Department of Neurological Surgery

$502-581-8675$

Introduction and Background Information

Your child is invited to take part in a research study because your child has an injury or disease of the spinal cord that affects your child's nerves and muscles and impairs your child's ability to move, sit, stand, and/or walk and your child is currently under 18 years of age, and has previously participated in activity based therapy (ABT) at Frazier Rehab Institute, Louisville, KY. The study is being conducted under the direction of Andrea L. Behrman, PhD, PT and colleagues at the University of Louisville. About 50 subjects will be invited to take part in this research study.

\section{Purpose}

Currently there is no longitudinal data documenting the effects of ABT on the function of children with $\mathrm{SCl}$ and their quality of life. The purpose of this study is to track outcome measures overtime to see if ABT, such as Locomotor Training (LT), has an immediate and long-term effect on function, health, healthcare utilization, and quality of life in children with $\mathrm{SCl}$ and their caregivers (e.g. parents).

If you agree to participate in this study, you are agreeing to share clinical, research, health-related, and quality of life information with us until your child reaches the age of 18 . Your family's participation may last up to 18 years.

\section{Procedures}

This is a longitudinal study - that means we will collect information from your child over time until your child turn 18. Much of this information may be provided and learned during your child's annual clinical visit at Frazier Rehabilitation Institute, Louisville, $\mathrm{KY}$. 
Andrea L. Behrman PhD, PT, FAPTA

During your child's annual clinical visit we will assess your child's physical abilities, function, and quality of life similarly each time. Should your child miss a return visit, we would still access information from your child's medical records, questionnaires, and interview with your child conducted over the phone or via email. We will ask that your child or you share information with us from your child's routine doctor visits to check for scoliosis or possible hip dislocation (position of your child's leg bone in the socket)

We also would like to communicate with your child on a semi-annual basis to update any new information related to your child's injury and use of health-care resources, for instance any doctor visits, hospitalizations, medications. Specifically, we will collect the following information:

1. Pediatric Physical Therapy Exam

2. Spine and Hip Assessment

3. Quality of Life and Participation

4. Healthcare Use

1. The Pediatric Physical Therapy - Clinical Evaluation: As part of clinical evaluation, your child will receive the following assessments and results maintained in our database:

PEDSQL: This is a tool to determine how the injury affects your child's quality of life. There are two components to the PEDsQL, the child self-report and the parent proxy report. It is possible that you and/or your child may be videotaped.

Pain Assessment: We will ask your child to tell us when they have pain and how much pain they are having The way we ask depends on your child's age. Three different scales are used and the appropriate one will be selected for your child based on your child's age.

Growth and Health Statistics: We will measure your child's height, weight, and vital signs in sitting, standing, and lying positions (BP, HR, Oxygen saturation (how much oxygen your child is getting into their bloodstream).

Orthostatic Hypotension Test: The goal of this assessment is to identify children who have orthostatic hypotension (a change in your child's blood pressure based on the position that they are in - whether sitting, standing, or lying down). The blood pressure, heart rate, and time of day are recorded for each child during a 5 minute supine resting period and then in response to an immediate position change from supine to sitting that lasts 3 minutes. After 3 minutes of sitting up at 90 degrees, blood pressure, heart rate, and time of day are recorded once again.

Clonus: This assessment is used to determine the presence of clonus (rapid alternating beat of the ankle) in each child.

Babinski Test: This reflex test is used to test the integrity of the nervous system in children after the age of 1 . Each child will have a pointed object (usually the blunt end of a reflex hammer) stroked across the bottom of their foot, while in the seated position. The stroke should initiate a response that will be recorded

Tendon Reflex: Tendon taps are performed at the Patellar (knee) and Achilles (ankle) tendon to observe the responsiveness of reflexes.

Orthopedic Measures: These measures assess range of motion, growth, alignment, and skeletal development across time.

Timed Sit: The goal is to measure improvements in sitting time and endurance with and without assistance while maintaining optimal sitting and trunk alignment. 
Timed Stand: This is an assessment of ability to maintain standing over ground without physical assistance of another person, but allowing for use of an assistive device as required. The test measures improvements in standing time and endurance.

Pediatric Balance Scale: This scale measures balance in various positions and while performing functional activities. There are 14 different challenges tested and scored $(0-4)$ as part of this assessment.

Pediatric Neuromuscular Recovery Scale (NRS):

- This test has your child perform every day activities like sitting up from lying down, sitting, reaching, grasping, holding objects, lifting an object, standing up, standing, and walking. Your child will be asked to do these without help from others and without use of devices to assist your child.

- Eight of these tasks are done while on a mat or bench or on the ground while three of the items are done on a treadmill. While on the treadmill, your child will wear a modified rock-climbing harness that is connected to an overhead support to help hold your child up.

- A safety cable is also attached to the harness in case your child lose their balance or begin to fall during any of the treadmill activities. Therapists or rehab technicians will help your child throughout this test to be sure your child is safe and understand the instructions.

- This test takes about 45 minutes to complete.

Segmental Assessment of Trunk Control (SATco):

- This tool is designed to assess postural trunk (your child's upper body) control.

- Your child will be asked to move with and without assistance (at different parts of their upper body)

\section{Pediatric Reach Test:}

- This test measures dynamic balance in the sitting and standing position

- The measurements will record how well your child can hold a sitting and standing position and how far the they can reach outside of their base of support in these positions.

- Your child will be asked to reach forward, to the left, and to the right.

Walking Assessments:

2 Minute Walk Test:

- This test measures your child's endurance while walking

- Your child will not be asked to do this test unless your child can walk without help from anyone. Your child can use a cane or walker for help.

- The goal is cover as much ground as possible in 2 minutes.

10 Meter Walk Test:

This test is designed to measure the speed that your child typically uses to walk. This test will not be performed if physical assistance from the therapist is required to walk. However, devices used to aid walking are allowed. Your child must be able to walk 10 meters in order to complete the test. The goal

is to walk the designated track as fast as safely possible. The time to walk over 10 meters is recorded.

\section{Spine and Hip Assessment}

\section{Scoliosis Assessment}

- Your child typically has an x-ray each year to check that your child's spine is growing straight. We will ask that copies of the $\mathrm{x}$-rays be sent to us and performed by our protocol and that your child approve sharing that information with us. 
- Your child typically has an $x$-ray to assess the position of their legs and hips as they grow. We will ask that copies of the $x$-rays be sent to us and performed by our protocol and that your child approve sharing that information with us.

\section{Quality of Life and Participation}

Quality of Life and Participation Questionnaires: We will ask you and your child to complete several questionnaires to help us better understand the impact of your child's injury and therapy on everyday life and activities. In addition we want to understand more about how your child feels from happy to sad to worried. It is possible that you and your child will be videotaped or an audio recording will be taken during this.

\section{Healthcare Utilization:}

We will ask your child to share information with us regarding your child's healthcare and associated cost of care. We will ask for information in regards to medications your child are taking, current or past surgeries, emergency room visits, hospital admissions, urinary tract infections, other occurrences requiring medical care, purchase or use of new assistive equipment, and current or past therapy regimens. It is possible that you and your child will be videotaped or an audio recording will be taken during this.

\section{Potential Risks}

The study may involve the following physical risks and/or discomforts:

Clinical Assessments

- Muscle Soreness (Rare $<1 \%$ )

- Changes in blood pressure, falls, fatigue (Less Likely 1-39\%) Scoliosis Assessment

- Muscle Soreness (Rare <1\%) Hip Dysplasia Assessment

- Muscle Soreness (Rare < $1 \%$ )

While you and your child are answering questions or participating in interviews, you or your child may feel some distress. You nor your child have to answer any questions that make youfeel uncomfortable.

In addition, your child may suffer harms that we have not seen before. This study may involve risks that are currently unforeseeable.

\section{Possible Pregnancy Risks}

There are no known animal or human data on the potential for birth defects.

\section{Benefits}

The information collected may not benefit your child directly; however, the information learned in this study may be helpful to others.

\section{Alternatives}

Instead of taking part in this study, you could choose to not participate. This will have no influence on your child's access and availability to routine care and evaluations. 
If your child is injured by being in this research study, the study doctor will arrange for your child to get medical treatment. The sponsor(s), study site nor your child's study doctor has set aside money to pay for treatment of any injury. You and your child's insurance (if applicable) will be billed for the treatment of these injuries. Before you agree to take part in this research study you should find out whether your child's insurance will cover an injury in this kind of research. You should talk to the study doctor or staff about this. If your child is injured, there is no money set aside for lost wages, discomfort, disability, etc. You do not give up you or your child's legal rights by signing this form. If you think your child has a research related injury, please call the study doctor, Andrea Behrman at 502-582-8695.

\section{Compensation}

Occasionally there are funds available to compensate participants for travel. If those funds are available, Dr. Behrman will make you aware prior to giving you this consent form. If you are compensated for travel, you will be paid based on the current federal mileage reimbursement rate. Because you will be paid to be in this study the University of Louisville must collect your name, address, social security number, ask you to sign a W-9 form, and keep records of how much you are paid. You may or may not be sent a Form 1099 by the University. This will only happen if you are paid $\$ 600$ or more in one year by the University. We are required by the Internal Revenue Service to collect this information and you may need to report the payment as income on your taxes. This information will be protected and kept secure in the same way that we protect your other private information. If you don't agree to give us this information, we cannot pay you for being in this study. You can still be in the study even if you don't want to be paid.

\section{Costs}

Unless your child is injured, there will not be additional costs to participate in this study.

\section{HIPAA Research Authorization}

The Health Insurance Portability and Accountability Act of 1996 (HIPAA) provides federal safeguards for protected health information $(\mathrm{PHI})$. Examples of $\mathrm{PHI}$ are your name, address, and birth date. $\mathrm{PHI}$ may also include your child's medical history, results of health exams and lab tests, drugs taken and results of this research study. Your child's PHI cannot be used or shared without your agreement, unless it meets one of the HIPAA exceptions.

State and federal privacy laws protect your health information. In most cases, health information that identifies your child can be used or shared by the research team only if you give your permission by signing this form.

If you sign this form your child's health information will be used and shared to answer the research questions described above and to make sure that the research was done correctly. The time period when information can be used or shared ends when all activities related to this study are completed.

Your access to your child's health information will not be limited during this study.

You do not have to sign this form. If you do not sign this form your child may not participate in the study and health information that identifies your child will not be shared with the research team.

Site(s) where health information about your child will be used or shared for this research: 
In our research, the research team will look at and may share information about your child and your child's health. Federal law requires that health care providers and researchers protect the privacy and security of health information that identifies your child. We may ask for your child's health information from the following:

\section{Affiliated Sites:}

University of Louisville

Jewish Hospital \& St. Mary’s Healthcare (Kentucky One Health)

\section{Protected health information (PHI) that will be used or shared for research}

Complete past medical history to determine eligibility criteria

History and physical exams

Laboratory, x-ray, and other tests

Photos, videotapes, or digital or other images

\section{Revocation of Research Authorization}

You may cancel the permission you have given to use and share your child's protected health information at any time. This means your child can tell us to stop using and sharing your child's protected health information. If you cancel your permission:

- We will stop collecting information about you and your child

- You may not withdraw information that we had before you told us to stop.

- We may already have used it or shared it.

- We may need it to complete the research.

To cancel your permission, you must complete a written "Revocation of Research Authorization" form located at the end of this document. You may also obtain a copy from your child's study doctor, designated personnel or from the Human Subjects Protections Program Office website (http://louisville.edu/research/humansubjects/subject-information)

\section{Confidentiality}

Total privacy cannot be guaranteed. We will protect your privacy to the extent permitted by law. If the results from this study are published, your child's name will not be made public. The following may look at your child's research and medical records:

- The funding agencies, Kosair Charities and Helmsley Foundation, and others hired by this agency to oversee the research

- The University of Louisville Institutional Review Board, Human Subjects Protection Program Office, Privacy Office and others involved in research administration at the University

- People who are responsible for research and HIPAA oversight at the institutions where the research is conducted

- Government agencies, such as:

- Office for Human Research Protections,

- Office of Civil Rights

- People responsible for billing, sending and receiving payments related to your participation in the study.

\section{Security}

You and your child's data will be kept private by assigning each of you a coded identification number. All research related files will be kept in a locked cabinet. The only people who will know that your child is a research subject are members of the research team and, if appropriate your child's physicians and nurses. No

Version Date 07.12.16 
information about you or your child, or provided by you or your child during the research, will be disclosed to others without your written permission, except if necessary to protect you and your child's rights or welfare (for example, if your child is injured and need emergency care), or if required by law.

When the results of the research are published or discussed in conferences, no information will be included that will reveal your child's identity.

The video recorded during the training sessions will be used for teaching and research purposes only. Such teaching and research purposes can include lectures, presentations, conferences, seminars, critiques of experimental procedures, and data analysis. Your child's identity will not be disclosed, as neither your child's name nor face will be shown. You agree that the tapes shall be kept for these purposes for an indefinite time

\section{Voluntary Participation}

Taking part in this study is completely voluntary. You may choose not to take part at all. If you decide not to be in this study even after your child has started the study, your child won't be penalized or lose any benefits for which your child qualifies. You and your child will be told about any new information learned during the study that could affect your decision to continue in the study.

\section{Termination}

The study doctor or the study sponsor has the right to stop this study at any point. The study doctor may take your child out of this study with or without your okay.

\section{Participation in Other Research Studies}

Your child may take part in this study if you or your child is currently in another research study. It is important to let the doctor know if you are in another research study.

\section{Contact Persons}

If you have any questions, concerns, or complaints about the research study, please contact Dr. Behrman at (502) $581-8675$ or other research team members at (502) $581-8695$. You will also be provided numbers to the research staff once enrolled in the study (e.g. personal cell phone numbers).

\section{Research Subject's Rights}

If you have any questions about you or your child's rights as a research subject, you may call the Human Subjects Protection Program Office at (502) 852-5188. You can discuss any questions about your child's rights as a research subject, in private, with a member of the Institutional Review Board (IRB). You may also call this number if you have other questions about the research, and you cannot reach the study doctor, or want to talk to someone else. The IRB is an independent committee made up of people from the University community, staff of the institutions, as well as people from the community not connected with these institutions. The IRB has reviewed this research study.

\section{Concerns and Complaints}

If you have concerns or complaints about the research or research staff and you do not wish to give your child's name, you may call 1-877-852-1167. This is a 24 hour hot line answered by people who do not work at the University of Louisville.

Version Date 07.12.16 
This informed consent document is not a contract. This document tells you what will happen during the study if you choose to take part. Your signature indicates that this study has been explained to you, that your questions have been answered, and that you agree to take part in the study. You are not giving up any legal rights to which you or your child are entitled by signing this informed consent document. You will be given a copy of this consent form to keep for your records.

\begin{tabular}{llr}
\hline Subject Name (Please Print) & Signature of Subject & Date Signed \\
\hline Printed Name of Legal Representative & Signature of Legal Representative & Date Signed
\end{tabular}
(if applicable)

$\overline{\text { Relationship of Legal Representative to Subject }}$

Printed Name of Person Explaining Consent $\bar{F}$ Form Signature of Person Explaining Date Signed (if other than the Investigator) Consent Form

\begin{tabular}{lll}
\hline Printed Name of Investigator & Signature of Investigator & Date Signed
\end{tabular}

\begin{tabular}{ll}
\hline List of Investigators: & Phone Numbers: \\
Andrea L. Behrman, PhD, PT & $502-581-8675$ \\
Karen Bertocci, PhD & $502-852-0279$ \\
Jennifer Thompson, MD & $502-588-3440$ \\
Dena Howland, PhD & $502-581-8675$
\end{tabular}


Advancing a New Trajectory of Outcomes for Children with Paralysis through Activity-Based Rehabilitation

Advancing a New Trajectory of Outco
Andrea L. Behrman PhD, PT, FAPTA

REVOCATION OF AUTHORIZATION FOR USE AND DISCLOSURE OF YOUR HEALTH INFORMATION

FOR RESEARCH

PI Address:220 Abraham Flexner Way, Suite 1521 Louisville.

KY 40202

Institutional Review Board

PI Phone: $502-581-8675$

OR

MedCenter One, Suite 200

$501 \mathrm{E}$. Broadway

Louisville. KY 40202

Do not sign this letter unless your child are withdrawing from this research. Your child will be sent confirmation that this notice was received.

To Whom It May Concern

I would like to discontinue my participation in the research study noted above. I understand that health information already collected will continue to be used as discussed in the Authorization I signed when joining the study.

Your options are (choose one):

\section{$\square$ Withdraw from Study \& Discontinue Authorization:}

Discontinue my authorization for the future use and disclosure of protected health information. In some instances, the research team may need to use your information even after you discontinue your authorization, for example, to notify you or government agencies of any health or safety concerns that were identified as part of your study participation

\section{$\square$ Withdraw from Study, but Continue Authorization:}

Allow the research team to continue collecting information from my personal health information. This would be done only as needed to support the goals of the study and would not be used for purposes other than those already described in the research authorization.

Printed Name and Signature of Subject

Signature of Subject's Legal Representative (if subject is unable to sign)

Printed Name of Subject's Legal Representative

Relationship of Legal Representative to Subject

Subject's Address

Optional:

I am ending my participation in this study because

\section{Date Signed}

Date Signed

Birthdate of Subject

Subject's Phone Number 


\section{Copyrights}

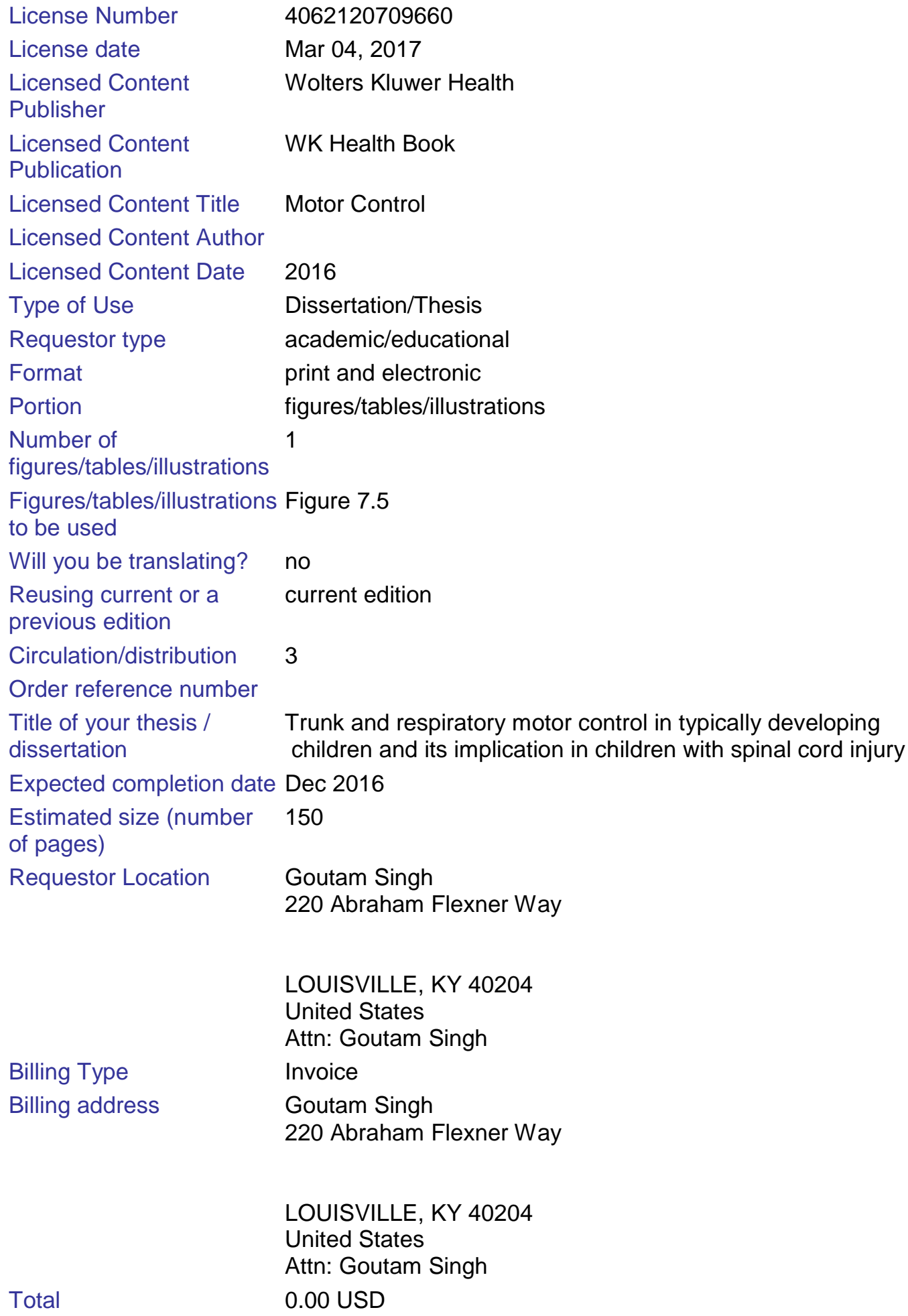

Total $\quad 0.00$ USD 


\section{CURRICULUM VITA \\ GOUTAM SINGH \\ 220 Abraham Flexner Way, $15^{\text {th }}$ floor, Louisville \\ g0sing06@cardmail.louisville.edu \\ 502-295-6440}

\section{EDUCATION}

PhD in Physiology

University of Louisville; Louisville, KY.

Master of Science in Exercise Physiology,

University of Louisville; Louisville, KY.

Dec 2012

Bachelor of Science in Physical Therapy

KIPT; Belgaum, India.

Dec 2008

\section{WORK EXPERIENCE}

Post-Doctoral Associate

Mar 2017- Current

Dept. of Neurological Surgery, University of Louisville,

Louisville, KY.

\section{Adjunct Instructor,}

Galen Nursing College, Louisville, KY.

Anatomy and Physiology-I

Graduate Research Assistant

Oct 2014-Feb 2017

Dept. Physiology, University of Louisville, Louisville, KY.

Graduate Teaching Assistant,

Department of Exercise Physiology,

University of Louisville, KY.

Physical Therapist

KLE'S Hospital \& MRC, Dr. Prabhakar Kore, Belgaum, India.

Physical Therapist

Vijaya Hospital; Belgaum, India
Aug 2009 - Jun 2010

Dec 2008-Jun 2009 


\section{RESEARCH PROJECT}

Dissertation Research (2014-2017): "Neurophysiological mechanisms of respiratory and trunk motor control in typically developing children and its correlation to children with spinal cord injury".

Mentor: Dr. Alexander Ovechkin, Department of Neurological surgery, Louisville, KY.

Thesis research (2010-2012) "Effects of 3. \% menthol gel on knee extensor muscle strength and perceived pain among college athletes". Mentor: Dr. Saori Martin. Department of Exercise Physiology, University of Louisville; Louisville, KY.

\section{FELLOWSHIPS:}

Graduate fellowship award by College of Education and Human Development2011-2012.

Integrated Program in Biomedical Science Fellowship-2012-2014.

\section{PROFESSIONAL MEMBERSHIP}

Society for Neuroscience

American Physiology Association

\section{AWARDS AND ACCOMPLISHMENT}

IPIBS Fellowship award-2012-2014

International Student Tuition Support Scholarship-2012

Graduate citation award 2012-University of Louisville-2012

Distinction award for securing $1^{\text {st }}$ place at KIPT Belguam-2007

\section{MANUSCRIPT IN PREPERATION}

1. Respiratory motor deficit in children with spinal cord injury (April 2017).

2. Development of trunk motor control in neurologically intact children and its correlation to

children with spinal cord injury (May 2017).

3. Validation of Segmental assessment of trunk control (SATCo) in children with spinal cord injury (May 2017).

\section{POSTER AND ORAL PRESENTATION}

1. Goutam Singh, Shelly Trimble, Andrea Behrman, Sevda Aslan, Alexander Ovechkin. Respiratory motor deficit in children with spinal cord injury. Society for Neuroscience, San Diego, Nov 2016.

2. Goutam Singh, Shelly Trimble, Andrea Behrman, Sevda Aslan, Alexander Ovechkin. Development of trunk \& respiratory motor control in children with spinal cord injury. National Neurotrauma Society, Lexington, June 2016.

3. Goutam Singh, Shelly Trimble, Andrea Behrman, Sevda Aslan, Alexander Ovechkin. Evaluation of respiratory and trunk motor control in typically developing children and its implication in children with spinal cord injury at Department of Physiology and Biophysics, May 2015. 
4. Goutam Singh, Shelly Trimble, Andrea Behrman, Sevda Aslan, Alexander Ovechkin. Respiratory motor control assessment in neurologically intact children and its correlation to children with spinal cord injury. Research Louisville, Louisville, Oct 2015.

5. Goutam Singh, Ann Swank, Saori Hanaki Martin. Effects of $3.5 \%$ menthol gel on knee extensor muscle strength and perceived pain among college athletes. American collage of sports medicine (ACSM). Indiana, May 2013.

\section{PROFESSIONAL WORK AND OUTREACH}

Graduate Research Assistant, Frazier Rehab Center

Aug 2014-Present

University of Louisville, Louisville, KY

Physical Therapist

Aug 2009 - Jun 2010

KLE'S Hospital \& MRC, Dr. Prabhakar Kore; Belgaum, India

Physical Therapist

Dec 2008-Jun 2009

Vijaya Hospital; Belgaum, India

Student Academic Grievance Committee Member, June 2015-Present

University of Louisville; Louisville, KY

Graduate Teaching Assistant, Department of Exercise Physiology,

Jan 2012-May 2012

University of Louisville; Louisville, KY

Student Health Advocate, Campus Health Services

Aug 2011-Dec 2011

University of Louisville; Louisville, KY

Student Ambassador, Exercise Physiology Program

Jan 2011-Aug 2011

University of Louisville; Louisville, KY

Student Coordinator, Cultural Center

Sep 2011-Dec 2011

University of Louisville: Louisville, KY

Student Leader, International Outreach Diversity Program

Oct 2010 - May 2011

University of Louisville; Louisville, KY

\section{CORE QUALIFICATIONS}

- Strong ability to assess physical functions.

- Pulmonary function testing in children and adults.

- Substantial experience in targeted fitness and training guidance.

- Excellent interactive and communication skills.

- Motion Analysis (Cortex), MATLAB, HSCIP, LABVIEW, LABCHART data acquisition \& processing.

- R-Studio statistics.

- sEMG data acquisition and analysis. 


\section{WORKSHOPS AND SYPMOSIA}

Pediatric Care Symposium 2017

Locomotor Training-Pediatric Neurorecovery Training Institute2015

Workshop on CARDIAC REHABILITATION 2008

Workshop on Pediatric resuscitation 2008 